\title{
Approximating Constraint Satisfaction Problems on High-Dimensional Expanders
}

\author{
Vedat Levi Alev * $\quad$ Fernando Granha Jeronimo $^{\dagger} \quad$ Madhur Tulsiani $^{\ddagger}$
}

We consider the problem of approximately solving constraint satisfaction problems with arity $k>2$ ( $k$-CSPs) on instances satisfying certain expansion properties, when viewed as hypergraphs. Random instances of $k$-CSPs, which are also highly expanding, are wellknown to be hard to approximate using known algorithmic techniques (and are widely believed to be hard to approximate in polynomial time). However, we show that this is not necessarily the case for instances where the hypergraph is a high-dimensional expander.

We consider the spectral definition of high-dimensional expansion used by Dinur and Kaufman [FOCS 2017] to construct certain primitives related to PCPs. They measure the expansion in terms of a parameter $\gamma$ which is the analogue of the second singular value for expanding graphs. Extending the results by Barak, Raghavendra and Steurer [FOCS 2011] for 2-CSPs, we show that if an instance of MAX k-CSP over alphabet $[q]$ is a highdimensional expander with parameter $\gamma$, then it is possible to approximate the maximum fraction of satisfiable constraints up to an additive error $\varepsilon$ using $q^{O(k)} \cdot(k / \varepsilon)^{O(1)}$ levels of the sum-of-squares SDP hierarchy, provided $\gamma \leq \varepsilon^{O(1)} \cdot(1 /(k q))^{O(k)}$.

Based on our analysis, we also suggest a notion of threshold-rank for hypergraphs, which can be used to extend the results for approximating 2-CSPs on low threshold-rank graphs. We show that if an instance of MAX k-CSP has threshold rank $r$ for a threshold $\tau=(\varepsilon / k)^{O(1)} \cdot(1 / q)^{O(k)}$, then it is possible to approximately solve the instance up to additive error $\varepsilon$, using $r \cdot q^{O(k)} \cdot(k / \varepsilon)^{O(1)}$ levels of the sum-of-squares hierarchy. As in the case of graphs, high-dimensional expanders (with sufficiently small $\gamma$ ) have threshold rank 1 according to our definition.

\footnotetext{
*Supported by NSERC Discovery Grant 2950-120715, NSERC Accelerator Supplement 2950-120719, and partially supported by NSF awards CCF-1254044 and CCF-1718820. vlalev@uwaterloo.ca.

†Supported in part by NSF grants CCF-1254044 and CCF-1816372. granha@uchicago.edu.

$\ddagger$ Supported by NSF grants CCF-1254044 and CCF-1816372. madhurt@ttic. edu
} 


\section{Contents}

1 Introduction 1

2 Preliminaries and Notation 4

2.1 Linear Algebra . . . . . . . . . . . . . . . . . . . . . . . 4

2.2 High-Dimensional Expanders . . . . . . . . . . . . . . . . 4

2.2.1 Simplicial Complexes . . . . . . . . . . . . . . . . 4

2.2.2 Walks and Measures on Simplicial Complexes . . . . . . . . . . 5

2.2.3 High-Dimensional Expansion . . . . . . . . . . . . . 6

2.3 Constraint Satisfaction Problems (CSPs) . . . . . . . . . . . . . . 6

2.4 Sum-of-Squares Relaxations and $t$-local PSD Ensembles . . . . . . . . . . . 7

3 Proof Overview: Approximating MAX 4-XOR $\quad 8$

4 Walks $\quad 11$

4.1 The Canonical and the Swap Walks on a Simplicial Complex . . . . . . . . 13

4.2 Swap Walks are Height Independent . . . . . . . . . . . . . . . . . . 15

4.3 Canonical Walks in Terms of the Swap Walks . . . . . . . . . . . . . . 16

4.4 Inversion: Swap Walks in Terms of Canonical Walks . . . . . . . . . . . . 17

5 Spectral Analysis of Swap Walks $\quad 18$

5.1 Square Swap Walks $S_{k, k} \ldots \ldots \ldots$. . . . . . . . . . . . . 19

5.2 Expanding Posets and Balanced Operators . . . . . . . . . . . . . . . 21

5.3 Quadratic Forms over Balanced Operators . . . . . . . . . . . . . . 23

5.4 Rectangular Swap Walks $S_{k, l} \ldots \ldots \ldots \ldots$. . . . . . . . . . . . . . . . . . . . . . . .

5.5 Bipartite Kneser Graphs - Complete Complex . . . . . . . . . . . . . . . . . . 32

6 Approximating Max- $k$-CSP $\quad 34$

6.1 Breaking Correlations for Expanding CSPs: Proof of Theorem 6.3 . . . . . 38

6.2 The Glorified Triangle Inequality: Proof of Lemma 6.6 . . . . . . . . . . . . . 41

7 High-Dimensional Threshold Rank 43

7.1 Breaking Correlations for Splittable CSPs: Proof of Theorem 7.3 . . . . . . . 44

8 Quantum k-local Hamiltonian 46

A From Local to Global Correlation $\quad 52$

B Harmonic Analysis on HDXs 56 


\section{Introduction}

We consider the problem of approximately solving constraint satisfaction problems (CSPs) on instances satisfying certain expansion properties. The role of expansion in understanding the approximability of CSPs with two variables in each constraint (2-CSPs) has been extensively studied and has led to several results, which can also be viewed as no-go results for PCP constructions (since PCPs are hard instances of CSPs). It was shown by Arora et al. $\left[\mathrm{AKK}^{+}\right.$08] (and strengthened by Makarychev and Makarychev [MM11]) that the Unique Games problem is easily approximable on expanding instances, thus proving that the Unique Games Conjecture of Khot [Kho02] cannot be true for expanding instances. Their results were extended to all 2-CSPs and several partitioning problems in works by Barak, Raghavendra and Steurer [BRS11], Guruswami and Sinop [GS11], and Oveis Gharan and Trevisan [OGT15] under much weaker notions of expansion.

We consider the following question:

When are expanding instances of $k$-CSPs easy for $k>2$ ?

At first glance, the question does not make much sense, since random instances of $k$-CSPs (which are also highly expanding) are known to be hard for various models of computation (see [KMOW17] for an excellent survey). However, while the kind of expansion exhibited by random instances of CSPs is useful for constructing codes, it is not sufficient for constructing primitives for PCPs, such as locally testable codes [BSHR05]. On the other hand, objects such as high-dimensional expanders, which possess a form of "structured multi-scale expansion" have been useful in constructing derandomized direct-product and direct-sum tests (which can be viewed as locally testable distance amplification codes) [DK17], lattices with large distance [KM18], list-decodable direct product codes [DHK $\left.{ }^{+} 18\right]$, and are thought to be intimately connected with PCPs [DK17]. Thus, from the PCP perspective, it is more relevant to ask if this form of expansion can be used to efficiently approximate constraint satisfaction problems.

Connections to coding theory. Algorithmic results related to expanding CSPs are also relevant for the problem of decoding locally testable codes. Consider a code $C$ constructed via $k$-local operations (such as $k$-fold direct-sum) on a base code $C_{0}$ with smaller distance. Then, a codeword in $C$ is simply an instance of a CSP, where each bit places a constraint on $k$ bits (which is $k$-XOR in case of direct sum) of the relevant codeword in $C_{0}$. The task of decoding a noisy codeword is then equivalent to finding an assignment in $C_{0}$, satisfying the maximum number of constraints for the above instance. Thus, algorithms for solving CSPs on expanding instances may lead to new decoding algorithms for codes obtained by applying local operations to a base code. In fact, the list decoding algorithm for direct-product codes by Dinur et al. [DHK $\left.{ }^{+} 18\right]$ also relied on algorithmic results for expanding unique games. Since all constructions of locally testable codes need to have at least some weak expansion [DK12], it is interesting to understand what notions of expansion are amenable to algorithmic techniques.

High-dimensional expanders and our results. A $d$-dimensional expander is a downwardclosed hypergraph (simplicial complex), say $X$, with edges of size at most $d+1$, such that for every hyperedge $\mathfrak{a} \in X$ (with $|\mathfrak{a}| \leq d-1$ ), a certain "neighborhood graph" $G\left(X_{\mathfrak{a}}\right)$ is a 
spectral expander ${ }^{1}$. Here, the graph $G\left(X_{\mathfrak{a}}\right)$ is defined to have the vertex $\operatorname{set}\{i \mid \mathfrak{a} \cup\{i\} \in X\}$ and edge-set $\{i, j \mid \mathfrak{a} \cup\{i, j\} \in X\}$. If the (normalized) second singular value of each of the neighborhood graphs is bounded by $\gamma, X$ is said to be a $\gamma$-high-dimensional expander $(\gamma$-HDX).

Note that (the downward closure of) a random sparse $(d+1)$-uniform hypergraph, say with $n$ vertices and $c \cdot n$ edges, is very unlikely to be a $d$-dimensional expander. With high probability, no two hyperedges share more than one vertex and thus for any $i \in$ $[n]$, the neighborhood graph $G_{i}$ is simply a disjoint union of cliques of size $d$, which is very far from an expander. While random hypergraphs do not yield high-dimensional expanders, such objects are indeed known to exists via (surprising) algebraic constructions [LSV05b, LSV05a, KO18a, CTZ18] and are known to have several interesting properties and applications [KKL16, DHK ${ }^{+} 18, \mathrm{KM} 17, \mathrm{KO} 18 b, \mathrm{DDFH18,} \mathrm{DK17,} \mathrm{PRT16].}$

Expander graphs can simply be thought of as the one-dimensional case of the above definition. The results of Barak, Raghavendra and Steurer [BRS11] for 2-CSPs yield that if the constraint graph of a 2-CSP instance (with size $n$ and alphabet size $q$ ) is a sufficiently good (one dimensional) spectral expander, then one can efficiently find solutions satisfying OPT $-\varepsilon$ fraction of constraints, where OPT denotes the maximum fraction of constraints satisfiable by any assignment. Their algorithm is based on $(q / \varepsilon)^{O(1)}$ levels of the Sumof-Squares (SoS) SDP hierarchy, and the expansion requirement on the constraint graph is that the (normalized) second singular value should be at most $(\varepsilon / q)^{O(1)}$. We show a similar result for $k$-CSPs when the corresponding simplicial complex $X_{\mathfrak{J}}$, which is obtained by including one hyperedge for each constraint and taking a downward closure, is a sufficiently good $(k-1)$-dimensional expander.

Theorem 1.1 (Informal). Let $\mathfrak{I}$ be an instance of MAX k-CSP on $n$ variables taking values over an alphabet of size $q$, and let $\varepsilon>0$. Let the simplicial complex $X_{\mathfrak{I}}$ be a $\gamma-H D X$ with $\gamma=\varepsilon^{O(1)}$. $(1 /(k q))^{O(k)}$. Then, there is an algorithm based on $(k / \varepsilon)^{O(1)} \cdot q^{O(k)}$ levels of the Sum-of-Squares hierarchy, which produces an assignment satisfying OPT $-\varepsilon$ fraction of the constraints.

Remark 1.2. While the level-t relaxation for MAX k-CSP can be solved in time $(n q)^{O(t)}$ [RW17], the rounding algorithms used by [BRS11] and our work do not need the full power of this relaxation. Instead, they are captured by the "local rounding" framework of Guruswami and Sinop [GS12] who show how to implement a local rounding algorithm based on t levels of the SoS hierarchy, in time $q^{O(t)} \cdot n^{O(1)}$ (where $q$ denotes the alphabet size).

Our techniques. We start by using essentially the same argument for analyzing the SoS hierarchy as was used by [BRS11] (specialized to the case of expanders). They viewed the SoS solution as giving a joint distribution on each pair of variables forming a constraint, and proved that for sufficiently expanding graphs, these distributions can be made close to product distributions, by conditioning on a small number of variables (which governs the number of levels required). Similarly, we consider the conditions under which joint distributions on $k$-tuples corresponding to constraints can be made close to product distributions. Since the [BRS11] argument shows how to split a joint distribution into two marginals, we can use it to recursively split a set of size $k$ into two smaller ones (one can think of all splitting operations as forming a binary tree with $k$ leaves).

\footnotetext{
${ }^{1}$ While there are several definitions of high-dimensional expanders, we consider the one by Dinur and Kaufman [DK17], which is most closely related to spectral expansion, and was also the one shown to be related to PCP applications. Our results also work for a weaker but more technical definition by Dikstein et al. [DDFH18], which we defer till later.
} 
However, our arguments differ in the kind of expansion required to perform the above splitting operations. In the case of the 2-CSP, one splits along the edges of the constraint graph, and thus we only need the expansion of the contraint graph (which is part of the assumption). However, in the case of $k$-CSPs, we may split a set of size $\left(\ell_{1}+\ell_{2}\right)$ into disjoint sets of size $\ell_{1}$ and $\ell_{2}$. This requires understanding the expansion of the following family of (weighted) bipartite graphs arising from the complex $X_{\mathfrak{I}}$ : The vertices in the graph are sets of variables of size $\ell_{1}$ and $\ell_{2}$ that occur in some constraint, and the weight of an edge $\left\{\mathfrak{a}_{1}, \mathfrak{a}_{2}\right\}$ for $\mathfrak{a}_{1} \cap \mathfrak{a}_{2}=\varnothing$, is proportional to the probability that a random constraint contains $\mathfrak{a}_{1} \sqcup \mathfrak{a}_{2}$. Note that this graph may be weighted even if the $k$-CSP instance $\mathfrak{I}$ is unweighted.

We view the above graphs as random walks, which we call "swap walks" on the hyperedges (faces) in the complex $X$. While several random walks on high-dimensional expanders have been shown to have rapid mixing [KM17, KO18b, DK17, LLP17], we need a stronger condition. To apply the argument from [BRS11], we not only need that the second singular value is bounded away from one, but require it to be an arbitrarily small constant (as a function of $\varepsilon, k$ and $q$ ). We show that this is indeed ensured by the condition that $\mathfrak{a}_{1} \cap \mathfrak{a}_{2}=\varnothing$, and obtain a bound of $k^{O(k)} \cdot \gamma$ on the second singular value. This bound, which constitutes much of the technical work in the paper, is obtained by first expressing these walks in terms of more canonical walks, and then using the beautiful machinery of harmonic analysis on expanding posets by Dikstein et al. [DDFH18] to understand their spectra.

The swap walks analyzed above represent natural random walks on simplicial complexes, and their properties may be of independent interest for other applications. Just as the high-dimensional expanders are viewed as "derandomized" versions of the complete complex (containing all sets of size at most $k$ ), one can view the swap walks as derandomized versions of (bipartite) Kneser graphs, which have vertex sets $\left(\begin{array}{c}{[n]} \\ \ell_{1}\end{array}\right)$ and $\left(\begin{array}{c}{[n]} \\ \ell_{2}\end{array}\right)$, and edges $(\mathfrak{a}, \mathfrak{b})$ iff $\mathfrak{a} \cap \mathfrak{b}=\varnothing$. We provide a more detailed and technical overview in Section 3 after discussing the relevant preliminaries in Section 2.

High-dimensional threshold rank. The correlation breaking method in [BRS11] can be applied as long as the graph has low threshold rank i.e., the number of singular values above a threshold $\tau=(\varepsilon / q)^{O(1)}$ is bounded. Similarly, the analysis described above can be applied, as long as all the swap walks which arise when splitting the $k$-tuples have bounded threshold rank. This suggests a notion of high-dimensional threshold rank for hypergraphs (discussed in Section 7), which can be defined in terms of the threshold ranks of the relevant swap walks. We remark that it is easy to show that dense hypergraphs (with $\Omega\left(n^{k}\right)$ hyperedges) have small-threshold rank according to this notion, and thus it can be used to recover known algorithms for approximating $k$-CSPs on dense instances [FK96] (as was true for threshold rank in graphs).

Other related work. While we extend the approach taken by [BRS11] for 2-CSPs, somewhat different approaches were considered by Guruswami and Sinop [GS11], and OveisGharan and Trevisan [OGT15]. The work by Guruswami and Sinop relied on the expansion of the label extended graph, and used an analysis based on low-dimensional approximations of the SDP solution. Oveis-Gharan and Trevisan used low-threshold rank assumptions to obtain a regularity lemma, which was then used to approximate the CSP. For the case of $k$-CSPs, the Sherali-Adams hierarchy can be used to solve instances with bounded 
treewidth [WJ04] and approximately dense instances [YZ14, MR17]. Brandao and Harrow [BH13] also extended the results by [BRS11] for 2-CSPs to the case of 2-local Hamiltonians. We show that their ideas can also be used to prove a similar extension of our results to $k$-local Hamiltonians on high-dimensional expanders.

In case of high-dimensional expanders, in addition to canonical walks described here, a "non-lazy" version of these walks (moving from $\mathfrak{s}$ to $\mathfrak{t}$ only if $\mathfrak{s} \neq \mathfrak{t}$ ) was also considered by Kaufman and Oppenheim [KO18b], Anari et al. [ALGV18] and Dikstein et al. [DDFH18]. The swap walks studied in this paper were also considered independently in a very recent work of Dikstein and Dinur [DD19] (under the name "complement walks").

In a recent follow-up work $\left[\mathrm{AJQ}^{+} 19\right]$, the algorithms developed here were also used to obtain new unique and list decoding algorithms for direct sum and direct product codes, obtained by a "lifting" a base code $C_{0}$ via $k$-local operations to amplify distance. This work also showed that the hypergraphs obtained by considering collections of length- $k$ walks on an expanding graph also satisfy (a slight variant of) splittability, and admit similar algorithms.

\section{Preliminaries and Notation}

\subsection{Linear Algebra}

Recall that for an operator $\mathrm{A}: V \rightarrow W$ between two finite-dimensional inner product spaces $V$ and $W$, the operator norm can be written as

$$
\|\mathrm{A}\|_{\text {op }}=\sup _{f, g \neq 0} \frac{\langle\mathrm{A} f, g\rangle}{\|f\|\|g\|} .
$$

Also, for such an $\mathrm{A}$ the adjoint $\mathrm{A}^{\dagger}: W \rightarrow V$ is defined as the (unique) operator satisfying $\langle\mathrm{A} f, g\rangle=\left\langle f, \mathrm{~A}^{\dagger} g\right\rangle$ for all $f \in V, g \in W$. For $\mathrm{A}: V \rightarrow W$, we take $\|\mathrm{A}\|_{\text {op }}=\sigma_{1}(\mathrm{~A}) \geq \sigma_{2}(\mathrm{~A}) \geq$ $\cdots \geq \sigma_{r}(\mathrm{~A})>0$ to be its singular values in descending order. Note that for $\mathrm{A}: V \rightarrow V$, $\sigma_{2}(\mathrm{~A})$ denotes its second largest eigenvalue in absolute value.

\subsection{High-Dimensional Expanders}

A high-dimensional expander (HDX) is a particular kind of downward-closed hypergraph (simplicial complex) satisfying an expansion requirement. We elaborate on these properties and define well known natural walks on HDXs below.

\subsubsection{Simplicial Complexes}

Definition 2.1. A simplicial complex $X$ with ground set $[n]$ is a downward-closed collection of subsets of $[n]$ i.e., for all sets $\mathfrak{s} \in X$ and $\mathfrak{t} \subseteq \mathfrak{s}$, we also have $\mathfrak{t} \in X$. The sets in $X$ are also referred to as faces of $X$.

We use the notation $X(i)$ to denote the collection of all faces $\mathfrak{s}$ in $X$ with $|\mathfrak{s}|=i$. When faces are of cardinality at most $d$, we also use the notation $X(\leq d)$ to denote all the faces of $X$. By convention, we take $X(0):=\{\varnothing\}$. 
A simplicial complex $X(\leq d)$ is said to be a pure simplicial complex if every face of $X$ is contained in some face of size $d$. Note that in a pure simplicial complex $X(\leq d)$, the top slice $X(d)$ completely determines the complex.

Note that it is more common to associate a geometric representation to simplicial complexes, with faces of cardinality $i$ being referred to as faces of dimension $i-1$ (and the collection being denoted by $X(i-1)$ instead of $X(i))$. However, since we will only be treating these as hypergraphs, we prefer to index faces by their cardinality, to improve readability of related expressions.

An important simplicial complex is the complete complex.

Definition 2.2 (Complete Complex $\Delta_{d}(n)$ ). We denote by $\Delta_{d}(n)$ the complete complex with faces of size at most d i.e., $\Delta_{d}(n):=\{\mathfrak{s} \subseteq[n]|| \mathfrak{s} \mid \leq d\}$.

\subsubsection{Walks and Measures on Simplicial Complexes}

Let $C^{k}$ denote the space of real valued functions on $X(k)$ i.e.,

$$
C^{k}:=\{f \mid f: X(k) \rightarrow \mathbb{R}\} \cong \mathbb{R}^{X(k)} .
$$

We describe natural walks on simplicial complexes considered in [DK17, DDFH18, KO18b], as stochastic operators, which map functions in $C^{i}$ to $C^{i+1}$ and vice-versa.

To define the stochastic operators associated with the walks, we first need to describe a set of probability measures which serve as the stationary measures for these random walks. For a pure simplicial complex $X(\leq d)$, we define a collection of probability measures $\left(\Pi_{1}, \ldots \Pi_{d}\right)$, with $\Pi_{i}$ giving a distribution on faces in the slice $X(i)$.

Definition 2.3 (Probability measures $\left(\Pi_{1}, \ldots, \Pi_{d}\right)$ ). Let $X(\leq d)$ be a pure simplicial complex and let $\Pi_{d}$ be an arbitrary probability measure on $X(d)$. We define a coupled array of random variables $\left(\mathfrak{s}^{(d)}, \ldots, \mathfrak{s}^{(1)}\right)$ as follows: sample $\mathfrak{s}^{(d)} \sim \Pi_{d}$ and (recursively) for each $i \in[d]$, take $\mathfrak{s}^{(i-1)}$ to be a uniformly random subset of $\mathfrak{s}^{(i)}$, of size $i-1$.

The distributions $\Pi_{d-1}, \ldots, \Pi_{1}$ are then defined to be the marginal distributions of the random variables $\mathfrak{s}^{(d-1)}, \ldots, \mathfrak{s}^{(1)}$ as defined above.

The following is immediate from the definition above.

Proposition 2.4. Let $\mathfrak{a} \in X(\ell)$ be an arbitrary face. For all $j \geq 0$, one has

$$
\sum_{\substack{\mathfrak{b} \in X(\ell+j): \\
\mathfrak{b} \supseteq \mathfrak{a}}} \Pi_{\ell+j}(\mathfrak{b})=\left(\begin{array}{c}
\ell+j \\
j
\end{array}\right) \cdot \Pi_{\ell}(\mathfrak{a}) .
$$

For all $k$, we define the inner product of functions $f, g \in C^{k}$, according to associated measure $\Pi_{k}$

$$
\langle f, g\rangle=\underset{\mathfrak{s} \sim \Pi_{k}}{\mathbb{E}}[f(\mathfrak{s}) g(\mathfrak{s})]=\sum_{\mathfrak{s} \in X(k)} f(\mathfrak{s}) g(\mathfrak{s}) \cdot \Pi_{k}(\mathfrak{s}) .
$$

We now define the up and down operators $\mathrm{U}_{i}: C^{i} \rightarrow C^{i+1}$ and $\mathrm{D}_{i+1}: \mathrm{C}^{i+1} \rightarrow \mathrm{C}^{i}$ as

$$
\begin{aligned}
{\left[\mathrm{U}_{i} g\right](\mathfrak{s}) } & =\underset{\mathfrak{s}^{\prime} \in X(i), \mathfrak{s}^{\prime} \subseteq \mathfrak{s}}{\mathbb{E}}\left[g\left(\mathfrak{s}^{\prime}\right)\right]=\frac{1}{i+1} \cdot \sum_{x \in \mathfrak{s}} g(\mathfrak{s} \backslash\{x\}) \\
{\left[\mathrm{D}_{i+1} g\right](\mathfrak{s}) } & =\underset{\mathfrak{s}^{\prime} \sim \prod_{i+1} \mid \mathfrak{s}^{\prime} \supset \mathfrak{s}}{\mathbb{E}}\left[g\left(\mathfrak{s}^{\prime}\right)\right]=\frac{1}{i+1} \cdot \sum_{x \notin \mathfrak{s}} g(\mathfrak{s} \sqcup\{x\}) \cdot \frac{\Pi_{i+1}(\mathfrak{s} \sqcup\{x\})}{\Pi_{i}(\mathfrak{s})}
\end{aligned}
$$


An important consequence of the above definition is that $\mathrm{U}_{i}$ and $\mathrm{D}_{i+1}$ are adjoints with respect to the inner products of $C^{i}$ and $C^{i+1}$.

Fact 2.5. $\mathrm{U}_{i}=\mathrm{D}_{i+1}^{\dagger}$, i.e., $\left\langle\mathrm{U}_{i} f, g\right\rangle=\left\langle f, \mathrm{D}_{i+1} g\right\rangle$ for every $f \in C^{i}$ and $g \in C^{i+1}$.

Note that the operators can be thought of as defining random walks in a simplicial complex $X(\leq d)$. The operator $\mathrm{U}_{i}$ moves down from a face $\mathfrak{s} \in X(i+1)$ to a face $\mathfrak{s}^{\prime} \in X(i)$, but lifts a function $g \in C^{i}$ up to a function $U g \in C^{i+1}$. Similarly, the operator $\mathrm{D}_{i+1}$ can be thought of as defining a random walk which moves up from $\mathfrak{s} \in X(i)$ to $\mathfrak{s}^{\prime} \in X(i+1)$. It is easy to verify that these walks respectively map the measure $\Pi_{i+1}$ to $\Pi_{i}$, and $\Pi_{i}$ to $\Pi_{i+1}$.

\subsubsection{High-Dimensional Expansion}

We recall the notion of high-dimensional expansion (defined via local spectral expansion) considered by [DK17]. We first need a few pieces of notation.

For a complex $X(\leq d)$ and $\mathfrak{s} \in X(i)$ for some $i \in[d]$, we denote by $X_{\mathfrak{s}}$ the link complex

$$
X_{\mathfrak{s}}:=\{\mathfrak{t} \backslash \mathfrak{s} \mid \mathfrak{s} \subseteq \mathfrak{t} \in X\} .
$$

When $|\mathfrak{s}| \leq d-2$, we also associate a natural weighted graph $G\left(X_{\mathfrak{s}}\right)$ to a link $X_{\mathfrak{s}}$, with vertex set $X_{\mathfrak{s}}(1)$ and edge-set $X_{\mathfrak{s}}(2)$. The edge-weights are taken to be proportional to the measure $\Pi_{2}$ on the complex $X_{\mathfrak{s}}$, which is in turn proportional to the measure $\Pi_{|\mathfrak{s}|+2}$ on $X$. The graph $G\left(X_{\mathfrak{s}}\right)$ is referred to as the skeleton of $X_{\mathfrak{s}}$. Dinur and Kaufman [DK17] define high-dimensional expansion in terms of spectral expansion of the skeletons of the links.

Definition 2.6 ( $\gamma$-HDX from [DK17]). A simplicial complex $X(\leq d)$ is said to be $\gamma$-High Dimensional Expander $\left(\gamma\right.$-HDX) if for every $0 \leq i \leq d-2$ and for every $\mathfrak{s} \in X(i)$, the graph $G\left(X_{\mathfrak{s}}\right)$ satisfies $\sigma_{2}\left(G\left(X_{\mathfrak{s}}\right)\right) \leq \gamma$, where $\sigma_{2}\left(G\left(X_{\mathfrak{s}}\right)\right)$ denotes the second singular value of the (normalized) adjacency matrix of $G\left(X_{\mathfrak{s}}\right)$.

\subsection{Constraint Satisfaction Problems (CSPs)}

A $k$-CSP instance $\mathfrak{I}=(H, \mathcal{C}, w)$ with alphabet size $q$ consists of a $k$-uniform hypergraph, a set of constraints

$$
\mathcal{C}=\left\{\mathcal{C}_{\mathfrak{a}} \subseteq[q]^{\mathfrak{a}}: \mathfrak{a} \in H\right\}
$$

and a non-negative weight function $w \in \mathbb{R}_{+}^{H}$ on the constraints, satisfying $\sum_{\mathfrak{a} \in H} w(a)=1$.

A constraint $\mathcal{C}_{\mathfrak{a}}$ is said to be satisfied by an assignment $\eta$ if we have $\left.\eta\right|_{\mathfrak{a}} \in \mathcal{C}_{\mathfrak{a}}$ i.e., the restriction of $\eta$ on $\mathfrak{a}$ is contained in $\mathcal{C}_{\mathfrak{a}}$. We write, $\operatorname{SAT}_{\mathfrak{I}}(\eta)$ for the (weighted fraction of the constraints) satisfied by the assignment $\eta$ i.e.,

$$
\operatorname{SAT}_{\mathfrak{I}}(\eta)=\sum_{\mathfrak{a} \in H} w(\mathfrak{a}) \cdot \mathbf{1}\left[\left.\eta\right|_{\mathfrak{a}} \in \mathcal{C}_{\mathfrak{a}}\right]=\underset{\mathfrak{a} \sim w}{\mathbb{E}}\left[\mathbf{1}\left[\left.\eta\right|_{\mathfrak{a}} \in \mathcal{C}_{\mathfrak{a}}\right]\right]
$$

We denote by $\operatorname{OPT}(\mathfrak{I})$ the maximum of $\operatorname{SAT}_{\mathfrak{I}}(\eta)$ over all $\eta \in[q]^{V(H)}$.

Any $k$-uniform hypergraph $H$ can be associated with a pure simplicial complex in a canonical way by just setting $X_{\mathfrak{I}}=\{\mathfrak{b}: \exists \mathfrak{a} \in H$ and $\mathfrak{a} \supseteq \mathfrak{b}\}$ - notice that $X_{\mathfrak{I}}(k)=H$. We will refer to this complex as the constraint complex of the instance $\mathfrak{I}$. The probability distribution $\Pi_{k}$ on $X_{\mathfrak{I}}$ will be derived from the weights function $w$ of the constraint, i.e

$$
\Pi_{k}(\mathfrak{a})=w(\mathfrak{a}) \quad \forall \mathfrak{a} \in X_{\mathfrak{I}}(k)=H .
$$




\subsection{Sum-of-Squares Relaxations and $t$-local PSD Ensembles}

The Sum-of-Squares (SoS) hierarchy gives a sequence of increasingly tight semidefinite programming relaxations for several optimization problems, including CSPs. Since we will use relatively few facts about the SoS hierarchy, already developed in the analysis of Barak, Raghavendra and Steurer [BRS11], we will adapt their notation of $t$-local distributions to describe the relaxations. For a $k$-CSP instance $\mathfrak{I}=(H, \mathcal{C}, w)$ on $n$ variables, we consider the following semidefinite relaxation given by $t$-levels of the SoS hierarchy, with vectors $v_{(S, \alpha)}$ for all $S \subseteq[n]$ with $|S| \leq t$, and all $\alpha \in[q]^{S}$. Here, for $\alpha_{1} \in[q]^{S_{1}}$ and $\alpha_{2} \in[q]^{S_{2}}$, $\alpha_{1} \circ \alpha_{2} \in[q]^{S_{1} \cup S_{2}}$ denotes the partial assignment obtained by concatenating $\alpha_{1}$ and $\alpha_{2}$.

$\begin{array}{|rrr|}\text { maximize } \quad \underset{\mathfrak{a} \sim w}{\mathbb{E}}\left[\sum_{\alpha \in C_{\mathfrak{a}}}\left\|v_{(\mathfrak{a}, \alpha)}\right\|^{2}\right] & =: \operatorname{SDP}(\mathfrak{I}) & \\ \text { subject to } \quad\left\langle v_{\left(S_{1}, \alpha_{1}\right)}, v_{\left(S_{2}, \alpha_{2}\right)}\right\rangle & =0 & \\ \left\langle v_{\left(S_{1}, \alpha_{1}\right)}, v_{\left(S_{2}, \alpha_{2}\right)}\right\rangle & =\left\langle v_{\left(S_{3}, \alpha_{3}\right)}, v_{\left(S_{4}, \alpha_{4}\right)}\right\rangle & \left.\forall \alpha_{1}\right|_{S_{1} \cap S_{2}} \neq\left.\alpha_{2}\right|_{S_{1} \cap S_{2}} \cup S_{2}=S_{3} \cup S_{4}, \alpha_{1} \circ \alpha_{2}=\alpha_{3} \circ \alpha_{4} \\ \sum_{j \in[q]}\left\|v_{(\{i\}, j)}\right\|^{2} & =1 & \\ \left\|v_{(\varnothing, \varnothing)}\right\| & =1 & \\ & & \end{array}$

For any set $S$ with $|S| \leq t$, the vectors $v_{(S, \alpha)}$ induce a probability distribution $\mu_{S}$ over $[q]^{S}$ such that the assignment $\alpha \in[q]^{S}$ appears with probability $\left\|v_{(S, \alpha)}\right\|^{2}$. Moreover, these distributions are consistent on intersections i.e., for $T \subseteq S \subseteq[n]$, we have $\mu_{S \mid T}=\mu_{T}$, where $\mu_{S \mid T}$ denotes the restriction of the distribution $\mu_{S}$ to the set $T$. We use these distributions to define a collection of random variables $\mathbf{Y}_{1}, \ldots, \mathbf{Y}_{n}$ taking values in $[q]$, such that for any set $S$ with $|S| \leq t$, the collection of variables $\left\{\mathbf{Y}_{i}\right\}_{i \in S}$ have a joint distribution $\mu_{S}$. Note that the entire collection $\left(\mathbf{Y}_{1}, \ldots, \mathbf{Y}_{n}\right)$ may not have a joint distribution: this property is only true for sub-collections of size $t$. We will refer to the collection $\left(\mathbf{Y}_{1}, \ldots, \mathbf{Y}_{n}\right)$ as a $t$-local ensemble of random variables.

We also have that that for any $T \subseteq[n]$ with $|T| \leq t-2$, and any $\beta \in[q]^{T}$, we can define a $(t-|T|)$-local ensemble $\left(\mathbf{Y}_{1}^{\prime}, \ldots, \mathbf{Y}_{n}^{\prime}\right)$ by "conditioning" the local distributions on the event $\mathbf{Y}_{T}=\beta$, where $Y_{T}$ is shorthand for the collection $\left\{\mathbf{Y}_{i}\right\}_{i \in T}$. For any $S$ with $|S| \leq$ $t-|T|$, we define the distribution of $\mathbf{Y}_{S}^{\prime}$ as $\mu_{S}^{\prime}:=\mu_{S \cup T} \mid\left\{\mathbf{Y}_{T}=\beta\right\}$. Finally, the semidefinite program also ensures that for any such conditioning, the conditional covariance matrix

$$
\mathrm{M}_{\left(S_{1}, \alpha_{1}\right)\left(S_{2}, \alpha_{2}\right)}=\operatorname{Cov}\left(\mathbf{1}\left[\mathbf{Y}_{S_{1}}^{\prime}=\alpha_{1}\right], \mathbf{1}\left[\mathbf{Y}_{S_{2}}^{\prime}=\alpha_{2}\right]\right)
$$

is positive semidefinite, where $\left|S_{1}\right|,\left|S_{2}\right| \leq(t-|T|) / 2$. Here, for each pair $S_{1}, S_{2}$ the covariance is computed using the joint distribution $\mu_{S_{1} \cup S_{2}}^{\prime}$. The PSD-ness be easily verified by noticing that the above matrix can be written as the Gram matrix of the vectors

$$
w_{(S, \alpha)}:=\frac{1}{\left\|v_{(T, \beta)}\right\|} \cdot v_{(T \cup S, \beta \circ \alpha)}-\frac{\left\|v_{(T \cup S, \beta \circ \alpha)}\right\|^{2}}{\left\|v_{(T, \beta)}\right\|^{3}} \cdot v_{(T, \beta)}
$$

In this paper, we will only consider $t$-local ensembles such that for every conditioning on a set of size at most $t-2$, the conditional covariance matrix is PSD. We will refer to these as $t$-local PSD ensembles. We will also need a simple corollary of the above definitions. 
Fact 2.7. Let $\left(\mathbf{Y}_{1}, \ldots, \mathbf{Y}_{n}\right)$ be a t-local PSD ensemble, and let $X$ be any simplicial complex with $X(1)=[n]$. Then, for all $s \leq t / 2$, the collection $\left\{\mathbf{Y}_{\mathfrak{a}}\right\}_{\mathfrak{a} \in X(\leq s)}$ is a $(t / s)$-local PSD ensemble, where $X(\leq s)=\bigcup_{i=1}^{s} X(i)$.

For random variables $\mathbf{Y}_{S}$ in a $t$-local PSD ensemble, we use the notation $\left\{\mathbf{Y}_{S}\right\}$ to denote the distribution of $\mathbf{Y}_{S}$ (which exists when $|S| \leq t$ ). We also define $\operatorname{Var}\left[\mathbf{Y}_{S}\right]$ as $\sum_{\alpha \in[q]^{s}} \operatorname{Var}\left[\mathbf{1}\left[\mathbf{Y}_{S}=\alpha\right]\right]$.

\section{Proof Overview: Approximating MAX 4-XOR}

We consider a simple example of a specific $k$-CSP, which captures most of the key ideas in our proof. Let $\mathfrak{I}$ be an unweighted instance of 4-XOR on $n$ Boolean variables. Let $H$ be a 4uniform hypergraph on vertex set $[n]$, with a hyperedge corresponding to each constraint i.e., each $\mathfrak{a}=\left\{i_{1}, i_{2}, i_{3}, i_{4}\right\} \in H$ corresponds to a constraint in $\mathfrak{I}$ of the form

$$
x_{i_{1}}+x_{i_{2}}+x_{i_{3}}+x_{i_{4}}=b_{\mathfrak{a}}(\bmod 2),
$$

for some $b_{\mathfrak{a}} \in\{0,1\}$. Let $X$ denote the constraint complex for the instance $\mathfrak{I}$ such that $X(1)=[n], X(4)=H$ and let $\Pi_{1}, \ldots, \Pi_{4}$ be the associated distributions (with $\Pi_{4}$ being uniform on $H$ ).

Local vs global correlation: the BRS strategy. We first recall the strategy used by [BRS11], which also suggests a natural first step for our proof. Given a 2-CSP instance with an associated graph $G$, and a $t$-local PSD ensemble $\mathbf{Y}_{1}, \ldots, \mathbf{Y}_{n}$ obtained from the SoS relaxation, they consider if the "local correlation" of the ensemble is small across the edges of $G$ (which correspond to constraints) i.e.,

$$
\underset{\{i, j\} \sim G}{\mathbb{E}}\left[\left\|\left\{\mathbf{Y}_{i} \mathbf{Y}_{j}\right\}-\left\{\mathbf{Y}_{i}\right\}\left\{\mathbf{Y}_{j}\right\}\right\|_{1}\right] \leq \varepsilon .
$$

If the local correlation is indeed small, we easily produce an assignment achieving a value SDP $-\varepsilon$ in expectation, simply by rounding each variable $x_{i}$ independently according to the distribution $\left\{\mathbf{Y}_{i}\right\}$. On the other hand, if this is not satisfied, they show (as a special case of their proof) that if $G$ is an expander with second eigenvalue $\lambda \leq c \cdot\left(\varepsilon^{2} / q^{2}\right)$, then variables also have a high "global correlation", between a typical pair $(i, j) \in[n]^{2}$. Here, $q$ is the alphabet size and $c$ is a fixed constant. They use this to show that for $\left(\mathbf{Y}_{1}^{\prime}, \ldots, \mathbf{Y}_{n}^{\prime}\right)$ obtained by conditioning on the value of a randomly chosen $\mathbf{Y}_{i_{0}}$, we have

$$
\underset{i}{\mathbb{E}}\left[\operatorname{Var}\left[\mathbf{Y}_{i}\right]\right]-\underset{i_{0}, \mathbf{Y}_{i_{0}}}{\mathbb{E}} \mathbb{i}\left[\operatorname{Var}\left[\mathbf{Y}_{i}^{\prime}\right]\right] \geq \Omega\left(\varepsilon^{2} / q^{2}\right)
$$

where the expectations over $i$ and $i_{0}$ are both according to the stationary distribution on the vertices of $G$. Since the variance is bounded between 0 and 1 , this essentially shows that the local correlation must be at most $\varepsilon$ after conditioning on a set of size $O\left(q^{2} / \varepsilon^{2}\right)$ (although the actual argument requires a bit more care and needs to condition on a somewhat larger set).

Extension to 4-XOR. As in [BRS11], we check if the $t$-local PSD ensemble $\left(\mathbf{Y}_{1}, \ldots, \mathbf{Y}_{n}\right)$ obtained from the SDP solution satisfies

$$
\underset{\left\{i_{1}, i_{2}, i_{3}, i_{4}\right\} \in H}{\mathbb{E}}\left[\left\|\left\{\mathbf{Y}_{i_{1}} \mathbf{Y}_{i_{2}} \mathbf{Y}_{i_{3}} \mathbf{Y}_{i_{4}}\right\}-\left\{\mathbf{Y}_{i_{1}}\right\}\left\{\mathbf{Y}_{i_{2}}\right\}\left\{\mathbf{Y}_{i_{3}}\right\}\left\{\mathbf{Y}_{i_{4}}\right\}\right\|_{1}\right] \leq \varepsilon .
$$


As before, independently sampling each $x_{i}$ from $\left\{\mathbf{Y}_{i}\right\}$ gives an expected value at least $\mathrm{SDP}-\varepsilon$ in this case. If the above inequality is not satisfied, an application of triangle inequality gives

$$
\underset{\left\{i_{1}, i_{2}, i_{3}, i_{4}\right\} \in H}{\mathbb{E}}\left[\begin{array}{l}
\left\|\left\{\mathbf{Y}_{i_{1}} \mathbf{Y}_{i_{2}} \mathbf{Y}_{i_{3}} \mathbf{Y}_{i_{4}}\right\}-\left\{\mathbf{Y}_{i_{1}} \mathbf{Y}_{i_{2}}\right\}\left\{\mathbf{Y}_{i_{3}} \mathbf{Y}_{i_{4}}\right\}\right\|_{1}+ \\
\left\|\left\{\mathbf{Y}_{i_{1}} \mathbf{Y}_{i_{2}}\right\}-\left\{\mathbf{Y}_{i_{1}}\right\}\left\{\mathbf{Y}_{i_{2}}\right\}\right\|_{1}+\left\|\left\{\mathbf{Y}_{i_{3}} \mathbf{Y}_{i_{4}}\right\}-\left\{\mathbf{Y}_{i_{3}}\right\}\left\{\mathbf{Y}_{i_{4}}\right\}\right\|_{1}
\end{array}\right]>\varepsilon .
$$

Symmetrizing over all orderings of $\left\{i_{1}, i_{2}, i_{3}, i_{4}\right\}$, we can write the above as

$$
\varepsilon_{2}+2 \cdot \varepsilon_{1}>\varepsilon,
$$

which gives $\max \left\{\varepsilon_{1}, \varepsilon_{2}\right\} \geq \varepsilon / 3$. Here,

$$
\begin{aligned}
\varepsilon_{1} & :=\underset{\left\{i_{1}, i_{2}\right\} \sim \Pi_{2}}{\mathbb{E}}\left[\left\|\left\{\mathbf{Y}_{i_{1}} \mathbf{Y}_{i_{2}}\right\}-\left\{\mathbf{Y}_{i_{1}}\right\}\left\{\mathbf{Y}_{i_{2}}\right\}\right\|_{1}\right], \text { and } \\
\varepsilon_{2}: & : \underset{\left\{i_{1}, i_{2}, i_{3}, i_{4}\right\} \sim \Pi_{4}}{\mathbb{E}}\left[\left\|\left\{\mathbf{Y}_{i_{1}} \mathbf{Y}_{i_{2}} \mathbf{Y}_{i_{3}} \mathbf{Y}_{i_{4}}\right\}-\left\{\mathbf{Y}_{i_{1}} \mathbf{Y}_{i_{2}}\right\}\left\{\mathbf{Y}_{i_{3}} \mathbf{Y}_{i_{4}}\right\}\right\|_{1}\right] \\
& =\underset{\left\{i_{1}, i_{2}, i_{3}, i_{4}\right\} \sim \Pi_{4}}{\mathbb{E}}\left[\left\|\left\{\mathbf{Y}_{\left\{i_{1}, i_{2}\right\}} \mathbf{Y}_{\left\{i_{3}, i_{4}\right\}}\right\}-\left\{\mathbf{Y}_{\left\{i_{1}, i_{2}\right\}}\right\}\left\{\mathbf{Y}_{\left\{i_{3}, i_{4}\right\}}\right\}\right\|_{1}\right] .
\end{aligned}
$$

As before, $\varepsilon_{1}$ measures the local correlation across edges of a weighted graph $G_{1}$ with vertex set $X(1)=[n]$ and edge-weights given by $\Pi_{2}$. Also, $\varepsilon_{2}$ measures the analogous quantity for a graph $G_{2}$ with vertex set $X(2)$ (pairs of variables occurring in constraints) and edge-weights given by $\Pi_{4}$.

Recall that the result from [BRS11] can be applied to any graph $G$ over variables in a 2-local PSD ensemble, as long as the $\sigma_{2}(G)$ is small. Since $\left\{\mathbf{Y}_{i}\right\}_{i \in[n]}$ and $\left\{\mathbf{Y}_{\mathfrak{s}}\right\}_{\mathfrak{s} \in X(2)}$ are both $(t / 2)$-local PSD ensembles (by Fact 2.7), we will apply the result to the graph $G_{1}$ on the first ensemble and $G_{2}$ on the second ensemble. We consider the potential

$$
\Phi\left(\mathbf{Y}_{1}, \ldots, \mathbf{Y}_{n}\right):=\underset{i \sim \Pi_{1}}{\mathbb{E}}\left[\operatorname{Var}\left[\mathbf{Y}_{i}\right]\right]+\underset{\mathfrak{s} \sim \Pi_{2}}{\mathbb{E}}\left[\operatorname{Var}\left[\mathbf{Y}_{\mathfrak{s}}\right]\right]
$$

Since local correlation is large along at least one of the graphs $G_{1}$ and $G_{2}$, using the above arguments (and the non-decreasing nature of variance under conditioning) it is easy to show that in expectation over the choice of $\left\{i_{0}, j_{0}\right\} \sim \Pi_{2}$ and $\beta \in[q]^{2}$ chosen from $\left\{\mathbf{Y}_{\left\{i_{0}, j_{0}\right\}}\right\}$, the conditional ensemble $\left(\mathbf{Y}_{1}^{\prime}, \ldots, \mathbf{Y}_{n}^{\prime}\right)$ satisfies

$$
\Phi\left(\mathbf{Y}_{1}, \ldots, \mathbf{Y}_{n}\right)-\underset{i_{0}, j_{0}, \beta}{\mathbb{E}}\left[\Phi\left(\mathbf{Y}_{1}^{\prime}, \ldots, \mathbf{Y}_{n}^{\prime}\right)\right]=\Omega\left(\varepsilon^{2}\right)
$$

provided $G_{1}$ and $G_{2}$ satisfy $\sigma_{2}\left(G_{1}\right), \sigma_{2}\left(G_{2}\right) \leq c \cdot \varepsilon^{2}$ for an appropriate constant $c$.

The bound on the eigenvalue of $G_{1}$ follows simply from the fact that it is the skeleton of $X$, which is a $\gamma$-HDX. Obtaining bounds on the eigenvalues of $G_{2}$ and similar higherorder graphs, constitutes much of the technical part of this paper. Note that for a random sparse instance of MAX 4-XOR, the graph $G_{2}$ will be a matching with high probability (since $\left\{i_{1}, i_{2}\right\}$ in a constraint will only be connected to $\left\{i_{3}, i_{4}\right\}$ in the same constraint). However, we show that in case of a $\gamma$-HDX, this graph has second eigenvalue $O(\gamma)$. We analyze these graphs in terms of modified high-dimensional random walks, which we call "swap walks".

We remark that our potential and choice of a "seed set" of variables to condition on, is slightly different from [BRS11]. To decrease the potential function above, we need that for 
each level $X(i)$ ( $i=1,2$ in the example above) the seed set must contain sufficiently many independent samples from $X(i)$ sampled according to $\Pi_{i}$. This can be ensured by drawing independent samples from the top level $X(k)$ (though $X(2)$ suffices in the above example). In contrast, the seed set in [BRS11] consists of random samples from $\Pi_{1}$.

Analyzing Swap Walks. The graph $G_{2}$ defined above can be thought of as a random walk on $X(2)$, which starts at a face $\mathfrak{s} \in X(2)$, moves up to a face (constraint) $\mathfrak{s}^{\prime} \in X(4)$ containing it, and then descends to a face $\mathfrak{t} \in X(2)$ such that $\mathfrak{t} \subset \mathfrak{s}^{\prime}$ and $\mathfrak{s} \cap \mathfrak{t}=\varnothing$ i.e., the walk "swaps out" the elements in $\mathfrak{s}$ for other elements in $\mathfrak{s}^{\prime}$. Several walks considered on simplicial complexes allow for the possibility of a non-trivial intersection, and hence have second eigenvalue lower bounded by a constant. On the other hand, swap walks completely avoid any laziness and thus turn out to have eigenvalues which can be made arbitrarily small. To understand the eigenvalues for this walk, we will express it in terms of other canonical walks defined on simplicial complexes.

Recall that the up and down operators can be used to define random walks on simplicial complexes. The up operator $\mathrm{U}_{i}: C^{i} \rightarrow C^{i+1}$ defines a walk that moves down from a face $\mathfrak{s} \in X(i+1)$ to a random face $\mathfrak{t} \in X(i), \mathfrak{t} \subset \mathfrak{s}$ (the operator thus "lifts" a function in $C^{i}$ to a function in $\left.C^{i+1}\right)$. Similarly, the down operator $D_{i}: C^{i} \rightarrow C^{i-1}$ moves up from a face $\mathfrak{s} \in X(i-1)$ to $\mathfrak{t} \in X(i), \mathfrak{t} \supset s$, with probability $\Pi_{i}(\mathfrak{t}) /\left(i \cdot \Pi_{i-1}(\mathfrak{s})\right)$. These can be used to define a canonical random walk

$$
\mathrm{N}_{2,2}^{(u)}:=\mathrm{D}_{3} \cdots \mathrm{D}_{u+2} \mathrm{U}_{u+1} \cdots \mathrm{U}_{2}, \quad \mathrm{~N}_{2,2}^{(u)}: C^{2} \rightarrow C^{2},
$$

which moves from up for $u$ steps $\mathfrak{s} \in X(2)$ to $\mathfrak{s}^{\prime} \in X(u+2)$, and then descends back to $\mathfrak{t} \in$ $X(2)$. Such walks were analyzed optimally by Dinur and Kaufman [DK17], who proved that $\lambda_{2}\left(\mathrm{~N}_{2,2}^{(u)}\right)=2 /(u+2) \pm O_{u}(\gamma)$ when $X$ is a $\gamma$-HDX. Thus, while this walk gives an expanding graph with vertex set $X(2)$, the second eigenvalue cannot be made arbitrarily small for a fixed $u$ (recall that we are interested in showing that $\sigma_{2}\left(G_{2}\right) \leq c \cdot \varepsilon^{2}$ ). However, note that we are only interested in $\mathrm{N}_{2,2}^{(2)}$ conditioned on the event that the two elements from $\mathfrak{s}$ are "swapped out" with new elements in the final set $\mathfrak{t}$ i.e., $\mathfrak{s} \cap \mathfrak{t}=\varnothing$. We define

$$
\mathrm{S}_{2,2}^{(u, j)}(\mathfrak{s}, \mathfrak{t}):= \begin{cases}\frac{\left(\begin{array}{c}
u+2 \\
2
\end{array}\right)}{\left(\begin{array}{c}
u \\
j
\end{array}\right) \cdot\left(\begin{array}{c}
2 \\
2-j
\end{array}\right)} \cdot \mathrm{N}_{2,2}^{(u)} & \text { if }|\mathfrak{t} \backslash \mathfrak{s}|=j \\
0 & \text { otherwise }\end{cases}
$$

where the normalization is to ensure stochasticity of the matrix. In this notation, the graph $G_{2}$ corresponds to the random-walk matrix $S_{2,2}^{(2,2)}$. We show that while $\sigma_{2}\left(\mathrm{~N}_{2,2}^{(2)}\right) \approx 1 / 2$, we have that $\sigma_{2}\left(\mathrm{~S}_{2,2}^{(2,2)}\right)=O(\gamma)$. We first write the canonical walks in terms of the swap walks. Note that

$$
\mathrm{N}_{2,2}^{(2)}=\frac{1}{6} \cdot \mathrm{I}+\frac{2}{3} \cdot \mathrm{S}_{2,2}^{(2,1)}+\frac{1}{6} \cdot \mathrm{S}_{2,2}^{(2,2)},
$$

since the "descent" step from $\mathfrak{s}^{\prime} \in X(4)$ containing $\mathfrak{s} \in X(2)$, produces a $\mathfrak{t} \in X(2)$ which "swaps out" 0,1 and 2 elements with probabilities 1/6,2/3 and 1/6 respectively. Similarly,

$$
\mathrm{N}_{2,2}^{(1)}=\frac{1}{3} \cdot \mathrm{I}+\frac{2}{3} \cdot \mathrm{S}_{2,2}^{(1,1)} \text {. }
$$

Finally, we use the fact (proved in Section 4) that while the canonical walks do depend on the "height" $u$ (i.e., $\mathrm{N}_{2,2}^{(u)} \neq \mathrm{N}_{2,2}^{\left(u^{\prime}\right)}$ ) the swap walks (for a fixed number of swaps $j$ ) are 
independent of the height to which they ascend! In particular, we have

$$
\mathrm{S}_{2,2}^{(2,1)}=\mathrm{S}_{2,2}^{(1,1)} \text {. }
$$

Using these, we can derive an expression for the swap walk $\mathrm{S}_{2,2}^{(2,2)}$ as

$$
\mathrm{S}_{2,2}^{(2,2)}=\mathrm{I}+6 \cdot \mathrm{N}_{2,2}^{(2)}-6 \cdot \mathrm{N}_{2,2}^{(1)}=\mathrm{I}+6 \cdot\left(\mathrm{D}_{3} \mathrm{D}_{4} \mathrm{U}_{3} \mathrm{U}_{2}-\mathrm{D}_{3} \mathrm{U}_{2}\right)
$$

To understand the spectrum of operators such as the ones given by the above expression, we use the beautiful machinery for harmonic analysis over HDXs (and more generally over expanding posets) developed by Dikstein et al. [DDFH18]. They show how to decompose the spaces $C^{k}$ into approximate eigenfunctions for operators of the form DU. Using these decompositions and the properties of expanding posets, we can show that distinct eigenvalues of the above operator are approximately the same (up to $O(\gamma)$ errors) when analyzing the walks on the complete complex. Finally, we use the fact that swap walks in a complete complex correspond to Kneser graphs (for which the eigenvectors and eigenvalues are well-known) to show that $\lambda_{2}\left(\mathrm{~S}_{2,2}^{(2,2)}\right)=O(\gamma)$.

Splittable CSPs and high-dimensional threshold rank. We note that the ideas used above can be generalized (at least) in two ways. In the analysis of distance from product distribution for a 4-tuple of random variables forming a contraint, we split it in 2-tuples. In general, we can choose to split tuples in a $k$-CSP instance along any binary tree $\mathcal{T}$ with $k$ leaves, with each parent node corresponding to a swap walk between tuples forming its children. Finally, the analysis from [BRS11] also works if the each of the swap walks in some $\mathcal{T}$ have a bounded number (say $r$ ) of eigenvalues above some threshold $\tau$, which provide a notion of high-dimensional threshold rank for hypergraphs. We refer to such an instance as a $(\mathcal{T}, \tau, r)$-splittable.

The arguments sketched above show that high-dimensional expanders are $(\mathcal{T}, O(\gamma), 1)$ splittable for all $\mathcal{T}$. Since the knowledge of $\mathcal{T}$ is only required in our analysis and not in the algorithm, we say that $\operatorname{rank}_{\tau}(\mathfrak{I}) \leq r$ (or that $\mathfrak{I}$ is $(\tau, r)$-splittable) if $\mathfrak{I}$ is $(\mathcal{T}, \tau, r)$ splittable for any $\mathcal{T}$. We defer the precise statement of results for $(\tau, r)$-splittable instances to Section 7.

\section{Walks}

It is important to note that both $\mathrm{U}_{i}$ and $\mathrm{D}_{i+1}$ can be thought of as row-stochastic matrices, i.e. we can think of them as the probability matrices describing the movement of a walk from $X(i+1)$ to $X(i)$; and from $X(i)$ to $X(i+1)$ respectively. More concretely, we will think

$$
\left[\mathrm{D}_{i+1}^{\top} e_{\mathfrak{s}}\right](\mathfrak{t})=\mathbb{P}[\text { the walk moves up from } \mathfrak{s} \in X(i) \text { to } \mathfrak{t} \in X(i+1)]
$$

and similarly

$$
\left[U_{i}^{\top} e_{\mathfrak{t}}\right](\mathfrak{s})=\mathbb{P}[\text { the walk moves down from } \mathfrak{t} \in X(i+1) \text { to } \mathfrak{s} \in X(i)] .
$$

By referring to the definition of the up and down operators in Section 2, it is easy to verify that

$$
\left[\mathrm{D}_{i+1}^{\top} e_{\mathfrak{s}}\right](\mathfrak{t})=\mathbf{1}[\mathfrak{t} \supseteq \mathfrak{s}] \cdot \frac{1}{i+1} \frac{\Pi_{i+1}(\mathfrak{t})}{\prod_{i}(\mathfrak{s})} \quad \text { and } \quad\left[\mathrm{U}_{i}^{\top} e_{\mathfrak{t}}\right](\mathfrak{s})=\mathbf{1}[\mathfrak{s} \subseteq \mathfrak{t}] \cdot \frac{1}{i+1}
$$


It is easy to see that our notion of random walk respects the probability distributions $\Pi_{j}$, i.e. we have

$$
\mathrm{U}_{i}^{\top} \Pi_{i+1}=\Pi_{i} \quad \text { and } \quad \mathrm{D}_{i+1}^{\top} \Pi_{i}=\Pi_{i+1}
$$

i.e., randomly moving up from a sample of $\Pi_{j}$ gives a sample of $\Pi_{j+1}$ and similarly, moving down from a sample of $\Pi_{j+1}$ results in a sample of $\Pi_{j}$.

Instead of going up and down by one dimension, one can try going up or down by multiple dimensions since $\left(\mathrm{D}_{i+1} \cdots \mathrm{D}_{i+\ell}\right)$ and $\left(\mathrm{U}_{i+\ell} \cdots \mathrm{U}_{i}\right)$ are still row-stochastic matrices. Further, the corresponding probability vectors still have intuitive explanations in terms of the distributions $\Pi_{j}$. For a face $\mathfrak{s} \in X(k)$, we introduce the notation

$$
p_{\mathfrak{s}}^{(u)}=\left(\mathrm{D}_{k+1} \cdots \mathrm{D}_{k+u}\right)^{\top} e_{\mathfrak{s}}
$$

where we take $p_{\mathfrak{s}}^{(0)}=e_{\mathfrak{s}}$. This notation will be used to denote the probability distribution of the up-walk starting from $\mathfrak{s} \in X(k)$ and ending in a random face $\mathfrak{t} \in X(k+u)$ satisfying $\mathfrak{t} \supseteq \mathfrak{s}$.

Note that the following Lemma together with Proposition 2.4 implies that $p_{\mathfrak{s}}^{(u)}$ is indeed a probability distribution.

Proposition 4.1. For $\mathfrak{s} \in X(k)$ and $\mathfrak{a} \in X(k+u)$ one has,

$$
p_{\mathfrak{s}}^{(u)}(\mathfrak{a})=\mathbf{1}[\mathfrak{a} \supseteq \mathfrak{s}] \cdot \frac{1}{\left(\begin{array}{c}
k+u \\
u
\end{array}\right)} \cdot \frac{\Pi_{k+u}(\mathfrak{a})}{\Pi_{k}(\mathfrak{s})} .
$$

Proof. Notice that for $u=0$, the statement holds trivially. We assume that there exists some $u \geq 0$ that satisfies

$$
p_{\mathfrak{s}}^{(u)}(\mathfrak{a})=\mathbf{1}[\mathfrak{a} \supseteq \mathfrak{s}] \cdot \frac{1}{\left(\begin{array}{c}
k+u \\
u
\end{array}\right)} \cdot \frac{\Pi_{k+u}(\mathfrak{a})}{\prod_{k}(\mathfrak{s})}
$$

for all $\mathfrak{a} \in X(k+u)$.

For $\mathfrak{b} \in X(k+(u+1))$ one has,

$$
p_{\mathfrak{s}}^{(u+1)}(\mathfrak{b})=\left[\mathrm{D}_{k+u+1}^{\top} p_{\mathfrak{s}}^{(u)}\right](\mathfrak{b})=\frac{1}{k+u+2} \cdot \sum_{x \in \mathfrak{b}} \frac{\Pi_{k+u+1}(\mathfrak{b})}{\Pi_{k+u}(\mathfrak{b} \backslash\{x\})} \cdot p_{\mathfrak{s}}^{(u)}(\mathfrak{b} \backslash\{x\}) .
$$

Plugging in the induction assumption, this implies

$$
\begin{aligned}
p_{\mathfrak{s}}^{(u+1)}(\mathfrak{b}) & \left.=\frac{1}{(k+u+1)} \cdot \sum_{x \in \mathfrak{b}} \frac{\Pi_{k+u+1}(\mathfrak{b})}{\Pi_{k+u}(\mathfrak{b} \backslash\{x\})} \cdot(\mathbf{1}[(\mathfrak{b} \backslash\{x)\}) \supseteq \mathfrak{s}] \cdot \frac{1}{\left(\begin{array}{c}
k+u \\
u
\end{array}\right)} \cdot \frac{\Pi_{k+u}(\mathfrak{b} \backslash\{x\})}{\Pi_{k}(\mathfrak{s})}\right), \\
& =\frac{1}{(k+u+1)} \cdot \frac{1}{\left(\begin{array}{c}
k+u \\
u
\end{array}\right)} \cdot \sum_{x \in \mathfrak{b}} \mathbf{1}[\mathfrak{b} \backslash\{x\} \supseteq \mathfrak{s}] \cdot \frac{\Pi_{k+u+1}(\mathfrak{b})}{\Pi_{k}(\mathfrak{s})} .
\end{aligned}
$$

First, note that the up-walk only hits the faces that contain $\mathfrak{s}$, otherwise $\mathbf{1}[\mathfrak{b} \backslash\{x\} \supseteq \mathfrak{s}]=0$.

Suppose therefore $\mathfrak{b} \in X(k+u+1)$ satisfies $\mathfrak{b} \supseteq \mathfrak{s}$. Since there are precisely $(u+1)$ indices whose deletion still preserves the containment of $\mathfrak{s}$, we can write

$$
\begin{aligned}
p_{\mathfrak{s}}^{(u+1)}(\mathfrak{b}) & =\mathbf{1}[\mathfrak{b} \supseteq \mathfrak{s}] \cdot \frac{u+1}{k+u+1} \cdot \frac{1}{\left(\begin{array}{c}
k+u \\
u
\end{array}\right)} \frac{\Pi_{k+u+1}(\mathfrak{b})}{\prod_{k}(\mathfrak{s})}, \\
& =\mathbf{1}[\mathfrak{b} \supseteq \mathfrak{s}] \cdot \frac{1}{\left(\begin{array}{c}
k+u+1 \\
u+1
\end{array}\right)} \cdot \frac{\Pi_{k+u+1}(\mathfrak{b})}{\prod_{k}(\mathfrak{s})} .
\end{aligned}
$$

Thus, proving the proposition. 
Similarly, we introduce the notation $q_{\mathfrak{a}}^{(u)}$, as

$$
q_{\mathfrak{a}}^{(u)}(\mathfrak{s})=\left(\mathrm{U}_{k+u-1} \cdots \mathrm{U}_{k}\right)^{\top} e_{\mathfrak{s}}
$$

i.e. for the probability distribution of the down-walk starting from $\mathfrak{a} \in X(k+u)$ and ending in a random face of $X(k)$ contained in $\mathfrak{a}$. The following can be verified using Proposition 4.1 , and the fact that $\left(\mathrm{U}_{k+u-1} \cdots \mathrm{U}_{k}\right)^{\dagger}=\mathrm{D}_{k+u} \cdots \mathrm{D}_{k+1}$.

Corollary 4.2. Let $X(\leq d)$ be a simplicial complex, and $k, u \geq 0$ be parameters satisfying $k+u \leq$ $d$. For $\mathfrak{a} \in X(k+u)$ and $\mathfrak{s} \in X(k)$, one has

$$
q_{\mathfrak{a}}^{(u)}(\mathfrak{s})=\frac{1}{\left(\begin{array}{c}
k+u \\
u
\end{array}\right)} \cdot \mathbf{1}[\mathfrak{s} \subseteq \mathfrak{a}] .
$$

In the remainder of this section, we will try to construct more intricate walks on $X$ from $X(k)$ to $X(l)$.

\subsection{The Canonical and the Swap Walks on a Simplicial Complex}

Definition 4.3 (Canonical and Swap $u$-Walks). Let $d \geq 0, X(\leq d)$ be a simplicial complex, and $k, l, u \geq 0$ be parameters satisfying $l \leq k, u \leq l$ and $d \geq k+u$; where the constraints on these parameters are to ensure well-definedness. We will define the following random walks,

- canonical u-walk from $X(k)$ to $X(l)$. Let $\mathrm{N}_{k, l}^{(u)}$ be the (row-stochastic) Markov operator that represents the following random walk: Starting from a face $\mathfrak{s} \in X(k)$,

- (random ascent/up-walk) randomly move up a face $\mathfrak{s}^{\prime \prime} \in X(k+u)$ that contains $\mathfrak{s}$, where $\mathfrak{s}^{\prime \prime}$ is picked with probability

$$
p_{\mathfrak{s}}^{(u)}\left(\mathfrak{s}^{\prime \prime}\right)=\left[\left(\mathrm{D}_{k+1} \cdots \mathrm{D}_{k+u}\right)^{\top} e_{\mathfrak{s}}\right]\left(\mathfrak{s}^{\prime \prime}\right) .
$$

- (random descent/down-walk) go to a face $\mathfrak{s}^{\prime} \in X(l)$ picked uniformly among all the l-dimensional faces that are contained in $\mathfrak{s}^{\prime \prime}$, i.e., the set $\mathfrak{s}^{\prime}$ is picked with probability

$$
q_{\mathfrak{s}^{\prime \prime}}\left(\mathfrak{s}^{\prime}\right)=\mathbf{1}\left[\mathfrak{s}^{\prime} \subseteq \mathfrak{s}^{\prime \prime}\right] \cdot \frac{1}{\left(\begin{array}{c}
k+u \\
l
\end{array}\right)}=\left[\left(\mathrm{U}_{k+u-1} \cdots \mathrm{U}_{l}\right)^{\top} e_{\mathfrak{s}^{\prime \prime}}\right]\left(\mathfrak{s}^{\prime}\right)
$$

The operator $\mathrm{N}_{k, l}^{(u)}: C^{l} \rightarrow C^{k}$ satisfies the following equation,

$$
\mathrm{N}_{k, l}^{(u)}=\mathrm{D}_{k+1} \cdots \mathrm{D}_{k+u} \mathrm{U}_{k+u-1} \cdot \mathrm{U}_{k} \cdots \mathrm{U}_{l} .
$$

Notice that we have $\mathrm{N}_{k, k}^{(0)}=\mathrm{I}$, and $\mathrm{N}_{k, l}^{(0)}=\left(\mathrm{U}_{k-1} \ldots \mathrm{U}_{l}\right)$ for $l<k$.

- swapping walk from $X(k)$ to $X(l)$. Let $S_{k, l}$ be the Markov operator that represents the following random walk: Starting from a face $\mathfrak{s} \in X(k)$,

- (random ascent/up-walk) randomly move up to a face $\mathfrak{s}^{\prime \prime} \in X(k+l)$ that contains $\mathfrak{s}$, where as before $\mathfrak{s}^{\prime \prime}$ is picked with probability

$$
p_{\mathfrak{s}}^{(l)}\left(\mathfrak{s}^{\prime \prime}\right)=\left[\left(\mathrm{D}_{k+1} \cdots \mathrm{D}_{k+l+1}\right)^{\top} e_{\mathfrak{s}}\right]\left(\mathfrak{s}^{\prime \prime}\right) .
$$


- (deterministic descent) deterministically go to $\mathfrak{s}^{\prime}=\mathfrak{s}^{\prime \prime} \backslash \mathfrak{s} \in X(l)$.

For our applications, we will need to show that the walk $S_{k, l}$ has good spectral expansion whenever $X$ is a $d$-dimensional $\gamma$-expander, for $\gamma$ sufficiently small. To show this, we will relate the swapping walk operator $S_{k, l}$ on $X$ to the canonical random walk operators $\mathrm{N}_{k, l}^{(u)}$ (q.v. Lemma 4.4).

By the machinery of expanding posets (q.v. Section 5) it is possible to argue that the spectral expansion of the random walk operator $\mathrm{N}_{k, l}^{(u)}$ on a high dimensional expander will be close to that of the complete complex. This will allow us to conclude using the relation between the swapping walks and the canonical walks (q.v. Lemma 4.4) that the spectral expansion of the swapping walk on $X$, will be comparable with the spectral expansion of the swap walk on the complete complex. More precisely, we will show

Lemma 4.4 (Lemma 5.34). For any $d, k, l \geq 0$, and the complete simplicial simplicial complex $X(\leq d)$, one has the following: If $k \geq l \geq 0$ and $d \geq k+l$, we have

$$
\sigma_{2}\left(\mathrm{~S}_{k, l}\right)=O_{k, l}\left(\frac{1}{n}\right)
$$

Using these two, and the expanding poset machinery, we will conclude

Theorem 4.5 (Theorem 5.2 simplified). Let $X$ be a d-dimensional $\gamma$ expander. If $k \geq l \geq 0$ satisfy $d \geq l+k$ we have,

$$
\sigma_{2}\left(\mathrm{~S}_{k, l}\right)=O_{k, l}(\gamma)
$$

where $S_{k, l}$ is the swapping walk on $X$ from $X(k)$ to $X(l)$.

To prove Theorem 4.5 we will need to define an intermediate random walk that we will call the $j$-swapping $u$-walk from $X(k)$ to $X(l)$ :

Definition 4.6 ( $j$-swapping $u$-walk from $X(k)$ to $X(l)$ ). Given $d, u, j, k, l \geq 0$ satisfying $l \leq k$, $j \leq u, u \leq l$, and $d \geq k+u$. Let $\mathrm{S}_{k, l}^{(u, j)}$ be the Markov operator that represents the following random walk from $X(k)$ to $X(l)$ on a d-dimensional simplicial complex $X$ : Starting from $\mathfrak{s} \in X(k)$

- (random ascent/up-walk) randomly move up to a face $\mathfrak{s}^{\prime \prime} \in X(k+u)$ that contains $\mathfrak{s}$, where $\mathfrak{s}^{\prime \prime}$ is picked with probability

$$
p_{\mathfrak{s}}^{(u)}\left(\mathfrak{s}^{\prime \prime}\right)=\left[\left(\mathrm{D}_{k+1} \cdots \mathrm{D}_{k+u}\right)^{\top} e_{\mathfrak{s}}\right]\left(\mathfrak{s}^{\prime \prime}\right) .
$$

- (conditioned descent) go to a face $\mathfrak{s}^{\prime} \in X(l)$ sampled uniformly among all the subsets of $\mathfrak{s}^{\prime \prime} \in X(k+u)$ that have intersection $j$ with $\mathfrak{s}^{\prime \prime} \backslash \mathfrak{s}$, i.e. $\left|\mathfrak{s}^{\prime} \cap\left(\mathfrak{s}^{\prime \prime} \backslash \mathfrak{s}\right)\right|=j$.

Notice that $\mathrm{S}_{k, l}=\mathrm{S}_{k, l}^{(l, l)}$ for any $k$ and $\mathrm{I}=\mathrm{S}_{k, k}^{(u, 0)}$ for any $u$.

Remark 4.7. We will prove that the parameter $u$ does not effect the swapping walk $\mathrm{S}_{k, l}^{(u, j)}$ so long as $u \geq j$, i.e. for all $u, u^{\prime} \geq j$ we have $S_{k, l}^{\left(u^{\prime}, j\right)}=S_{k, l}^{(u, j)}$. Thus, we will often write $S_{k, l}^{(j)}$ for $S_{k, l}^{(j, j)}$. 


\subsection{Swap Walks are Height Independent}

Recall that the swap walk $\mathrm{S}_{k, l}^{(u, j)}$ is the conditional walk defined in terms of $\mathrm{N}_{k, l}^{(u)}$ where $\mathfrak{s} \in X(k)$ is connected to $\mathfrak{t} \in X(l)$ only if $|\mathfrak{t} \backslash \mathfrak{s}|=j$. The parameter $u$ is called the height of the walk, namely the number of times it moves up. Since up and down operators have second singular value bounded away from 1 , the second singular value of $\mathrm{N}_{k, l}^{(u)}$ shrinks as $u$ increases. In other words, the operator $\mathrm{N}_{k, l}^{(u)}$ depends on the height $u$. Surprisingly, the walk $\mathrm{S}_{k, l}^{(u, j)}$ which is defined in terms of $\mathrm{N}_{k, l}^{(u)}$ does not depend on the particular choice of $u$ as long as it is well defined. More precisely, we have the following result.

Lemma 4.8. If $X$ is a d-dimensional simplicial complex, $0 \leq l \leq k$, and $u, u^{\prime} \in[j, d-k]$, then

$$
\mathrm{S}_{k, l}^{(u, j)}=\mathrm{S}_{k, l}^{\left(u^{\prime}, j\right)}
$$

In order to obtain Lemma 4.8, we will need a simple proposition:

Proposition 4.9. Let $\mathfrak{s} \in X(k), \mathfrak{s}^{\prime} \subseteq \mathfrak{s}$ and $\left|t^{\prime}\right|=j$. Suppose $\mathfrak{s}^{\prime} \sqcup \mathfrak{t}^{\prime} \in X(l)$. Then, we have

$$
\mathrm{S}_{k, l}^{(u, j)}\left(\mathfrak{s}, \mathfrak{s}^{\prime} \sqcup \mathfrak{t}^{\prime}\right)=\frac{1}{\left(\begin{array}{c}
k \\
l-j
\end{array}\right) \cdot\left(\begin{array}{c}
u \\
j
\end{array}\right)} \cdot \sum_{\substack{\left.\mathfrak{a} \in X(k+u): \\
\mathfrak{a} \supseteq(\mathfrak{s} \sqcup\lrcorner^{\prime}\right)}} p_{\mathfrak{s}}^{(u)}(\mathfrak{a}) .
$$

Proof. The only way of picking $\mathfrak{s}^{\prime} \sqcup \mathfrak{t}^{\prime}$ at the descent step is picking some $\mathfrak{a} \in X(k+u)$ that contains $\mathfrak{s}^{\prime} \sqcup \mathfrak{t}^{\prime}$ in the ascent step. The probability of this happening is precisely,

$$
p_{1}=\sum_{\substack{\mathfrak{a} \in X(k+u): \\ \mathfrak{a} \supseteq\left(\mathfrak{s} \sqcup \mathfrak{t}^{\prime}\right)}} p_{\mathfrak{s}}^{(u)}(\mathfrak{a}) .
$$

Suppose we are at a set $\mathfrak{a}=\mathfrak{s} \sqcup \mathfrak{t}$, such that $\mathfrak{t} \supseteq \mathfrak{t}^{\prime}$ and $\mathfrak{s} \cap \mathfrak{t}=\varnothing$. Now, the probability of the descent step ending at $\mathfrak{s}^{\prime} \sqcup \mathfrak{t}^{\prime}$ is the probability of a randomly sampled $(l-j)$-elemented subset of $\mathfrak{s}$ being $\mathfrak{s}^{\prime}$ and the probability of a randomly sampled $j$-elemented subset of $\mathfrak{t}$ being $\mathfrak{t}^{\prime}$. It can be verified that this probability is

$$
p_{2}=\frac{1}{\left(\begin{array}{c}
k \\
l-j
\end{array}\right) \cdot\left(\begin{array}{c}
u \\
j
\end{array}\right)} .
$$

By law of total probability we establish that

$$
\mathrm{S}_{k, l}^{(u, j)}\left(\mathfrak{s}, \mathfrak{s}^{\prime} \sqcup \mathfrak{t}^{\prime}\right)=p_{1} \cdot p_{2}=\frac{1}{\left(\begin{array}{c}
k \\
l-j
\end{array}\right) \cdot\left(\begin{array}{c}
u \\
j
\end{array}\right)} \cdot \sum_{\substack{\mathfrak{a} \in X(k+u): \\
\mathfrak{a} \sqcup\left(\mathfrak{s} \sqcup \mathfrak{t}^{\prime}\right)}} p_{\mathfrak{s}}^{(u)}(\mathfrak{a}) .
$$

Lemma 4.10 (Height Independence). Let $u \in[j, d-k]$. For any $\mathfrak{s} \in X(k), \mathfrak{s}^{\prime} \subseteq \mathfrak{s}$ and $\mathfrak{t}^{\prime} \in X(j)$ satisfying $\mathfrak{s}^{\prime} \sqcup \mathfrak{t}^{\prime} \in X(l)$ we have the following,

$$
\mathrm{S}_{k, l}^{(u, j)}\left(\mathfrak{s}, \mathfrak{s}^{\prime} \sqcup \mathfrak{t}^{\prime}\right)=\frac{1}{\left(\begin{array}{c}
k \\
l-j
\end{array}\right)\left(\begin{array}{c}
k+j \\
j
\end{array}\right)} \cdot \frac{\Pi_{k+j}\left(\mathfrak{s} \sqcup \mathfrak{t}^{\prime}\right)}{\Pi_{k}(\mathfrak{s})} .
$$

In particular, the choice of $u \in[j, d-k]$ does not affect the swap walk. 
Proof. We have,

$$
\begin{aligned}
\sum_{\mathfrak{a} \in X(k+u): \mathfrak{a} \supseteq \mathfrak{s} \sqcup \mathfrak{t}^{\prime}} p_{\mathfrak{s}}^{(k+u)}(\mathfrak{a}) & =\frac{1}{\left(\begin{array}{c}
k+u \\
u
\end{array}\right)} \cdot \frac{1}{\Pi_{k}(\mathfrak{s})} \cdot \sum_{\mathfrak{a} \in X(k+u): \mathfrak{a} \supseteq \mathfrak{s} \sqcup \mathfrak{t}} \Pi_{k+u}(\mathfrak{a}), \\
& =\frac{\left(\begin{array}{c}
k+u \\
u-j
\end{array}\right)}{\left(\begin{array}{c}
k+u \\
u
\end{array}\right)} \cdot \frac{\Pi_{k+j}\left(\mathfrak{s} \sqcup \mathfrak{t}^{\prime}\right)}{\Pi_{k}(\mathfrak{s})}
\end{aligned}
$$

where the first equality is due to Proposition 4.1 and the second is due to Proposition 2.4 together with the observation that $\mathfrak{s} \sqcup \mathfrak{t}^{\prime} \in X(k+j)$.

Thus, by Proposition 4.9 we get,

$$
\mathrm{S}_{k, l}^{(u, j)}(\mathfrak{s}, \mathfrak{t})=\frac{1}{\left(\begin{array}{c}
u \\
j
\end{array}\right) \cdot\left(\begin{array}{c}
k \\
l-j
\end{array}\right)} \frac{\left(\begin{array}{c}
k+u \\
u-j
\end{array}\right)}{\left(\begin{array}{c}
k+u \\
u
\end{array}\right)} \cdot \frac{\Pi_{k+j}\left(\mathfrak{s} \sqcup \mathfrak{t}^{\prime}\right)}{\prod_{k}(\mathfrak{s})} .
$$

We complete the proof by noting that,

$$
\frac{\left(\begin{array}{c}
k+u \\
u-j
\end{array}\right)}{\left(\begin{array}{c}
k+u \\
u
\end{array}\right)}=\frac{\left(\begin{array}{c}
u \\
j
\end{array}\right)}{\left(\begin{array}{c}
k+j \\
j
\end{array}\right)}
$$

and thus

$$
\mathrm{S}_{k, l}^{(u, j)}(\mathfrak{s}, \mathfrak{t})=\frac{1}{\left(\begin{array}{c}
k \\
l-j
\end{array}\right) \cdot\left(\begin{array}{c}
k+j \\
j
\end{array}\right)} \cdot \frac{\Pi_{k+j}\left(\mathfrak{s} \sqcup \mathfrak{t}^{\prime}\right)}{\Pi_{k}(\mathfrak{s})}
$$

which proves the formula.

Since the choice of $u$ does not affect the formula, we obtain Lemma 4.8.

\subsection{Canonical Walks in Terms of the Swap Walks}

We show that the canonical walks are given by an average of swap walks with respect to the hypergeometric distribution.

Lemma 4.11. Let $u, l, k, d \geq 0$ be given satisfying $l \leq k$ and $u \leq l$. Then, we have the following formula for the canonical $u$-walk on any $X(\leq d)$ satisfying $d \geq k+u$

$$
\mathrm{N}_{k, l}^{(u)}=\sum_{j=0}^{u} \frac{\left(\begin{array}{c}
u \\
j
\end{array}\right)\left(\begin{array}{c}
k \\
l-j
\end{array}\right)}{\left(\begin{array}{c}
k+u \\
l
\end{array}\right)} \cdot \mathrm{S}_{k, l}^{(j)}
$$

Proof. Suppose the canonical $u$-walk starting from $\mathfrak{s} \in X(k)$ picks $\mathfrak{s}^{\prime \prime} \in X(k+u)$ in the second step. Write $\mathcal{E}_{j}\left(\mathfrak{s}^{\prime \prime}\right)$ for the event that the random face $\mathfrak{s}^{\prime}$ the canonical $u$-walk picks in the descent step satisfies

$$
\left|\mathfrak{s}^{\prime} \backslash \mathfrak{s}\right|=j
$$

By elementary combinatorics,

$$
\underset{\mathfrak{s}^{\prime} \subseteq \mathfrak{s}^{\prime \prime}}{\mathbb{P}}\left[\mathcal{E}_{j}\left(\mathfrak{s}^{\prime \prime}\right) \mid \mathfrak{s}^{\prime \prime}\right]=\frac{\left(\begin{array}{c}
u \\
j
\end{array}\right)\left(\begin{array}{c}
k \\
l-j
\end{array}\right)}{\left(\begin{array}{c}
k+u \\
l
\end{array}\right)}
$$


where the draw of the probability is over the subsets $\mathfrak{s}^{\prime} \in X(l)$ of $\mathfrak{s}^{\prime \prime}$. Further, let $\mathfrak{t}_{j}^{\prime}$ be the random variable that stands for the face picked in the descent step of the $j$-swapping $u$-walk from $X(k)$ to $X(l)$.

By the definition of the $j$-swapping walk from $X(k)$ to $X(l)$, conditioning that the ascent step picks the same $\mathfrak{s}^{\prime \prime} \in X(k+u)$ we have

$$
\mathbb{P}\left[\mathfrak{t}_{j}^{\prime}=\mathfrak{t} \mid \mathfrak{s}^{\prime \prime}\right]=\mathbb{P}\left[\mathfrak{s}^{\prime}=\mathfrak{t} \mid \mathfrak{s}^{\prime \prime} \text { and } \mathcal{E}_{j}\left(\mathfrak{s}^{\prime \prime}\right)\right]
$$

Now, by the law of total probability we have

$$
\begin{aligned}
\mathbf{N}_{k, l}^{(u)}(\mathfrak{s}, \mathfrak{t})=\mathbb{P}\left[\mathbf{S}^{\prime}=\mathfrak{t}\right] & =\sum_{j=0}^{u} \sum_{\mathfrak{s}^{\prime \prime} \in X(k+u)} \mathbb{P}\left[\mathfrak{s}^{\prime \prime}\right] \cdot \mathbb{P}\left[\mathcal{E}_{j}\left(\mathfrak{s}^{\prime \prime}\right) \mid \mathfrak{s}^{\prime \prime}\right] \cdot \mathbb{P}\left[\mathfrak{s}^{\prime}=\mathfrak{t} \mid \mathfrak{s}^{\prime \prime} \text { and } \mathcal{E}_{j}\left(\mathfrak{s}^{\prime \prime}\right)\right] \\
& =\sum_{j=0}^{u} \frac{\left(\begin{array}{c}
u \\
j
\end{array}\right)\left(\begin{array}{c}
k \\
l-j
\end{array}\right)}{\left(\begin{array}{c}
k+u \\
l
\end{array}\right)} \cdot \underset{\mathfrak{s}^{\prime \prime} \supseteq \mathfrak{s}}{\mathbb{E}}\left[\mathbb{P}\left[\mathfrak{s}^{\prime}=\mathfrak{t} \mid \mathfrak{s}^{\prime \prime} \text { and } \mathcal{E}_{j}\left(\mathfrak{s}^{\prime \prime}\right)\right]\right] \\
& =\sum_{j=0}^{u} \frac{\left(\begin{array}{c}
u \\
j
\end{array}\right)\left(\begin{array}{c}
k+u \\
l-j
\end{array}\right)}{\left(\begin{array}{c}
k+u \\
l
\end{array}\right)} \cdot \underset{\mathfrak{s}^{\prime \prime} \supseteq \mathfrak{s}}{\mathbb{E}}\left[\mathbb{P}\left[\mathfrak{t}_{j}^{\prime}=\mathfrak{t} \mid \mathfrak{s}^{\prime \prime}\right]\right]
\end{aligned}
$$

where we used Equation (1) to get the last equality. Another application of the law of total probability gives us

$$
\underset{\mathfrak{s}^{\prime \prime} \supseteq \mathfrak{s}}{\mathbb{E}}\left[\mathbb{P}\left[\mathfrak{t}_{j}^{\prime}=\mathfrak{t} \mid \mathfrak{s}^{\prime \prime}\right]\right]=\mathbb{P}\left[\mathfrak{t}_{j}^{\prime}=\mathfrak{t}\right] .
$$

This allows us to write,

$$
\begin{aligned}
\mathbf{N}_{k, l}^{(u)}(\mathfrak{s}, \mathfrak{t}) & =\sum_{j=0}^{u} \frac{\left(\begin{array}{c}
u \\
j
\end{array}\right)\left(\begin{array}{c}
k \\
l-j
\end{array}\right)}{\left(\begin{array}{c}
k+u \\
l
\end{array}\right)} \cdot \mathbb{P}\left[\mathfrak{t}_{j}^{\prime}=\mathfrak{t}\right], \\
& =\sum_{j=0}^{u} \frac{\left(\begin{array}{c}
u \\
j
\end{array}\right)\left(\begin{array}{c}
k \\
l-j
\end{array}\right)}{\left(\begin{array}{c}
k+u \\
l
\end{array}\right)} \cdot \mathrm{S}_{k, l}^{(u, j)}(\mathfrak{s}, \mathfrak{t}),
\end{aligned}
$$

The statement follows using height independence, i.e. Lemma 4.8

\subsection{Inversion: Swap Walks in Terms of Canonical Walks}

We show how the swap walks can be obtained as a signed sum of canonical walks. This result follows from binomial inversion which we recall next.

Fact 4.12 (Binomial Inversion, [BS02]). Let $\left(a_{n}\right)_{n \geq 0},\left(b_{n}\right)_{n \geq 0}$ be arbitrary sequences. Suppose for all $n \geq 0$ we have,

$$
b_{n}=\sum_{j=0}^{n}\left(\begin{array}{l}
n \\
j
\end{array}\right) \cdot(-1)^{j} \cdot a_{j} .
$$

Then, we also have

$$
a_{n}=\sum_{j=0}^{n}\left(\begin{array}{l}
n \\
j
\end{array}\right) \cdot(-1)^{j} \cdot b_{j}
$$


Corollary 4.13. Let $k, l, d \geq 0$ be given parameters such that $k+l \leq d$ and $k \geq l$. For any simplicial complex $X(\leq d)$, one has the following formula for the $u$-swapping walk from $X(k)$ to $X(l)$ in terms of the canonical $j$-walks:

$$
\left(\begin{array}{c}
k \\
l-u
\end{array}\right) \mathrm{S}_{k, l}^{(u)}=\sum_{j=0}^{u}(-1)^{u-j} \cdot\left(\begin{array}{c}
k+j \\
l
\end{array}\right) \cdot\left(\begin{array}{c}
u \\
j
\end{array}\right) \cdot \mathrm{N}_{k, l}^{(j)}
$$

Proof. Fix faces $\mathfrak{s} \in X(k)$ and $\mathfrak{t} \in X(l)$ and set for all $j \in[0, u]$

$$
a_{j}:=\left(\begin{array}{c}
k \\
l-j
\end{array}\right) \cdot(-1)^{j} \cdot \mathrm{S}_{k, l}^{(j)}(\mathfrak{s}, \mathfrak{t}) \text {. }
$$

Notice that we have by Lemma 4.11

$$
\left(\begin{array}{c}
k+u \\
l
\end{array}\right) \cdot \mathrm{N}_{k, l}^{(u)}(\mathfrak{s}, \mathfrak{t})=\sum_{j=0}^{u}\left(\begin{array}{c}
u \\
j
\end{array}\right) \cdot(-1)^{j} \cdot a_{j}=\sum_{j=0}^{u}\left(\begin{array}{c}
u \\
j
\end{array}\right) \cdot\left(\begin{array}{c}
k \\
l-j
\end{array}\right) \cdot \mathrm{S}_{k, l}^{(j)}(\mathfrak{s}, \mathfrak{t}) .
$$

i.e. if we set

$$
b_{u}=\left(\begin{array}{c}
k+u \\
l
\end{array}\right) \cdot \mathrm{N}_{k, l}^{(u)}(\mathfrak{s}, \mathfrak{t})
$$

we can apply Fact 4.12 to obtain

$$
\begin{aligned}
\left(\begin{array}{c}
k \\
l-u
\end{array}\right) \cdot(-1)^{u} \cdot \mathrm{S}_{k, l}^{(u)}(\mathfrak{s}, \mathfrak{t}) & =a_{u} \\
& =\sum_{j=0}^{u}\left(\begin{array}{c}
u \\
j
\end{array}\right) \cdot(-1)^{j} \cdot b_{j} \\
& =\sum_{j=0}^{u}\left(\begin{array}{c}
u \\
j
\end{array}\right) \cdot\left(\begin{array}{c}
k+j \\
l
\end{array}\right) \cdot(-1)^{j} \cdot \mathrm{N}_{k, l}^{(j)}(\mathfrak{s}, \mathfrak{t}) .
\end{aligned}
$$

Dividing both sides of this equation by $(-1)^{u}$ yields the desired result.

\section{Spectral Analysis of Swap Walks}

Swap walks arise naturally in our $k$-CSPs approximation scheme on HDXs where the running time and the quality of approximation depend on the expansion of these walks. For this reason, we analyze the spectra of swap walks. We show that swap walks $S_{k, k}$ of $\gamma$-HDXs are indeed expanding for $\gamma$ sufficiently small. More precisely, the first main result of this section is the following.

Theorem 5.1 (Swap Walk Spectral Bound). Let $X(\leq d)$ be a $\gamma-H D X$ with $d \geq 2 k$. Then the second largest singular value $\sigma_{2}\left(S_{k, k}\right)$ of the swap operator $S_{k, k}$ is

$$
\sigma_{2}\left(\mathrm{~S}_{k, k}\right) \leq \gamma \cdot\left(2^{7} \cdot k^{4} \cdot 2^{3 k} \cdot k^{k}\right)
$$

Theorem 5.1 is enough for the analysis of our $k$-CSP approximation scheme when $k$ is a power of two. However, to analyze general $k$-CSPs on HDXs we need to understand the spectra of general swap walks $S_{k, l}$ where $k$ may differ from $l$. Therefore, we generalize the spectral analysis of $S_{k, k}$ above to $S_{k, l}$ obtaining Theorem 5.2, our second main result of this section. 
Theorem 5.2 (Rectangular Swap Walk Spectral Bound). Suppose $X(\leq d)$ is a $\gamma$-HDX with $d \geq k+l$ and $k \leq l$. Then the largest non-trivial singular value $\sigma_{2}\left(\mathrm{~S}_{k, l}\right)$ of the swap operator $\mathrm{S}_{k, l}$ is

$$
\sigma_{2}\left(\mathrm{~S}_{k, l}\right) \leq \sqrt{\gamma \cdot\left(2^{8} \cdot k^{2} \ell^{2} \cdot 2^{2 k+4 l} \cdot k^{k}\right)}
$$

\subsection{Square Swap Walks $S_{k, k}$}

We prove Theorem 5.1 by connecting the spectral structure of $S_{k, k}$ of general $\gamma$-HDXs to the well behaved case of complete simplicial complexes. To distinguish these two cases we denote by $S_{k, k}^{\Delta}$ the swap $S_{k, k}$ of complete complexes ${ }^{2}$. In fact, $S_{k, k}^{\Delta}$ is the random walk operator of the well known Kneser graph $K(n, k)$ (see Definition 5.3).

Definition 5.3 (Kneser Graph $K(n, k)$ [GM15]). The Kneser graph $K(n, k)$ is the graph $G=$ $(V, E)$ where $V=\left(\begin{array}{c}{[n]} \\ k\end{array}\right)$ and $E=\{\{\mathfrak{s}, \mathfrak{t}\} \mid \mathfrak{s} \cap \mathfrak{t}=\varnothing\}$.

Then at least for complete complexes we know that $S_{k, k}^{\Delta}$ is expanding. This is a direct consequence of Fact 5.4.

Fact 5.4 (Kneser Graph [GM15]). The singular values ${ }^{3}$ of the Kneser graph $K(n, k)$ are

$$
\left(\begin{array}{c}
n-k-i \\
k-i
\end{array}\right)
$$

for $i=0, \ldots, k$.

This means that $\sigma_{2}\left(\mathrm{~S}_{k, k}^{\Delta}\right)=O_{k}(1 / n)$ as shown in Claim 5.5.

Claim 5.5. Let $d \geq 2 k$ and $\Delta_{d}(n)$ be the complete complex. The second largest singular value $\sigma_{2}\left(\mathrm{~S}_{k, k}^{\Delta}\right)$ of the swap operator $\mathrm{S}_{k, k}^{\Delta}$ on $\Delta_{d}(n)$ is

$$
\sigma_{2}\left(\mathrm{~S}_{k, k}^{\Delta}\right)=\frac{k}{n-k}
$$

provided $n \geq M_{k}$ where $M_{k} \in \mathbb{N}$ only depends on $k$.

Proof. First note that for the complete complex $\Delta_{d}(n)$, the operator $\mathrm{S}_{k, k}^{\Delta}$ is the walk matrix of the Kneser graph $K(n, k)$. Since the degree of $K(n, k)$ is $\left(\begin{array}{c}n-k \\ k\end{array}\right)$, the result follows from Fact 5.4.

Therefore, if we could claim that $\sigma_{2}\left(\mathrm{~S}_{k, k}\right)$ of an arbitrary $\gamma$-HDX is close to $\sigma_{2}\left(\mathrm{~S}_{k, k}^{\Delta}\right)$ (provided $\gamma$ is sufficiently small), we would conclude that general $S_{k, k}$ walks are also expanding. A priori there is no reason why this claim should hold since a general $d$-sized $\gamma$-HDX may have much fewer hyperedges $\left(O_{d}(n)\right.$ versus $\left(\begin{array}{l}n \\ d\end{array}\right)$ in the complete $\left.\Delta_{d}(n)\right)$. Fortunately, it turns out that this claim is indeed true (up to $O_{k}(\gamma)$ errors).

To prove Theorem 5.1 we employ the beautiful expanding poset (EPoset) machinery of Dikstein et al. [DDFH18]. Before we delve into the full technical analysis, it might be

\footnotetext{
${ }^{2}$ The precise parameters of the complete complex $\Delta_{d}(n)$ where $S_{k, k}^{\Delta}$ lives will not be important. We only require that $S_{k, k}^{\Delta}$ is well defined in the sense that $d \geq 2 k$ and $n>d$.

${ }^{3}$ The precise eigenvalues are also well known, but singular values are enough in our analysis.
} 
instructive to see how Theorem 5.1 is obtained from understanding the quadratic form $\left\langle S_{k, k} f, f\right\rangle$ where $f \in C^{k}$.

First we informally recall the decomposition $C^{k}=\sum_{i=0}^{k} C_{i}^{k}$ from the EPoset machinery where $C_{i}^{k}$ can be thought of as the space of approximate eigenfunctions of degree $i$ of $C^{k}$ (the precise definitions are deferred to 5.2). In this decomposition, $C_{0}^{k}$ is defined as the space of constant functions of $C^{k}$.

We prove the stronger result that the $S_{k, k}$ operators of any $\gamma$-HDX has an an approximate spectrum that only depends on $k$ provided $\gamma$ is small enough. More precisely, we prove Lemma 5.6.

Lemma 5.6 (Swap Quadratic Form). Let $f=\sum_{i=0}^{k} f_{i}$ with $f_{i} \in C_{i}^{k}$. Suppose $X(\leq d)$ is a $\gamma$-HDX with $d \geq 2 k$. If $\gamma \leq \varepsilon\left(64 k^{k+4} 2^{3 k+1}\right)^{-1}$, then

$$
\left\langle\mathrm{S}_{k, k} f, f\right\rangle=\sum_{i=0}^{k} \lambda_{k}(i) \cdot\left\langle f_{i}, f_{i}\right\rangle \pm \varepsilon,
$$

where $\lambda_{k}(i)$ depends only on $k$ and $i$, i.e., $\lambda_{k}(i)$ is an approximate eigenvalue of $S_{k, k}$ associated to space $C_{i}^{k}$.

Remark 5.7. From Lemma 5.6, it might seem that we are done since there exist approximate eigenvalues $\lambda_{k}(i)$ that only depend on $k$ and $i$. However, giving an explicit expression for these approximate eigenvalues is tricky. For this reason, we rely on the expansion of Kneser graphs as will be clear later.

Towards showing Lemma 5.6, we introduce the notion of balanced operators which in particular captures canonical and swap walks and we show that the quadratic form expression of Lemma 5.6 is a particular case of a general result for $\langle\mathrm{B} f, f\rangle$ where $\mathrm{B}$ is a general balanced operator. A balanced operator in $C^{k}$ is any operator that can be obtained as linear combination of pure balanced operators, the later being operators that are a formal product of an equal number of up and down operators.

Lemma 5.8 (General Quadratic Form). Let $\varepsilon \in(0,1)$ and let $\mathcal{Y} \subseteq\left\{\mathrm{Y} \mid \mathrm{Y}: C^{k} \rightarrow C^{k}\right\}$ be a collection of formal operators that are product of an equal number of up and down walks (i.e., pure balanced operators) not exceeding $\ell$ walks. Let $\mathrm{B}=\sum_{\mathrm{Y} \in \mathcal{Y}} \alpha^{\mathrm{Y}} \mathrm{Y}$ where $\alpha^{\mathrm{Y}} \in \mathbb{R}$ and let $f=\sum_{i=0}^{k} f_{i}$ with $f_{i} \in C_{i}^{k}$. If $\gamma \leq \varepsilon\left(16 k^{k+2} \ell^{2} \sum_{Y \in \mathcal{Y}}\left|\alpha^{Y}\right|\right)^{-1}$, then

$$
\langle\mathrm{B} f, f\rangle=\sum_{i=0}^{k}\left(\sum_{\mathrm{Y} \in \mathcal{Y}} \alpha^{\mathrm{Y}} \lambda_{k}^{\mathrm{Y}}(i)\right) \cdot\left\langle f_{i}, f_{i}\right\rangle \pm \varepsilon,
$$

where $\lambda_{k}^{Y}(i)$ depends only on the operators appearing in the formal expression of $Y, i$ and $k$, i.e., $\lambda_{k}^{Y}(i)$ is the approximate eigenvalue of $Y$ associated to $C_{i}^{k}$.

Remark 5.9. Note that our result generalizes the analysis of [DDFH18] for expanding posets of HDXs which considered the particular case $\mathrm{B}=\mathrm{D}_{k+1} \mathrm{U}_{k}$. Moreover, their error term analysis treated all the parameters not depending on the number of vertices $n$ as constants. In this work we make the dependence on the parameters explicit since this dependence is important in understanding the limits of our $k$-CSPs approximation scheme on HDXs. The beautiful EPoset machinery [DDFH18] is instrumental in our analysis. 
Now, we are ready to prove Theorem 5.1. For convenience we restate it below.

Theorem 5.10 (Swap Walk Spectral Bound (restatement of Theorem 5.1)). Let $X(\leq d)$ be a $\gamma$-HDX with $d \geq 2 k$. For every $\sigma \in(0,1)$, if $\gamma \leq \sigma \cdot\left(64 k^{k+4} 2^{3 k+1}\right)^{-1}$, then the second largest singular value $\sigma_{2}\left(\mathrm{~S}_{k, k}\right)$ of the swap operator $\mathrm{S}_{k, k}$ is

$$
\sigma_{2}\left(S_{k, k}\right) \leq \sigma .
$$

Proof. First we show that for $i \in[k]$ the $i$-th approximate eigenvalue $\lambda_{k}(i)$ of the swap operator $S_{k, k}$ is actually zero. Note that for $i \in[k]$ the space $C_{i}^{k}$ is a non-trivial eigenspace (i.e., $C_{i}^{k}$ is not the space of constant functions). Let $S_{k, k}^{\Delta}$ be the swap operator of the complete complex $\Delta_{d}(n)$. On one hand Claim 5.5 gives

$$
\sigma_{2}\left(\mathrm{~S}_{k, k}^{\Delta}\right)=\max _{f \in C^{k}: f \perp 1,\|f\|=1}\left|\left\langle\mathrm{~S}_{k, k}^{\Delta} f, f\right\rangle\right|=O_{k}\left(\frac{1}{n}\right) .
$$

On the other hand since $\Delta_{d}(n)$ is a $\gamma^{\Delta}$-HDX where $\gamma^{\Delta}=O_{k}(1 / n)$, if $n$ is sufficiently large we have $\gamma^{\Delta} \leq \gamma$ and thus Lemma 5.8 can be applied to give

$$
\sigma_{2}\left(\mathrm{~S}_{k, k}^{\Delta}\right) \geq \max _{f_{i} \in C_{i}^{k}: i \in[k],\left\|f_{i}\right\|=1}\left|\left\langle\mathrm{~S}_{k, k}^{\Delta} f_{i}, f_{i}\right\rangle\right|=\left|\lambda_{k}(i)\right| \cdot\left\langle f_{i}, f_{i}\right\rangle \pm O_{k}\left(\frac{1}{n}\right) .
$$

Since $n$ is arbitrary and $\lambda_{k}(i)$ depends only on $k$ and $i$, we obtain $\lambda_{k}(i)=0$ as claimed. Now applying Lemma 5.8 to the swap operator $S_{k, k}$ of the $\gamma$-HDX $X(\leq d)$ yields

$$
\sigma_{2}\left(\mathrm{~S}_{k, k}\right)=\max _{f \in C^{k}: f \perp 1,\|f\|=1}\left|\left\langle\mathrm{~S}_{k, k} f, f\right\rangle\right| \leq \max _{i \in[k]}\left|\lambda_{k}(i)\right|+\sigma=\sigma,
$$

concluding the proof.

\subsection{Expanding Posets and Balanced Operators}

We state the definitions used in our technical proofs starting with $\gamma$-EPoset from [DDFH18].

Definition 5.11 ( $\gamma$-EPoset adapted from [DDFH18]). A complex $X(\leq d)$ with operators $\mathrm{U}_{0}, \ldots, \mathrm{U}_{d-1}$, $\mathrm{D}_{1}, \ldots, \mathrm{D}_{d}$ is said to be a $\gamma$-EPoset ${ }^{4}$ provided

$$
\left\|\mathrm{M}_{i}^{+}-\mathrm{U}_{i-1} \mathrm{D}_{i}\right\|_{\mathrm{op}} \leq \gamma,
$$

for every $i=1, \ldots, d-1$, where

$$
\mathrm{M}_{i}^{+}:=\frac{i+1}{i}\left(\mathrm{D}_{i+1} \mathrm{U}_{i}-\frac{1}{i+1} \mathrm{l}\right),
$$

i.e., $\mathrm{M}_{i}^{+}$is the non-lazy version of the random walk $\mathrm{N}_{i, i}^{(1)}=\mathrm{D}_{i+1} \mathrm{U}_{i}$.

Definition 5.11 can be directly used as an operational definition of high-dimension expansion as done in [DDFH18]. To us it is important that $\gamma$-HDXs are also $\gamma$-EPosets as established in Lemma 5.12. In fact, these two notions are known to be closely related.

\footnotetext{
${ }^{4}$ We tailor their general EPoset definition to HDXs. In fact, what they call $\gamma$-HDX we call $\gamma$-EPoset. Moreover, what they call $\gamma$-HD expander we call $\gamma$-HDX.
} 
Lemma 5.12 (From [DDFH18]). Let X be a d-sized simplicial complex.

- If $X$ is a $\gamma$-HDX, then $X$ is a $\gamma$-EPoset.

- If $X$ is a $\gamma$-EPoset, then $X$ is a $3 d \gamma-H D X$.

Naturally the complete complex $\Delta_{d}(n)$ is a $\gamma$-EPoset since it is a $\gamma$-HDX. Moreover, in this particular case $\gamma$ vanishes as $n$ grows.

Lemma 5.13 (From [DDFH18]). The complete complex $\Delta_{d}(n)$ is a $\gamma$-EPoset with $\gamma=O_{d}(1 / n)$.

\section{Harmonic Analysis on Simplicial Complexes}

The space $C^{k}$ defined in Section 2.2.2 can be decomposed into subspaces $C_{i}^{k}$ of functions of degree $i$ for $0 \leq i \leq k$ where

$$
C_{i}^{k}:=\left\{\mathrm{U}^{k-i} h_{i} \mid h_{i} \in H_{i}\right\}
$$

with $H_{i}:=\operatorname{ker}\left(D_{i}\right)$, and

$$
C_{0}^{k}:=\{f: X(k) \rightarrow \mathbb{R} \mid f \text { is constant }\} .
$$

More precisely, we have the following.

Lemma 5.14 (From [DDFH18]).

$$
C^{k}=\sum_{i=0}^{k} C_{i}^{k}
$$

Lemma 5.14 is proven in Appendix B as Lemma B.3.

For convenience set $\vec{\delta} \in \mathbb{R}^{d-1}$ such that $\delta_{i}=1 /(i+1)$ for $i \in[d-1]$. It will also be convenient to work with the following equivalent version of Eq. (2)

$$
\left\|\mathrm{D}_{i+1} \mathrm{U}_{i}-\left(1-\delta_{i}\right) \mathrm{U}_{i-1} \mathrm{D}_{i}-\delta_{i}\right\|_{\mathrm{op}} \leq \frac{i}{i+1} \gamma
$$

Towards our goal of understanding quadratic forms of swap operators we study the approximate spectrum of operators of the form $Y=Y_{\ell} \ldots Y_{1}$ where each $Y_{i}$ is either an up or down operator, namely, $Y$ is a generalized random walk of $\ell$ steps. We regard the expression $Y_{\ell} \ldots Y_{1}$ defining $Y$ as a formal product.

Definition 5.15 (Pure Balanced Operator). We call $\mathrm{Y}: C^{k} \rightarrow C^{k}$ a pure balanced operator if $\mathrm{Y}$ can be defined as product $\mathrm{Y}_{\ell} \ldots \mathrm{Y}_{1}{ }^{5}$ where each $\mathrm{Y}_{i}$ is either an up or down operator. When we say that the spectrum of $Y$ depends on $Y$ we mean that it depends on $k$ and on the formal expression $\mathrm{Y}_{\ell} \ldots \mathrm{Y}_{1}$ (i.e., pattern of up and down operators).

Remark 5.16. By definition canonical walks $\mathrm{N}_{k, k}^{(u)}$ are pure balanced operators.

Taking linear combinations of pure balanced operators leads to the notion of balanced operators.

\footnotetext{
${ }^{5}$ For the analysis it is convenient to order the indices appearing in $Y_{\ell} \ldots Y_{1}$ in decreasing order from left to right.
} 
Definition 5.17 (Balanced Operator). We call B: $C^{k} \rightarrow C^{k}$ a balanced operator provided there exists a set of pure balanced operators $\mathcal{Y}$ such that

$$
\mathrm{B}=\sum_{\mathrm{Y} \in \mathcal{Y}} \alpha^{\mathrm{Y}} \cdot \mathrm{Y}
$$

where $\alpha^{\mathrm{Y}} \in \mathbb{R}$.

Remark 5.18. Corollary 4.13 establishes that $\mathrm{S}_{k, k}^{(u)}$ are balanced operators. In particular, $\mathrm{S}_{k, k}$ is a balanced operator.

It turns out that at a more crude level the behavior of $\mathrm{Y}$ is governed by how the number of up operators compares to the number of down operators. For this reason, it is convenient to define $\mathcal{U}(Y)=\left\{Y_{i} \mid Y_{i}\right.$ is an up operator $\}$ and $\mathcal{D}(Y)=\left\{Y_{i} \mid Y_{i}\right.$ is a down operator $\}$ where $\mathrm{Y}$ is a pure balanced operator. When $\mathrm{Y}$ is clear in the context we use $\mathcal{U}=\mathcal{U}(\mathrm{Y})$ and $\mathcal{D}=\mathcal{D}(\mathrm{Y})$.

Henceforth we assume $h_{i} \in H_{i}=\operatorname{ker}\left(\mathrm{D}_{i}\right), f_{i} \in C_{i}^{k}$ and $g \in C^{k}$. This convention will make the statements of the technical results of Section 5.3 cleaner.

\subsection{Quadratic Forms over Balanced Operators}

Now we establish all the technical results leading to and including the analysis of quadratic forms over balanced operators. By considering this general class of operators our analysis generalizes the analysis given in [DDFH18]. At the same time we refine their error terms analysis by making the dependence on the EPoset parameters explicit. Recall that an explicit dependence on these parameters is important in understanding the limits of our $k$-CSP approximation scheme.

Lemma 5.19 (General Quadratic Form (restatement of Lemma 5.8)). Let $\varepsilon \in(0,1)$ and let $\mathcal{Y} \subseteq\left\{\mathrm{Y} \mid \mathrm{Y}: C^{k} \rightarrow C^{k}\right\}$ be a collection of formal operators that are product of an equal number of up and down walks (i.e., pure balanced operators) not exceeding $\ell$ walks. Let $\mathrm{B}=\sum_{\mathrm{Y} \in \mathcal{Y}} \alpha^{\mathrm{Y}} \mathrm{Y}$ where $\alpha^{\mathrm{Y}} \in \mathbb{R}$ and let $f=\sum_{i=0}^{k} f_{i}$ with $f_{i} \in C_{i}^{k}$. If $\gamma \leq \varepsilon\left(16 k^{k+2} \ell^{2} \sum_{\mathrm{Y} \in \mathcal{Y}}\left|\alpha^{\mathrm{Y}}\right|\right)^{-1}$, then

$$
\langle\mathrm{B} f, f\rangle=\sum_{i=0}^{k}\left(\sum_{\mathrm{Y} \in \mathcal{Y}} \alpha^{\mathrm{Y}} \lambda_{k}^{\mathrm{Y}}(i)\right) \cdot\left\langle f_{i}, f_{i}\right\rangle \pm \varepsilon,
$$

where $\lambda_{k}^{Y}(i)$ depends only on the operators appearing in the formal expression of $\mathrm{Y}, i$ and $k$, i.e., $\lambda_{k}^{Y}(i)$ is the approximate eigenvalue of $Y$ associated to $C_{i}^{k}$.

Since swap walks are balanced operators, we will deduced the following (as proven later).

Lemma 5.20 (Swap Quadratic Form (restatement of Lemma 5.6)). Let $f=\sum_{i=0}^{k} f_{i}$ with $f_{i} \in C_{i}^{k}$. Suppose $X(\leq d)$ is a $\gamma$-HDX with $d \geq 2 k$. If $\gamma \leq \varepsilon\left(64 k^{k+4} 2^{3 k+1}\right)^{-1}$, then

$$
\left\langle\mathrm{S}_{k, k} f, f\right\rangle=\sum_{i=0}^{k} \lambda_{k}(i) \cdot\left\langle f_{i}, f_{i}\right\rangle \pm \varepsilon,
$$

where $\lambda_{k}(i)$ depends on only on $k$ an $i$, i.e., $\lambda_{k}(i)$ is an approximate eigenvalue of $S_{k, k}$ associated to space $C_{i}^{k}$. 
The next result, Lemma 5.21, (implicit in [DDFH18]) will be key in establishing that the spectral structure of $\gamma$-EPosets is fully determined by the parameters in $\vec{\delta}$ provided $\gamma$ is small enough. Note that the Eposet Definition 5.11 provides a "calculus" for rearranging a single pair of up and down DU. The next result treats the more general case of DU $\cdots U$.

Lemma 5.21 (Structure Lemma). Suppose $|\mathcal{D}|=1$. Let $\mathrm{Y}_{c} \in \mathcal{D}$ be the unique down operator in $\mathrm{Y}_{\ell} \ldots \mathrm{Y}_{1}$. If $\|\mathrm{A}\|_{\mathrm{op}} \leq 1$, then

$$
\left\langle\mathrm{AY}_{\ell} \ldots \mathrm{Y}_{1} h_{i}, g\right\rangle= \begin{cases}0 & \text { if } \ell=1 \text { or } c=1 \\ Q_{c, i}(\vec{\delta}) \cdot\left\langle\mathrm{AU}^{\ell-2} h_{i}, g\right\rangle \pm(c-1) \cdot \gamma\left\|h_{i}\right\|\|g\| & \text { otherwise, }\end{cases}
$$

where $Q_{c, i}$ is a polynomial in the variables $\vec{\delta}$ depending on $c, i$ such that $Q_{c, i}(\vec{\delta}) \leq 1$.

Proof. We induct on $(\ell, c)$. If $\ell=1$ or $c=1$, we have $\mathrm{Y}_{1} h_{i}=\mathrm{D}_{i} h_{i}=0$ so the result trivially holds. Otherwise, we have $\mathrm{Y}_{c} \mathrm{Y}_{c-1}=\mathrm{D}_{j+1} \mathrm{U}_{j}$ where $j=i+c-2$. Then

$$
\left\langle A Y_{\ell} \ldots Y_{c+1}\left(Y_{c} Y_{c-1}\right) Y_{c-2} \ldots Y_{1} h_{i}, g\right\rangle
$$

becomes

$$
\begin{aligned}
& \left(1-\delta_{j}\right) \cdot\left\langle\mathrm{AY}_{\ell} \ldots \mathrm{Y}_{c+1} \mathrm{U}_{j-1} \mathrm{D}_{j} \mathrm{Y}_{c-2} \ldots \mathrm{Y}_{1} h_{i}, g\right\rangle+\delta_{j} \cdot\left\langle\mathrm{AY}_{\ell} \ldots \mathrm{Y}_{c+1} \mathrm{Y}_{c-2} \ldots \mathrm{Y}_{1} h_{i}, g\right\rangle \pm \gamma\left\|h_{i}\right\|\|g\| \\
& =\left(1-\delta_{j}\right) \cdot\left\langle\mathrm{AY}_{1} \ldots \mathrm{Y}_{c-1} \mathrm{U}_{j-1} \mathrm{D}_{j} \mathrm{Y}_{c+2} \ldots \mathrm{Y}_{\ell} h_{i}, g\right\rangle+\delta_{j} \cdot\left\langle\mathrm{AU}^{\ell-2} h_{i}, g\right\rangle \pm \gamma\left\|h_{i}\right\|\|g\| \\
& =\left(1-\delta_{j}\right) \cdot Q_{c-1, i}(\vec{\delta}) \cdot\left\langle\mathrm{AU}^{\ell-2} h_{i}, g\right\rangle \pm\left(1-\delta_{j}\right) \cdot(c-2) \gamma\left\|h_{i}\right\|\|g\|+\delta_{j} \cdot\left\langle\mathrm{AU}^{\ell-2} h_{i}, g\right\rangle \pm \gamma\left\|h_{i}\right\|\|g\| \\
& =Q_{c, i}(\vec{\delta}) \cdot\left\langle\mathrm{AU}^{\ell-2} h_{i}, g\right\rangle \pm(c-1) \cdot \gamma\left\|h_{i}\right\|\|g\| .
\end{aligned}
$$

With Lemma 5.21 we are close to recover the approximate spectrum of $\mathrm{D}_{k+1} \mathrm{U}_{k}$ from [DDFH18]. However, in our application we will need to analyze more general operators, namely, pure balanced and balanced operators.

Lemma 5.22 (Refinement of [DDFH18]). If $\|\mathrm{A}\|_{\mathrm{op}} \leq 1$, then

$$
\left\langle\mathrm{AD}_{k+1} \mathrm{U}_{k} f_{i}, g\right\rangle=\lambda_{i} \cdot\left\langle\mathrm{A} f_{i}, g\right\rangle \pm(k-i+1) \cdot \gamma\left\|h_{i}\right\|\|g\|,
$$

where $\lambda_{i}=Q_{k-i+2, i}(\vec{\delta})$.

Proof. Recall that $f_{i}=\mathrm{U}^{k-i} h_{i}$ where $h_{i} \in \operatorname{ker}\left(\mathrm{D}_{i}\right)$. Set $\mathrm{Y}=\mathrm{D}_{k+1} \mathrm{U}_{k} \mathrm{U}^{k-i}$. Lemma 5.21 yields

$$
\left\langle\mathrm{AD}_{k+1} \mathrm{U}_{k} f_{i}, g\right\rangle=\lambda_{i} \cdot\left\langle\mathrm{A} f_{i}, g\right\rangle \pm(k-i+1) \cdot \gamma\left\|h_{i}\right\|\|g\|,
$$

where $\lambda_{i}=Q_{k-i+2, i}(\vec{\delta})$.

Then powers of the operator $D_{k+1} U_{k}$ behave as expected.

Lemma 5.23 (Exponentiation Lemma).

$$
\left\langle\left(\mathrm{D}_{k+1} \mathrm{U}_{k}\right)^{s} f_{i}, f_{i}\right\rangle=\lambda_{i}^{s} \cdot\left\|f_{i}\right\|^{2} \pm s \cdot(k-i+1) \cdot \gamma\left\|h_{i}\right\|\left\|f_{i}\right\|,
$$

where $\lambda_{i}$ is given in Lemma 5.22 . 
Proof. Follows immediately from the foregoing and the fact that $\left\|\mathrm{D}_{k+1} \mathrm{U}_{k}\right\|_{\mathrm{op}}=1$.

In case $|\mathcal{D}|>|\mathcal{U}|, \mathrm{Y}: C^{i} \rightarrow C^{j}$ is an operator whose kernel approximately contains $\operatorname{ker}\left(D_{i}\right)$ as the following lemma makes precise.

Lemma 5.24 (Refinement of [DDFH18]). If $|\mathcal{D}|>|\mathcal{U}|$ and $h_{i} \in \operatorname{ker}\left(\mathrm{D}_{i}\right)$, then

$$
\left\langle\mathrm{AY}_{\ell} \ldots \mathrm{Y}_{1} h_{i}, g\right\rangle= \pm \ell^{2} \cdot \gamma\left\|h_{i}\right\|\|g\|
$$

provided $\|\mathrm{A}\|_{\mathrm{op}} \leq 1$

Proof. Let $c \in[\ell]$ be the smallest index for which $Y_{c}$ is a down operator. Observe that $c<\ell / 2$ since $|\mathcal{D}|>|\mathcal{U}|$. We induct on $m=|\mathcal{D}|$. If $c=1$, then $\left\langle\mathrm{AD}_{i} h_{i}, g\right\rangle=0$. Hence assume $c, m>1$ implying $\mathrm{Y}_{c} \mathrm{Y}_{c-1}=\mathrm{D}_{i+c} \mathrm{U}_{i+c-1}$. Applying Lemma 5.21 we obtain

$$
\begin{aligned}
\left\langle\mathrm{AY}_{\ell} \ldots \mathrm{Y}_{1} h_{i}, g\right\rangle & =\left\langle\left(\mathrm{AY}_{\ell} \ldots \mathrm{Y}_{c+1}\right) \mathrm{DUU}^{c-2} h_{i}, g\right\rangle \\
& =Q_{c, i}(\vec{\delta}) \cdot\left\langle\left(\mathrm{AY}_{\ell} \ldots \mathrm{Y}_{c+1}\right) \mathrm{U}^{c-2} h_{i}, g\right\rangle \pm \frac{\ell}{2} \cdot \gamma\left\|h_{i}\right\|\|g\| \\
& = \pm Q_{c, i}(\vec{\delta}) \cdot(\ell-2)^{2} \cdot \gamma\left\|h_{i}\right\|\|g\| \pm \frac{\ell}{2} \cdot \gamma\left\|h_{i}\right\|\|g\| \quad \text { (Induction) } \\
& = \pm \ell^{2} \cdot \gamma\left\|h_{i}\right\|\|g\|,
\end{aligned}
$$

where in the last derivation we used $Q_{c, i}(\vec{\delta}) \leq 1$.

We turn to an important particular case of $|\mathcal{D}|=|\mathcal{U}|$, namely, the canonical walks. We show that $\mathrm{N}_{k, k}^{(u)}$ is approximately a polynomial in the operator $\mathrm{D}_{k+1} \mathrm{U}_{k}$. As a warm up consider the case $\mathrm{N}_{k, k}^{(2)}=\mathrm{D}_{k+1} \mathrm{D}_{k+2} \mathrm{U}_{k+1} \mathrm{U}_{k}$. Using the Eq. (3), we get

$$
\begin{aligned}
\mathrm{N}_{k, k}^{(2)} & \approx\left(1-\delta_{k+1}\right) \cdot \mathrm{D}_{k+1} \mathrm{U}_{k} \mathrm{D}_{k+1} \mathrm{U}_{k}+\delta_{k+1} \cdot \mathrm{D}_{k+1} \mathrm{U}_{k} \\
& =\left(1-\delta_{k+1}\right) \cdot\left(\mathrm{D}_{k+1} \mathrm{U}_{k}\right)^{2}+\delta_{k+1} \cdot \mathrm{D}_{k+1} \mathrm{U}_{k} .
\end{aligned}
$$

Inspecting this polynomial more carefully we see that that its coefficients form a probability distribution. This property holds in general as the following Lemma 5.25 shows. This gives an alternative (approximate) random walk interpretation of $\mathrm{N}_{k, k}^{(u)}$ as the walk that first selects the power $s$ according to the distribution encoded in the polynomial and then moves according to $\left(\mathrm{D}_{k+1} \mathrm{U}_{k}\right)^{S}$.

Lemma 5.25 (Canonical Polynomials). For $k, u \geq 0$ there exists a degree $u$ univariate polynomial $F_{u, k, \vec{\delta}}^{N}$ depending only on $u, k, \vec{\delta}$ such that

$$
\left\|\mathrm{N}_{k, k}^{(u)}-F_{u, k, \vec{\delta}}^{N}\left(\mathrm{D}_{k+1} \mathrm{U}_{k}\right)\right\|_{\mathrm{op}} \leq(u-1)^{2} \cdot \gamma
$$

Moreover, the coefficients of this polynomial form a probability distribution, i.e., $F_{u, k, \vec{\delta}}^{N}(x)=\sum_{i=0}^{u} c_{i} x^{i}$ where $\sum_{i=0}^{u} c_{i}=1$ and $c_{i} \geq 0$ for $i=0, \ldots, u$. 
Proof. For $u=0, \mathrm{~N}_{k, k}^{(0)}=\mathrm{I}$ and the lemma trivially follows. Similarly, if $u=1, \mathrm{~N}_{k, k}^{(1)}=$ $\mathrm{D}_{k+1} \mathrm{U}_{k}$. Now suppose $u \geq 2$. Set $\mathrm{Y}=\mathrm{N}_{k, k}^{(u)}$, i.e.,

$$
\mathrm{Y}=\mathrm{D}_{k+1} \ldots\left(\mathrm{D}_{k+u} \mathrm{U}_{k+u-1}\right) \ldots \mathrm{U}_{k}
$$

For convenience let $j=k+u-1$. Using the Eq. (3) we can replace $\mathrm{D}_{j+1} \mathrm{U}_{j}$ in $\mathrm{Y}$ by $(1-$ $\left.\delta_{j}\right) \mathrm{U}_{j-1} \mathrm{D}_{j}+\delta_{j} \mathrm{I}$ incurring an error of $\gamma$ (in spectral norm) and yielding

$$
\mathrm{Y} \approx\left(1-\delta_{j}\right) \cdot \mathrm{Y}^{\prime}+\delta_{j} \cdot \mathrm{N}_{k, k}^{(u-1)}
$$

where $Y^{\prime}$ was obtained from $Y$ by moving the rightmost occurence of a down operator (in this case $\mathrm{D}_{j+1}$ ) one position to right. We continue this process of moving the rightmost occurrence of a down operator until the resulting operator is up to $(u-1) \cdot \gamma$ error

$$
\alpha \cdot \mathrm{N}_{k, k}^{(u-1)}\left(\mathrm{D}_{k+1} \mathrm{U}_{k}\right)+\beta \cdot \mathrm{N}_{k, k}^{(u-1)},
$$

where $\alpha=\prod_{i=k+1}^{j}\left(1-\delta_{i}\right)$ and $\beta=\sum_{i=k+1}^{j} \delta_{i} \prod_{i=k+1}^{j}\left(1-\delta_{i}\right)$. Since $\delta_{i}=\delta_{i}>0, \alpha, \beta$ are non negative and form a probability distribution. Now the result follows from the induction hypothesis applied to $\mathrm{N}_{k, k}^{(u-1)}$.

Remark 5.26. Having a polynomial expression $F_{u, k, \vec{\delta}}^{N}\left(D_{k+1} \mathrm{U}_{k}\right) \approx \mathrm{N}_{k, k}^{(u)}$ and knowing that $\mathrm{S}_{k, k}$ can be written as linear combination of canonical walks, we could deduce that $S_{k, k}$ is also approximately a polynomial in $\mathrm{D}_{k+1} \mathrm{U}_{k}$. Using an error refined version of the Lemma 5.23 (showing that exponentiation of $\mathrm{D}_{k+1} \mathrm{U}_{k}$ behaves naturally), we could deduce the approximate spectrum of $\mathrm{S}_{k, k}$. We avoid this approach since it analysis introduces unnecessary error terms and we can understand quadratic forms of pure balanced operators directly.

Remark 5.27. The canonical polynomial $F_{u, k, \vec{\delta}}^{N}\left(\mathrm{D}_{k+1} \mathrm{U}_{k}\right)$ is used later in the error analysis that relates the norms $\left\|h_{i}\right\|$ and $\left\|f_{i}\right\|$ (Lemma 5.30).

Now we consider $Y$ where $|\mathcal{D}|=|\mathcal{U}|$ in full generality. We show how the quadratic form of $Y$ behaves in terms of the approximate eigenspace decomposition $C^{k}=\sum_{i=0}^{k} C_{i}^{k}$.

Lemma 5.28 (Pure Balanced Walks). Suppose $\mathrm{Y}=\mathrm{Y}_{\ell} \ldots \mathrm{Y}_{1}$ is a product of an equal number of up and down operators, i.e., $|\mathcal{D}|=|\mathcal{U}|$. Then for $f_{i} \in C_{i}^{k}$

$$
\left\langle Y f_{i}, f_{i}\right\rangle=\lambda_{k, i}^{Y} \cdot\left\langle f_{i}, f_{i}\right\rangle \pm \gamma \cdot\left(\ell^{2}+\ell(k-i-1)\right)\left\|h_{i}\right\|\left\|f_{i}\right\|,
$$

where $\lambda_{k, i}^{\mathrm{Y}}$ is an approximate eigenvalue depending only on $\mathrm{Y}, k$ and $i$.

Proof. We induct on even $\ell$. For $\ell=0$, the result trivially follows so assume $\ell \geq 2$. Let $c \in[\ell]$ be the smallest index of a down operator. Set $A=Y_{\ell} \ldots Y_{c+1}$ and let $Y^{\prime}=Y_{c} \ldots Y_{1}=$ DU ... U. Observe that

$$
\left\langle\mathrm{AY}^{\prime} f_{i}, f_{i}\right\rangle=\left\langle\mathrm{ADU}^{\mathrm{c}-1+k-i} h_{i}, f_{i}\right\rangle .
$$

Applying Lemma 5.21 to the RHS above gives

$$
\left\langle\mathrm{ADU}^{c-1+k-i} h_{i}, f_{i}\right\rangle=Q_{c-1+k-i, i}(\vec{\delta}) \cdot\left\langle\mathrm{AU}^{c-2} f_{i}, f_{i}\right\rangle \pm(c+k-i-2) \cdot \gamma\left\|h_{i}\right\|\left\|f_{i}\right\| .
$$


Applying the induction hypothesis to $Y^{\prime \prime}=A U^{c-2}$ in the above RHS yields

$$
\begin{aligned}
& Q_{c-1+k-i, i}(\vec{\delta}) \cdot \lambda_{k, i}^{Y \prime \prime}\left\langle f_{i}, f_{i}\right\rangle \\
& \pm Q_{c-1+k-i, i}(\vec{\delta}) \cdot \gamma \cdot\left((\ell-1)^{2}+(\ell-1)(k-i-1)\right)\left\|h_{i}\right\|\left\|f_{i}\right\| \\
& \pm(c+k-i-2) \cdot \gamma\left\|h_{i}\right\|\left\|f_{i}\right\| \\
& =\lambda_{k, i}^{Y} \cdot\left\langle f_{i}, f_{i}\right\rangle \pm \gamma \cdot\left(\ell^{2}+\ell(k-i-1)\right)\left\|h_{i}\right\|\left\|f_{i}\right\|,
\end{aligned}
$$

where $\lambda_{k, i}^{\mathrm{Y}}=Q_{c-1+k-i, i}(\vec{\delta}) \cdot \lambda_{k, i}^{\mathrm{Y}^{\prime \prime}}$ and the last equality follows from $Q_{c-1+k-i, i}(\vec{\delta}) \leq 1$ and $c \leq \ell$.

To understand all errors in the analysis in Lemma 5.28 we need to derive the approximate orthogonality of $f_{i}$ and $f_{j}$ for $i \neq j$ from [DDFH18] in more detail. We start with the following bound in terms of $h_{i}, h_{j}$.

Lemma 5.29 (Refinement of [DDFH18]). For $i \neq j$,

$$
\left\langle f_{i}, f_{j}\right\rangle= \pm(2 k-i-j)^{2} \cdot \gamma\left\|h_{i}\right\|\left\|h_{j}\right\| \text {. }
$$

Proof. Recall that $f_{i}=\mathrm{U}^{k-i} h_{i}, f_{j}=\mathrm{U}^{k-j} h_{j}$ where $h_{i} \in \operatorname{ker}\left(\mathrm{D}_{i}\right), h_{j} \in \operatorname{ker}\left(\mathrm{D}_{j}\right)$. Without loss of generality suppose $i>j$. We have

$$
\left\langle\mathrm{U}^{k-i} h_{i}, \mathrm{U}^{k-j} h_{j}\right\rangle=\left\langle\mathrm{D}^{k-j} \mathrm{U}^{k-i} h_{i}, h_{j}\right\rangle
$$

Since $k-j>k-i$, the result follows from Lemma 5.24.

To give a bound for Lemma 5.29 only in terms of the eigenfunction norms $\left\|f_{i}\right\|$ and not in terms of $\left\|h_{i}\right\|$, we need to understand how the norms of $h_{i}$ and $f_{i}$ are related.

Lemma 5.30 (Refinement of [DDFH18]). Let $\eta_{k, i}=(k-i)^{2}+1$ and let $\beta_{i}=\sqrt{\left|F_{k-i, i, \vec{\delta}}^{N}\left(\delta_{i}\right) \pm \gamma \cdot \eta_{k, i}\right|}$ where $F_{k-i, k, \vec{\delta}}^{N}$ is a canonical polynomial of degree $k-i$ from Lemma 5.25. Then

$$
\left\langle f_{i}, f_{i}\right\rangle=\beta_{i}^{2} \cdot\left\langle h_{i}, h_{i}\right\rangle
$$

Let $\theta_{k, i}=(i+1)^{k-i}$. Furthermore, if $\gamma \leq 1 /\left(2 \cdot \eta_{k, i} \cdot \theta_{k, i}\right)$, then $\beta_{i} \geq \frac{1}{2 \theta_{k, i}}$.

Proof. Recall that $f_{i}=\mathrm{U}^{k-i} h_{i}$ where $h_{i} \in \operatorname{ker}\left(\mathrm{D}_{i}\right)$. For $i=k$ the result trivially follows so assume $k>i$. First consider the case $k=i+1$. We have

$$
\left\langle\mathrm{U}_{i} h_{i}, \mathrm{U}_{i} h_{i}\right\rangle=\left\langle\mathrm{D}_{i+1} \mathrm{U}_{i} h_{i}, h_{i}\right\rangle=\delta_{i} \cdot\left\langle h_{i}, h_{i}\right\rangle \pm \gamma \cdot\left\langle h_{i}, h_{i}\right\rangle
$$

For general $k>i$ we have

$$
\left\langle\mathrm{U}^{k-i} h_{i}, \mathrm{U}^{k-i} h_{i}\right\rangle=\left\langle\mathrm{D}^{k-i} \mathrm{U}^{k-i} h_{i}, h_{i}\right\rangle .
$$

Applying Lemma 5.25 to $\mathrm{D}^{k-i} \mathrm{U}^{k-i}$ yields

$$
\left\langle\mathrm{D}^{k-i} \mathrm{U}^{k-i} h_{i}, h_{i}\right\rangle=\left\langle F_{k-i, i, \vec{\delta}}^{N}\left(\mathrm{D}_{i+1} \mathrm{U}_{i}\right) h_{i}, h_{i}\right\rangle \pm \gamma \cdot(k-i-1)^{2} .
$$


Combining Eq. (4) and Lemma 5.23 gives

$$
\left\langle F_{k-i, i, \vec{\delta}}^{N}\left(\mathrm{D}_{i+1} \mathrm{U}_{i}\right) h_{i}, h_{i}\right\rangle \pm \gamma \cdot(k-i-1)^{2}=\left\langle F_{k-i, i, \vec{\delta}}^{N}\left(\delta_{i}\right) h_{i}, h_{i}\right\rangle \pm \gamma \cdot\left((k-i)^{2}+1\right) .
$$

Since $F_{k-i, i, \vec{\delta}}^{N}(x)=\sum_{i=0}^{k-i} c_{i} x^{i}$ where the coefficients $c_{i}$ form a probability distribution, we get

$$
F_{k-i, i, \vec{\delta}}^{N}\left(\delta_{i}\right) \geq \delta_{i}^{k-i}=\left(\frac{1}{i+1}\right)^{k-i}
$$

Now, we can state the approximate orthogonality Lemma 5.31 in terms of the eigenfunction norms.

Lemma 5.31 (Approximate Orthogonality (refinement of [DDFH18])). Let $\eta_{k, s}, \theta_{k, s}, \beta_{s}$ for $s \in\{i, j\}$ be given as in Lemma 5.30. If $i \neq j$ and $\beta_{i}, \beta_{j}>0$, then

$$
\left\langle f_{i}, f_{j}\right\rangle= \pm \frac{\gamma \cdot(2 k-i-j)^{2}}{\beta_{i} \beta_{j}}\left\|f_{i}\right\|\left\|f_{j}\right\| \text {. }
$$

Furthermore, if $\gamma \leq \min \left(1 /\left(2 \cdot \eta_{k, i} \cdot \theta_{k, i}\right), 1 /\left(2 \cdot \eta_{k, j} \cdot \theta_{k, j}\right)\right)$, then $\beta_{i}, \beta_{j}>0$ and

$$
\left\langle f_{i}, f_{j}\right\rangle= \pm \gamma \cdot \theta_{k, i} \cdot \theta_{k, j} \cdot(2 k-i-j)^{2}\left\|f_{i}\right\|\left\|f_{j}\right\| \text {. }
$$

Proof. Follows directly from Lemma 5.30.

We generalize the quadratic form of Lemma 5.28 to linear combinations of general pure balanced operators $Y$, namely, to balanced operators.

Lemma 5.32 (General Quadratic Form (restatement of Lemma 5.8)). Let $\varepsilon \in(0,1)$ and let $\mathcal{Y} \subseteq\left\{\mathrm{Y} \mid \mathrm{Y}: C^{k} \rightarrow C^{k}\right\}$ be a collection of formal operators that are product of an equal number of up and down walks (i.e., pure balanced operators) not exceeding $\ell$ walks. Let $\mathrm{B}=\sum_{\mathrm{Y} \in \mathcal{Y}} \alpha^{\mathrm{Y}} \mathrm{Y}$ where $\alpha^{\mathrm{Y}} \in \mathbb{R}$ and let $f=\sum_{i=0}^{k} f_{i}$ with $f_{i} \in C_{i}^{k}$. If $\gamma \leq \varepsilon\left(16 k^{k+2} \ell^{2} \sum \mathrm{Y} \in \mathcal{Y}\left|\alpha^{\mathrm{Y}}\right|\right)^{-1}$, then

$$
\langle\mathrm{B} f, f\rangle=\sum_{i=0}^{k}\left(\sum_{\mathrm{Y} \in \mathcal{Y}} \alpha^{\mathrm{Y}} \lambda_{k}^{\mathrm{Y}}(i)\right) \cdot\left\langle f_{i}, f_{i}\right\rangle \pm \varepsilon,
$$

where $\lambda_{k}^{Y}(i)$ depends only on the operators appearing in the formal expression of $\mathrm{Y}, i$ and $k$, i.e., $\lambda_{k}^{Y}(i)$ is the approximate eigenvalue of $Y$ associated to $C_{i}^{k}$.

Proof. Using Lemma 5.28 and the assumption on $\gamma$ gives

$$
\begin{aligned}
\langle\mathrm{B} f, f\rangle= & \sum_{i=0}^{k} \sum_{\mathrm{Y} \in \mathcal{Y}} \alpha^{\mathrm{Y}} \lambda_{k}^{\mathrm{Y}}(i) \cdot\left\langle f_{i}, f_{i}\right\rangle \\
& \quad+\sum_{i \neq j} \sum_{\mathrm{Y} \in \mathcal{Y}}\left(\alpha^{\mathrm{Y}} \lambda_{k}^{\mathrm{Y}}(i) \cdot\left\langle f_{i}, f_{j}\right\rangle \pm \gamma \cdot \alpha^{\mathrm{Y}}\left(\ell^{2}+\ell(k-i-1)\right)\left\langle h_{i}, f_{j}\right\rangle\right) \\
= & \sum_{i=0}^{k} \sum_{\mathrm{Y} \in \mathcal{Y}} \alpha^{\mathrm{Y}} \lambda_{k}^{\mathrm{Y}}(i) \cdot\left\langle f_{i}, f_{i}\right\rangle+\sum_{i \neq j} \sum_{\mathrm{Y} \in \mathcal{Y}} \alpha^{\mathrm{Y}} \lambda_{k}^{\mathrm{Y}}(i) \cdot\left\langle f_{i}, f_{j}\right\rangle \pm \frac{\varepsilon}{2} .
\end{aligned}
$$

Next we use Lemma 5.31 to bound the second double summation and conclude the proof. 
We instantiate Lemma 5.31 for swap walks with their specific parameters. First, we introduce some notation. Using Corollary 4.13, we have

$$
\mathrm{S}_{k, k}=\sum_{j=0}^{k}(-1)^{k-j} \cdot\left(\begin{array}{c}
k+j \\
k
\end{array}\right) \cdot\left(\begin{array}{l}
k \\
j
\end{array}\right) \cdot \mathrm{N}_{k, k}^{(j)}=\sum_{j=0}^{k} \alpha_{j} \cdot \mathrm{N}_{k, k^{\prime}}^{(j)}
$$

where $\alpha_{j}=(-1)^{k-j} \cdot\left(\begin{array}{c}k+j \\ k\end{array}\right) \cdot\left(\begin{array}{c}k \\ j\end{array}\right)$.

Finally, we have all the pieces to prove Lemma 5.6 restated below.

Lemma 5.33 (Swap Quadratic Form (restatement of Lemma 5.6)). Let $f=\sum_{i=0}^{k} f_{i}$ with $f_{i} \in C_{i}^{k}$. Suppose $X(\leq d)$ is a $\gamma$-HDX with $d \geq 2 k$. If $\gamma \leq \varepsilon\left(64 k^{k+4} 2^{3 k+1}\right)^{-1}$, then

$$
\left\langle\mathrm{S}_{k, k} f, f\right\rangle=\sum_{i=0}^{k} \lambda_{k}(i) \cdot\left\langle f_{i}, f_{i}\right\rangle \pm \varepsilon,
$$

where $\lambda_{k}(i)$ depends on only on $k$ an $i$, i.e., $\lambda_{k}(i)$ is an approximate eigenvalue of $S_{k, k}$ associated to space $C_{i}^{k}$.

Proof. First note that Lemma 5.28 establishes the existence of approximate eigenvalues $\lambda_{k, j}(i)$ of $\mathrm{N}_{k, k}^{(j)}$ corresponding to space $C_{i}^{k}$ for $i=0, \ldots, k$ such that $\lambda_{k, j}(i)$ depends only on $k, i$ and $j$. To apply Lemma 5.8 we need to bound $\sum_{j=0}^{k}\left|\alpha_{j}\right|$. Since

$$
\sum_{j=0}^{k}\left|\alpha_{j}\right|=\sum_{j=0}^{k}\left(\begin{array}{c}
k+j \\
k
\end{array}\right) \cdot\left(\begin{array}{l}
k \\
j
\end{array}\right) \leq 2^{k} \cdot \sum_{j=0}^{k}\left(\begin{array}{c}
k+j \\
k
\end{array}\right) \leq 2^{3 k+1}
$$

we are done.

\subsection{Rectangular Swap Walks $\mathrm{S}_{k, l}$}

We turn to the spectral analysis of rectangular swap walks, i.e., $\mathrm{S}_{k, l}$ where $k \neq l$. Recall that to bound $\sigma_{2}\left(S_{k, k}\right)$ in Section 5.1 we proved that the spectrum of $S_{k, k}$ for a $\gamma$-HDX is close to the spectrum of $S_{k, k}^{\Delta}$ using the analysis of quadratic forms over balanced operators from Section 5.3. Then we appealed to the fact that $S_{k, k}^{\Delta}$ is expanding since it is the walk operator of the well known Kneser graph. In this rectangular case, we do not have a classical result establishing that $S_{k, l}^{\Delta}$ is expanding, but we were able to establish it Lemma 5.34.

Lemma 5.34. Let $d \geq k+l$ and $\Delta_{d}(n)$ be the complete complex. The second largest singular value $\sigma_{2}\left(\mathrm{~S}_{k, l}^{\Delta}\right)$ of the swap operator $\mathrm{S}_{k, l}^{\Delta}$ on $\Delta_{d}(n)$ is

$$
\sigma_{2}\left(\mathrm{~S}_{k, l}^{\Delta}\right) \leq \max \left(\frac{k}{n-k}, \frac{l}{n-l}\right)
$$

provided $n \geq M_{k, l}$ where $M_{k, l} \in \mathbb{N}$ only depends on $k$ and $l$.

Towards proving Lemma 5.34 we first introduce a generalization of Kneser graphs which we denote bipartite Kneser graphs defined as follows. 
Definition 5.35 (General Bipartite Kneser Graph). Let $X(\leq d)$ where $d \geq k+l$. We denote by $K^{X}(n, k, l)$ the bipartite graph on (vertex) partition $(X(k), X(l))$ where $\mathfrak{s} \in X(k)$ is adjacent to $\mathfrak{t} \in X(l)$ if and only if $\mathfrak{s} \cap \mathfrak{t}$ is empty. We also refer to graphs of the form $K^{X}(n, k, l)$ as bipartite Kneser graphs.

It will be convenient to distinguish bipartite Kneser graphs coming from general $\gamma$-HDX and the complete complex $\Delta_{d}(n)$.

Definition 5.36 (Complete Bipartite Kneser Graph). Let $X(\leq d)$ where $d \geq k+l$. If $X$ is the complete complex, i.e., $X=\Delta_{d}(n)$, then we denote $K^{X}(n, k, l)$ as simply as $K(n, k, l)$ and we refer to it as complete bipartite Kneser.

We obtain the spectra of bipartite Kneser graphs generalizing ${ }^{6}$ the classical result of Fact 5.4. More precisely, we prove Lemma 5.37.

Lemma 5.37 (Bipartite Kneser Spectrum). The non-zero eigenvalues of the (normalized) walk operator of $K(n, k, l)$ are $\pm \lambda_{i}$ where

$$
\lambda_{i}=\frac{\left(\begin{array}{c}
n-k-i \\
l-i
\end{array}\right)\left(\begin{array}{c}
n-l-i \\
k-i
\end{array}\right)}{\left(\begin{array}{c}
n-k \\
l
\end{array}\right)\left(\begin{array}{c}
n-l \\
k
\end{array}\right)}
$$

for $i=0, \ldots, \min (k, l)$.

Now the proof follows a similar strategy to the $S_{k, k}$, namely, we analyze quadratic forms over $S_{k, k}$ using the results from Section 5.3

Let $X(\leq d)$ where $d \geq k+l$. Let $A_{k, l}$ be the (normalized) walk operator of $K^{X}(n, k, l)$, i.e.,

$$
\mathrm{A}_{k, l}=\left(\begin{array}{cc}
0 & \mathrm{~S}_{k, l}^{(l)} \\
\left(\mathrm{S}_{k, l}^{(l)}\right)^{\dagger} & 0
\end{array}\right)
$$

To determine the spectrum of $\mathrm{A}_{k, l}$ it is enough to consider the spectrum of $\mathrm{B}=\mathrm{S}_{k, l}^{(l)}\left(\mathrm{S}_{k, l}^{(l)}\right)^{\dagger}$. Using Corollary 4.13, we have

$$
\begin{aligned}
\mathrm{B}= & \left(\sum_{j=0}^{l}(-1)^{l-j}\left(\begin{array}{c}
k+j \\
l
\end{array}\right) \cdot\left(\begin{array}{l}
l \\
j
\end{array}\right) \cdot \mathrm{N}_{k, l}^{(j)}\right) \\
& \left(\sum_{j^{\prime}=0}^{l}(-1)^{l-j^{\prime}}\left(\begin{array}{c}
k+j^{\prime} \\
l
\end{array}\right) \cdot\left(\begin{array}{c}
l \\
j^{\prime}
\end{array}\right) \cdot\left(\mathrm{N}_{k, l}^{\left(j^{\prime}\right)}\right)^{+}\right)=\sum_{j, j^{\prime}=0}^{l} \alpha_{k, l, j, j^{\prime}} \mathrm{N}_{k, l}^{(j)} \mathrm{N}_{l, k}^{\left(j^{\prime}+k-l\right)},
\end{aligned}
$$

for some coefficients $\alpha_{k, l, j, j^{\prime}}$ depending only on $k, l, i, j$ and $j^{\prime}$. Since we have not yet used any specific property of HDXs, these coefficients are the same for the complete complex and general HDXs.

Lemma 5.38. Let $X(\leq d)$ be a $\gamma$-HDX with $d \geq k+$ l. Let $f=\sum_{i=0}^{k} f_{i}$ with $f_{i} \in C_{i}^{k}$. For $\varepsilon \in(0,1)$, if $\gamma \leq \varepsilon\left(64 k^{k+2} \ell^{2} 2^{2 k+4 l+2}\right)^{-1}$, then

$$
\langle\mathrm{B} f, f\rangle=\sum_{i=0}^{k}\left(\sum_{j, j^{\prime}=0}^{l} \alpha_{k, l, j, j^{\prime}} \lambda_{k, l, j, j^{\prime}}(i)\right) \cdot\left\langle f_{i}, f_{i}\right\rangle+\varepsilon,
$$

\footnotetext{
${ }^{6}$ Note that the singular values of $K(n, k)$ can be deduced from the bipartite case.
} 
where $\lambda_{k, l, j, j^{\prime}}(i)$ is the approximate eigenvalues of $\mathrm{N}_{k, l}^{(j)} \mathrm{N}_{l, k}^{\left(j^{\prime}+k-l\right)}$ corresponding to space $C_{i}^{k}$. Furthermore, $\lambda_{k, l, j, j j^{\prime}}(i)$ depends only on $k, l, i, j$ and $j^{\prime}$.

Proof. First observe that each $\mathrm{N}_{k, l}^{(j)} \mathrm{N}_{l, k}^{\left(j^{\prime}+k-l\right)}$ maps $C^{k}$ to itself, so it is a product of the same number of up and down operators. Now to apply Lemma 5.8 it only remains to bound $\sum_{j, j^{\prime}=0}^{l}\left|\alpha_{k, l, j, j^{\prime}}\right|$. Since

$$
\begin{aligned}
\sum_{j, j^{\prime}=0}^{l}\left|\alpha_{k, l, j, j^{\prime}}\right| & =\sum_{j, j^{\prime}=0}^{l}\left(\begin{array}{c}
k+j \\
l
\end{array}\right) \cdot\left(\begin{array}{l}
l \\
j
\end{array}\right)\left(\begin{array}{c}
k+j^{\prime} \\
l
\end{array}\right) \cdot\left(\begin{array}{l}
l \\
j^{\prime}
\end{array}\right) \\
& \leq 2^{2 l}\left(\sum_{j=0}^{l}\left(\begin{array}{c}
k+j \\
l
\end{array}\right)\right) \cdot\left(\sum_{j^{\prime}=0}^{l}\left(\begin{array}{c}
k+j^{\prime} \\
l
\end{array}\right)\right) \leq 2^{2 k+4 l+2},
\end{aligned}
$$

we are done.

Let $B$ and $B^{\Delta}$ stand for the $B$ operator for general $\gamma$-HDX and the complete complex, respectively.

Lemma 5.39. Suppose $X(\leq d)$ is a $\gamma-H D X$ with $d \geq k+l$. For $\varepsilon \in(0,1)$, if $\gamma \leq \varepsilon^{2}\left(64 k^{k+2} \ell^{2} 2^{2 k+4 l+2}\right)^{-1}$, then the second largest singular value $\sigma_{2}(\mathrm{~B})$ of $\mathrm{B}$ is

$$
\sigma_{2}(\mathrm{~B}) \leq \varepsilon^{2} .
$$

Furthermore, the second largest non-trivial eigenvalue $\lambda\left(\mathrm{A}_{k, l}\right)$ of the walk matrix of $K(n, k, l)$ is

$$
\lambda\left(\mathrm{A}_{k, l}\right) \leq \varepsilon
$$

Proof. The proof follows the same strategy of Theorem 5.1, namely, we first consider $\mathrm{B}^{\Delta}$ and show that $\sum_{j, j^{\prime}=0}^{l} \alpha_{k, l, j, j^{\prime}} \lambda_{k, l, j, j^{\prime}}(i)=0$. Using Lemma 5.34, we deduce that

$$
\left|\sum_{j, j^{\prime}=0}^{l} \alpha_{k, l, j, j^{\prime}} \lambda_{k, l, j, j j^{\prime}}(i)\right|=O_{k, l}\left(\frac{1}{n^{2}}\right)
$$

for $i \in[k]$ where in this range each $C_{i}^{k}$ is not the trivial approximate eigenspace (associated with eigenvalue 1). Since $\alpha_{k, l, j, j^{\prime}}$ and $\lambda_{k, l, j, j^{\prime}}(i)$ do not depend on $n$ and $n$ is arbitrary, the LHS above is actually zero. Then our choice of $\gamma$ Lemma 5.8 gives

$$
\max _{f \in C^{k}: f \perp 1,\|f\|=1}|\langle\mathrm{~B} f, f\rangle| \leq \max _{i \in[k]}\left|\sum_{j, j^{\prime}=0}^{l} \alpha_{k, l, j, j j^{\prime}} \lambda_{k, l, j, j^{\prime}}(i)\right|+\varepsilon^{2}=\varepsilon^{2} .
$$

Now the proof of Theorem 5.2 follows. For convenience, we restate it.

Theorem 5.40 (Rectangular Swap Walk Spectral Bound (restatement of Theorem 5.2)). Suppose $X(\leq d)$ is a $\gamma$-HDX with $d \geq k+l$ and $k \leq l$. For $\sigma \in(0,1)$, if $\gamma \leq \sigma^{2} \cdot\left(64 k^{k+2} \ell^{2} 2^{2 k+4 l+2}\right)^{-1}$, then the largest non-trivial singular value $\sigma_{2}\left(S_{k, l}\right)$ of the swap operator $S_{k, l}$ is

$$
\sigma_{2}\left(\mathrm{~S}_{k, l}\right) \leq \sigma .
$$

Proof. Follows directly from Lemma 5.39. 


\subsection{Bipartite Kneser Graphs - Complete Complex}

Now we determine the spectrum of the complete bipartite Kneser graph $K(n, k, l)$. More precisely, we prove the following.

Lemma 5.41 (Bipartite Kneser Spectrum (restatement of Lemma 5.37)). The non-zero eigenvalues of the normalized walk operator of $K(n, k, l)$ are $\pm \lambda_{i}$ where

$$
\lambda_{i}=\frac{\left(\begin{array}{c}
n-k-i \\
l-i
\end{array}\right)\left(\begin{array}{c}
n-l-i \\
k-i
\end{array}\right)}{\left(\begin{array}{c}
n-k \\
l
\end{array}\right)\left(\begin{array}{c}
n-l \\
k
\end{array}\right)},
$$

for $i=0, \ldots, \min (k, l)$.

Henceforth, set $X=\Delta_{d}(n)$. To prove Lemma 5.37 we work with the natural rectangular matrix associated with $K(n, k, l)$, namely, the matrix $W \in \mathbb{R}^{X(k) \times X(l)}$ such that

$$
W(\mathfrak{s}, \mathfrak{t})=\mathbb{1}_{[\mathfrak{s} \cap \mathfrak{t}=\varnothing]}
$$

for every $\mathfrak{s} \in X(k)$ and $\mathfrak{t} \in X(l)$.

Observe that the entries of $W W^{\top}$ and $W^{\top} W$ only depend on the size of the intersection of the sets indexing the row and columns. Hence, these matrices belong to the Johnson scheme [GM15] $J(n, k)$ and $J(n, l)$, respectively. Moreover, the left and right singular vectors of $W$ are eigenvectors of these schemes.

We adopt the eigenvectors used in Filmus' work [Fil16], i.e., natural basis vectors coming from some irreducible representation of $S_{n}$ (see [Sag13]). First we introduce some notation. Let $\mu=(n-i, i)$ be a partition of $n$ and let $\tau_{\mu}$ be a standard tableau of shape $\mu$. Suppose the first row $\tau_{\mu}$ contains $a_{1}<\cdots<a_{n-i}$ whereas the second contains $b_{1}<\cdots<b_{i}$. To $\tau_{\mu}$ we associate the function $\varphi_{\tau_{\mu}} \in \mathbb{R}^{\left(\begin{array}{ll}(n) \\ k\end{array}\right)}$ as follows

$$
\varphi_{\tau_{\mu}}=\left(\mathbb{1}_{a_{1}}-\mathbb{1}_{b_{1}}\right) \ldots\left(\mathbb{1}_{a_{i}}-\mathbb{1}_{b_{i}}\right),
$$

where $\mathbb{1}_{a} \in \mathbb{R}_{k}^{\left(\begin{array}{l}n \\ k\end{array}\right)}$ is the containment indicator of element $a$, i.e., $\mathbb{1}_{a}(\mathfrak{s})=1$ if and only if $a \in \mathfrak{s}$. Filmus proved that

$$
\left\{\varphi_{\tau_{\mu}} \mid 0 \leq i \leq k, \mu \vdash(n-i, i), \tau_{\mu} \text { standard }\right\}
$$

is an eigenbasis of $\mathcal{J}(n, k)$. We abuse the notation by considering $\varphi_{\tau_{\mu}}$ as both a function in

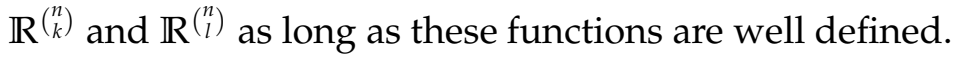

Claim 5.42. If $\mu=(n-i, i)$ and $k, l \geq i$, then

$$
\mathrm{W} \varphi_{\tau_{\mu}}=(-1)^{i} \cdot\left(\begin{array}{c}
n-k-i \\
l-i
\end{array}\right) \cdot \varphi_{\tau_{\mu}} .
$$

Proof. We follow a similar strategy of Filmus. For convenience suppose $\varphi_{\tau_{\mu}}=\left(\mathbb{1}_{1}-\right.$ $\left.\mathbb{1}_{2}\right) \ldots\left(\mathbb{1}_{2 i-1}-\mathbb{1}_{2 i}\right)$. For $i=0$ the claim follows immediately so assume $i \geq 1$. Consider $\left(\mathrm{W} \varphi_{\tau_{\mu}}\right)(\mathfrak{s})$ where $\mathfrak{s} \in\left(\begin{array}{c}{[n]} \\ k\end{array}\right)$. Note that

$$
\left(\mathrm{W} \varphi_{\tau_{\mu}}\right)(\mathfrak{s})=\sum_{\mathfrak{t} \in Y: \mathfrak{s} \cap \mathfrak{t}=\varnothing} \varphi_{\tau_{\mu}}(\mathfrak{t}) .
$$


If $2 j-1,2 j \in \mathfrak{s}$ for some $j \in[i]$, then $2 j-1,2 j \notin \mathfrak{t}$ so $\varphi_{\tau_{\mu}}(\mathfrak{s})=0=\left(W \varphi_{\tau_{\mu}}\right)(\mathfrak{s})$. If $2 j-1,2 j \notin \mathfrak{s}$ for some $j \in[i]$, for each $\mathfrak{t}$ adjacent to $\mathfrak{s}$ there four cases: $2 j-1,2 j \in \mathfrak{t}$, $2 j-1,2 j \notin \mathfrak{t}, 2 j-1 \in \mathfrak{t}$ and $2 j \notin \mathfrak{t}$ or vice-versa. The first two cases yield $\varphi_{\tau_{\mu}}(\mathfrak{t})=0$ while the last two cases cancel each other in the summation and again $\varphi_{\tau_{\mu}}(\mathfrak{s})=0=\left(W \varphi_{\tau_{\mu}}\right)(\mathfrak{s})$. Now suppose that $\mathfrak{s}$ contains exactly one element of each pair $2 j-1,2 j$. For any adjacent $\mathfrak{t}$ to yield $\varphi_{\tau_{\mu}}(\mathfrak{t}) \neq 0, \mathfrak{t}$ must contain $[2 i] \backslash \mathfrak{s}$. Since there are $\left(\begin{array}{c}n-k-i \\ l-i\end{array}\right)$ such possibilities for $\mathfrak{t}$ we obtain

$$
\mathrm{W} \varphi_{\tau_{\mu}}=(-1)^{i} \cdot\left(\begin{array}{c}
n-k-i \\
l-i
\end{array}\right) \cdot \varphi_{\tau_{\mu}}
$$

where the sign $(-1)^{i}$ follows from the product of the signs of each the $i$ pairs and the fact that $\mathfrak{s}$ and $t$ partition the elements in each pair.

Since we are working with singular vectors, we need to be careful with their normalization when deriving the singular values. We stress that the norm of $\varphi_{\tau_{\mu}}$ depends on the space where $\varphi_{\tau_{\mu}}$ lies.

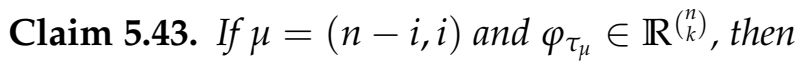

$$
\left\|\varphi_{\tau_{\mu}}\right\|_{2}=\sqrt{2^{i}\left(\begin{array}{c}
n-2 i \\
k-i
\end{array}\right)} .
$$

Proof. Since $\varphi_{\tau_{\mu}}$ assumes values in $\{-1,0,1\}$ so its enough to count the number of sets $\mathfrak{s} \in\left(\begin{array}{c}{[n]} \\ k\end{array}\right)$ such that $\varphi_{\tau_{\mu}}(\mathfrak{s}) \neq 0$. To have $\varphi_{\tau_{\mu}}(\mathfrak{s}) \neq 0, \mathfrak{s}$ must contain exactly one element in each pair and the remaining $k-i$ elements of $\mathfrak{s}$ can be chosen arbitrarily among the elements avoiding the $2 i$ elements appearing in the indicators defining $\varphi_{\tau_{\mu}}$.

Now the singular values of $W$ follow.

Corollary 5.44 (Singular Values). The singular values of $W$ are

$$
\sigma_{i}=\left(\begin{array}{c}
n-k-i \\
l-i
\end{array}\right) \cdot \frac{\left\|\varphi_{\tau_{\mu}}^{k}\right\|_{2}}{\left\|\varphi_{\tau_{\mu}}^{l}\right\|_{2}}
$$

for $i=0, \ldots, \min (k, l)$.

Note that for $k=l$ we recover the well know result of Fact 5.4.

Finally we compute the eigenvalues of the bipartite graph $K(n, k, l)$. Let $\mathrm{A}_{n, k, l}$ be its normalized adjacency matrix, i.e.,

$$
\mathrm{A}_{n, k, l}=\left(\begin{array}{cc}
0 & \frac{1}{\left(\begin{array}{c}
n-k \\
l
\end{array}\right)} \mathrm{W} \\
\frac{1}{\left(\begin{array}{c}
n-l \\
k
\end{array}\right)} \mathrm{W}^{\top} & 0
\end{array}\right) .
$$

Lemma 5.45 (Bipartite Kneser Spectrum (restatement of Lemma 5.37)). The non-zero eigenvalues of the normalized walk operator of $K(n, k, l)$ are $\pm \lambda_{i}$ where

$$
\lambda_{i}=\frac{\left(\begin{array}{c}
n-k-i \\
l-i
\end{array}\right)\left(\begin{array}{c}
n-l-i \\
k-i
\end{array}\right)}{\left(\begin{array}{c}
n-k \\
l
\end{array}\right)\left(\begin{array}{c}
n-l \\
k
\end{array}\right)}
$$

for $i=0, \ldots, \min (k, l)$. 
Proof. Since the spectrum of a bipartite graph is symmetric around zero, it is enough to compute the eigenvalues of $A_{n, k, l}^{2}$. Set $\alpha=1 /\left(\begin{array}{c}n-k \\ l\end{array}\right)\left(\begin{array}{c}n-l \\ k\end{array}\right)$. Moreover, we consider $\alpha \cdot \mathrm{WW}^{\top}$ since $\alpha \cdot W^{\top} W$ has the same non-zero eigenvalues. The non-zero eigenvalues of $\alpha \cdot W^{\top}$ are

$$
\lambda_{i}=\frac{\left(\begin{array}{c}
n-k-i \\
l-i
\end{array}\right)\left(\begin{array}{c}
n-l-i \\
k-i
\end{array}\right)}{\left(\begin{array}{c}
n-k \\
l
\end{array}\right)\left(\begin{array}{c}
n-l \\
k
\end{array}\right)},
$$

for $i=0, \ldots, \min (k, l)$.

\section{Approximating Max- $k-\mathrm{CSP}$}

In the following, we will show that $k$-CSP instances $\mathfrak{I}$ whose constraint complex $X_{\mathfrak{I}}(\leq$ $k$ ) is a suitable expander admit an efficient approximation algorithm. We will assume throughout that $X_{\mathfrak{I}}(1)=[n]$, and drop the subscript $\mathfrak{I}$.

This was shown for 2-CSPs in [BRS11]. In extending this result to $k$-CSPs we will rely on a central Lemma of their paper. Before, we explain our algorithm we give a basic outline of our idea:

We will work with the SDP relaxation for the $k$-CSP problem given by L-levels of SoS hierarchy, as defined in Section 2.4 (for $L$ to be specified later). This will give us an $L$ local PSD ensemble $\left\{\mathbf{Y}_{1}, \ldots, \mathbf{Y}_{n}\right\}$, which attains some value $\operatorname{SDP}(\mathfrak{I}) \geq \operatorname{OPT}(\mathfrak{I})$. Since $\left\{\mathbf{Y}_{1}, \ldots, \mathbf{Y}_{n}\right\}$, is a local PSD ensemble, and not necessarily a probability distribution, we cannot sample from it directly. Nevertheless, since $\left\{\mathbf{Y}_{j}\right\}$ will be actual probability distributions for all $j \in[n]$, one can independently sample $\eta_{j} \sim\left\{\mathbf{Y}_{j}\right\}$ and use $\eta=\left(\eta_{1}, \ldots, \eta_{n}\right)$ as the assignment for the $k$-CSP instance $\mathfrak{I}$.

Unfortunately, while we know that the local distributions $\left\{\mathbf{Y}_{\mathfrak{a}}\right\}_{\mathfrak{a} \in X(k)}$ induced by $\left\{\mathbf{Y}_{1}, \ldots, \mathbf{Y}_{n}\right\}$ will satisfy the constraints of $\mathfrak{I}$ with good probability, i.e.,

$$
\underset{\mathfrak{a} \sim \Pi_{k}}{\mathbb{E}} \underset{\left\{\mathbf{Y}_{\mathfrak{a}}\right\}}{\mathbb{E}}\left[\mathbf{1}[\underbrace{\mathbf{Y}_{a} \text { satisfies the constraint on } \mathfrak{a}}_{\Longleftrightarrow \mathbf{Y}_{\mathfrak{a}} \in \mathcal{C}_{\mathfrak{a}}}]=\operatorname{SDP}(\mathfrak{I}) \geq \operatorname{OPT}(\mathfrak{I}),\right.
$$

this might not be the case for the assignment $\eta$ sampled as before. It might be that the random variables $\mathbf{Y}_{a_{1}}, \ldots, \mathbf{Y}_{a_{k}}$ are highly correlated for $\mathfrak{a} \in X(k)$, i.e.,

$$
\underset{\mathfrak{a} \sim \Pi_{k}}{\mathbb{E}}\left\|\left\{\mathbf{Y}_{\mathfrak{a}}\right\}-\left\{\mathbf{Y}_{a_{1}}\right\} \cdots\left\{\mathbf{Y}_{a_{k}}\right\}\right\|_{1}
$$

is large. One strategy employed by [BRS11] to ensure that the quantity above is small, is making the local PSD ensemble $\left\{\mathbf{Y}_{1}, \ldots, \mathbf{Y}_{n}\right\}$ be consistent with a randomly sampled partial assignment for a small subset of variables (q.v. Section 2.4). We will show that this strategy is succesful if $X(\leq k)$ is a $\gamma$-HDX (for $\gamma$ sufficiently small). Our final algorithm will be the following, 


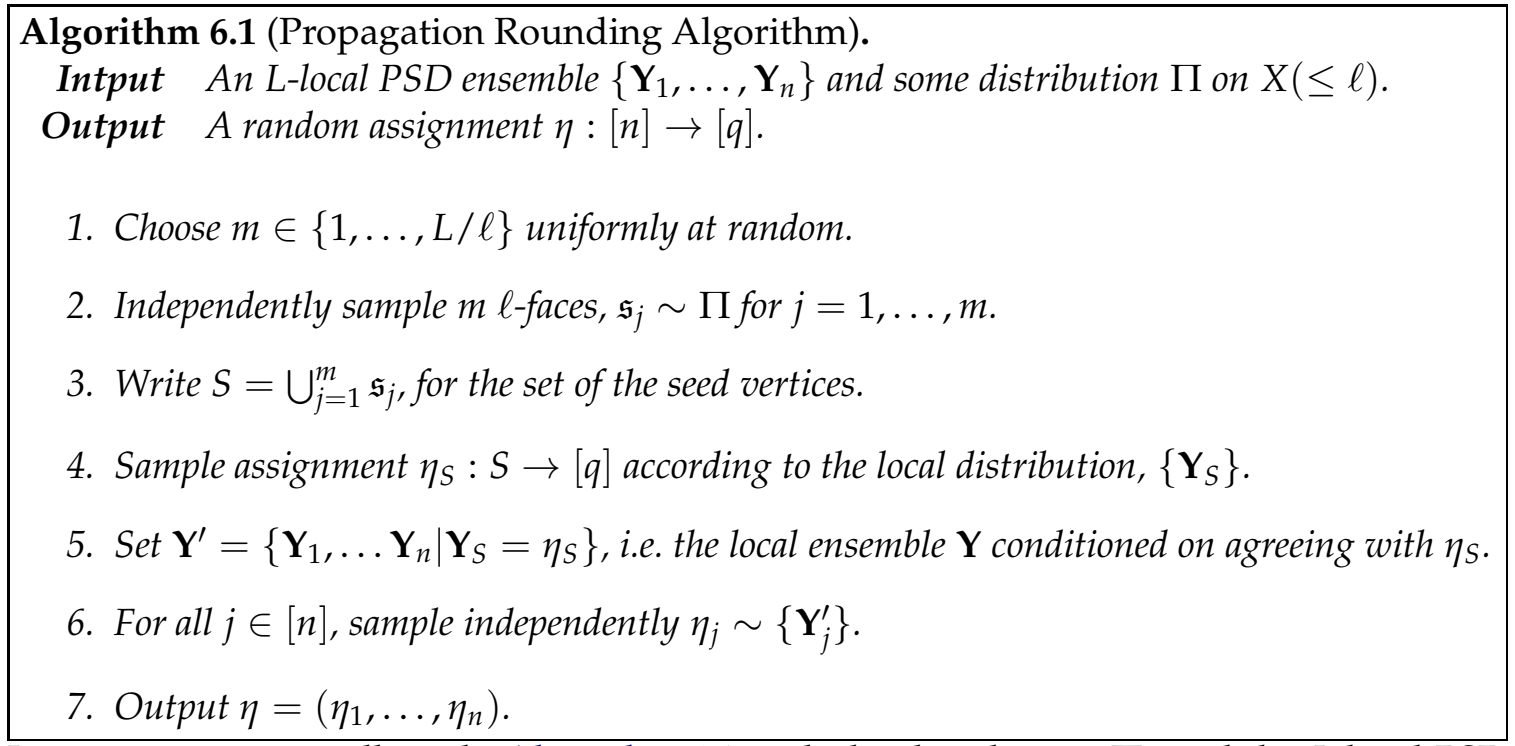

In our setting, we will apply Algorithm 6.1 with the distribution $\Pi_{k}$ and the L-local PSD ensemble $\left\{\mathbf{Y}_{1}, \ldots, \mathbf{Y}_{n}\right\}$. Notice that in expectation, the marginals of $\mathbf{Y}^{\prime}$ on faces $\mathfrak{a} \in X(k)$ - which are actual distributions - will agree with the marginals of $\mathbf{Y}$, i.e. $\mathbb{E}_{S, \eta_{S}} \mathbb{E} \mathbf{Y}_{\mathfrak{a}}^{\prime}=$ $\mathbb{E} \mathbf{Y}_{\mathfrak{a}}$. In particular, the approximation quality of Algorithm 6.1 will depend on the average correlation of $\mathbf{Y}_{a_{1}}^{\prime}, \ldots, \mathbf{Y}_{a_{k}}^{\prime}$ on the constraints $\mathfrak{a} \in X(k)$, where $\mathbf{Y}^{\prime}$ is the local PSD ensemble obtained at the end of the first phase of Algorithm 6.1.

In the case where $k=2$, the following is known

Theorem 6.2 (Theorem 5.6 from [BRS11]). Suppose a weighted undirected graph $G=\left([n], E, \Pi_{2}\right)$ and an L-local PSD ensemble $Y=\left\{\mathbf{Y}_{1}, \ldots, \mathbf{Y}_{n}\right\}$ are given. There exists absolute constants $c \geq 0$ and $C \geq 0$ satisfying the following: If $L \geq c \cdot \frac{q}{\varepsilon^{4}}, \operatorname{Supp}\left(\mathbf{Y}_{i}\right) \leq q$ for all $i \in V$, and $\lambda_{2}(G) \leq C \cdot \varepsilon^{2} / q^{2}$ then we have

$$
\underset{\{i, j\} \sim \Pi_{2}}{\mathbb{E}}\left\|\left\{\mathbf{Y}_{i}^{\prime}, \mathbf{Y}_{j}^{\prime}\right\}-\left\{\mathbf{Y}_{i}^{\prime}\right\}\left\{\mathbf{Y}_{j}^{\prime}\right\}\right\|_{1} \leq \varepsilon
$$

where $\mathbf{Y}^{\prime}$ is as defined in Algorithm 6.1 on the input of $\left\{\mathbf{Y}_{1}, \ldots, \mathbf{Y}_{n}\right\}$ and $\Pi_{1}$.

To approximate $k$-CSPs well, we will show the following generalization of Theorem 6.2 for $k$-CSP instances $\mathfrak{I}$, whose constraint complex $X(\leq k)$ is $\gamma$-HDX, for $\gamma$ sufficiently small.

Theorem 6.3. Suppose a simplicial complex $X(\leq k)$ with $X(1)=[n]$ and an L-local PSD ensemble $\mathbf{Y}=\left\{\mathbf{Y}_{1}, \ldots, \mathbf{Y}_{n}\right\}$ are given.

There exists some universal constants $c^{\prime} \geq 0$ and $C^{\prime} \geq 0$ satisfying the following: If $L \geq$ $c^{\prime} \cdot\left(q^{k} \cdot k^{5} / \varepsilon^{4}\right), \operatorname{Supp}\left(\mathbf{Y}_{j}\right) \leq q$ for all $j \in[n]$, and $X$ is a $\gamma$-HDX for $\gamma \leq C^{\prime} \cdot \varepsilon^{4} /\left(k^{8+k} \cdot 2^{6 k} \cdot q^{2 k}\right)$. Then, we have

$$
\underset{\mathfrak{a} \sim \Pi_{k}}{\mathbb{E}}\left\|\left\{\mathbf{Y}_{\mathfrak{a}}^{\prime}\right\}-\left\{\mathbf{Y}_{a_{1}}^{\prime}\right\} \cdots\left\{\mathbf{Y}_{a_{k}}^{\prime}\right\}\right\|_{1} \leq \varepsilon,
$$

where $\mathbf{Y}^{\prime}$ is as defined in Algorithm 6.1 on the input of $\left\{\mathbf{Y}_{1}, \ldots, \mathbf{Y}_{n}\right\}$ and $\Pi_{k}$.

Indeed, using Theorem 6.3, it will be straightforward to prove the following,

Corollary 6.4. Suppose $\mathfrak{I}$ is a $q$-ary $k$-CSP instance whose constraint complex $X(\leq k)$ is a $\gamma$ HDX. 
There exists absolute constants $C^{\prime} \geq 0$ and $c^{\prime} \geq 0$, satisfying the following: If $\gamma \leq C^{\prime}$. $\varepsilon^{4} /\left(k^{8+k} \cdot 2^{6 k} \cdot q^{2 k}\right)$, there is an algorithm that runs in time $n^{O}\left(k^{5} \cdot q^{2 k} \cdot \varepsilon^{-4}\right)$ based on $\left(\frac{c^{\prime} \cdot k^{5} \cdot q^{k}}{\varepsilon^{4}}\right)$-levels of SoS-hierarchy and Algorithm 6.1 that outputs a random assignment $\eta:[n] \rightarrow[q]$ that in expectation ensures $\operatorname{SAT}_{\mathfrak{I}}(\eta)=\mathrm{OPT}(\mathfrak{I})-\varepsilon$.

Proof of Corollary 6.4. The algorithm will just run Algorithm 6.1 on the local PSD-ensemble $\left\{\mathbf{Y}_{1}, \ldots, \mathbf{Y}_{n}\right\}$ given by the SDP relaxation of $\mathfrak{I}$ strengthened by $L=c^{\prime} \cdot \frac{k^{5} \cdot q^{2 k}}{\varepsilon^{4}}$-levels of SoShierarchy and $\Pi_{k}$ - where $c^{\prime} \geq 0$ is the constant from Theorem 6.3. Y satisfies,

$$
\operatorname{SDP}(\mathfrak{I})=\underset{\mathfrak{a} \sim \Pi_{k}}{\mathbb{E}}\left[\underset{\left\{\mathbf{Y}_{\mathfrak{a}}\right\}}{\mathbb{E}}\left[\mathbf{1}\left[\mathbf{Y}_{\mathfrak{a}} \in \mathcal{C}_{\mathfrak{a}}\right]\right]\right] \geq \operatorname{OPT}(\mathfrak{I})
$$

Let $S, \eta_{S}$, and $\mathbf{Y}^{\prime}$ be defined as in Algorithm 6.1 on the input of $\mathbf{Y}$ and $\Pi_{k}$. Since the conditioning done on $\left\{\mathbf{Y}^{\prime}\right\}$ is consistent with the local distribution, by law of total expectation and Eq. (6) one has

$$
\underset{S}{\mathbb{E}} \underset{\eta_{\mathcal{S}} \sim\left\{\mathbf{Y}_{S}\right\}}{\mathbb{E}} \underset{\mathfrak{a} \sim \Pi_{k}}{\mathbb{E}} \underset{\left\{\mathbf{Y}_{\mathfrak{a}}^{\prime}\right\}}{\mathbb{E}}\left[\mathbf{1}\left[\mathbf{Y}_{\mathfrak{a}}^{\prime} \in \mathcal{C}_{\mathfrak{a}}\right]\right]=\operatorname{SDP}(\mathfrak{I}) \geq \operatorname{OPT}(\mathfrak{I})
$$

By Theorem 6.3 we know that

$$
\underset{S}{\mathbb{E}} \underset{\eta_{S} \sim\left\{\mathbf{Y}_{S}\right\}}{\mathbb{E}} \underset{\mathfrak{a} \sim \Pi_{k}}{\mathbb{E}}\left\|\left\{\mathbf{Y}_{\mathfrak{a}}^{\prime}\right\}-\left\{\mathbf{Y}_{a_{1}}^{\prime}\right\} \cdots\left\{\mathbf{Y}_{a_{k}}^{\prime}\right\}\right\|_{1} \leq \varepsilon
$$

Now, the fraction of constraints satisfied by the algorithm in expectation is

$$
\underset{\eta}{\mathbb{E}}\left[\operatorname{SAT}_{\mathfrak{I}}(\eta)\right]=\underset{S}{\mathbb{S}} \underset{\eta_{\mathcal{S}} \sim\left\{\mathbf{Y}_{S}\right\}}{\mathbb{E}} \underset{\mathfrak{a} \sim \Pi_{k}}{\mathbb{E}} \underset{\left(\eta_{1}, \ldots, \eta_{n}\right) \sim\left\{\mathbf{Y}_{1}^{\prime}\right\} \cdots\left\{\mathbf{Y}_{n}^{\prime}\right\}}{\mathbb{E}}\left[\mathbf{1}\left[\left.\eta\right|_{\mathfrak{a}} \in \mathcal{C}_{\mathfrak{a}}\right]\right] .
$$

By using Eq. (8), we can obtain

$$
\underset{\eta}{\mathbb{E}}\left[\operatorname{SAT}_{\mathfrak{I}}(\eta)\right] \geq \underset{S}{\mathbb{E}}\left[\underset{\eta_{S} \sim\left\{\mathbf{Y}_{S}\right\}}{\mathbb{E}} \underset{\left\{\mathbf{Y}_{\mathfrak{a}}\right\}}{\mathbb{E}} \mathbf{1}\left[\mathbf{Y}_{\mathfrak{a}}^{\prime} \text { satisfies the constraint on } \mathfrak{a}\right]\right]-\varepsilon .
$$

Using Eq. (7), we can conclude

$$
\underset{\eta}{\mathbb{E}}\left[\operatorname{SAT}_{\mathfrak{I}}(\eta)\right] \geq \operatorname{SDP}(\mathfrak{I})-\varepsilon=\operatorname{OPT}(\mathfrak{I})-\varepsilon .
$$

Our proof of Theorem 6.3 will hinge on the fact that we can upper-bound the expected correlation of a face of large cardinality $\ell$, in terms of expected correlation over faces of smaller cardinality and expected correlations along the edges of a swap graph. The swap graph here is defined as a weighted graph $G_{\ell_{1}, \ell_{2}}=\left(X\left(\ell_{1}\right) \sqcup X\left(\ell_{2}\right), E\left(\ell_{1}, \ell_{2}\right), w_{\ell_{1}, \ell_{2}}\right)$, where

$$
E\left(\ell_{1}, \ell_{2}\right)=\left\{\{\mathfrak{a}, \mathfrak{b}\}: \mathfrak{a} \in X\left(\ell_{1}\right), \mathfrak{b} \in X\left(\ell_{2}\right) \text {, and } \mathfrak{a} \sqcup \mathfrak{b} \in X\left(\ell_{1}+\ell_{2}\right)\right\} .
$$

We will assume $\ell_{1} \geq \ell_{2}$, and if $\ell_{1}=\ell_{2}$ we are going to identify the two copies of every vertex. We will endow $E\left(\ell_{1}, \ell_{2}\right)$ with the weight function,

$$
w_{\ell_{1}, \ell_{2}}(\mathfrak{a}, \mathfrak{b})=\frac{\Pi_{\ell_{1}+\ell_{2}}(\mathfrak{a} \sqcup \mathfrak{b})}{\left(\begin{array}{c}
\ell_{1}+\ell_{2} \\
\ell_{1}
\end{array}\right)},
$$


which can easily be verified to be a probability distribution on $E\left(\ell_{1}, \ell_{2}\right)$ Notice that in the case where $\ell_{1} \neq \ell_{2}$ the random walk matrix of $G_{\ell_{1}, \ell_{2}}$ is given by

$$
A_{\ell_{1}, \ell_{2}}=\left(\begin{array}{cc}
0 & S_{\ell_{1}, \ell_{2}} \\
S_{\ell_{1}, \ell_{2}}^{+} & 0
\end{array}\right)
$$

and if $\ell_{1}=\ell_{2}$ we have $A_{\ell_{1}, \ell_{1}}=S_{\ell_{1}, \ell_{1}}$. The stationary distribution of $A_{\ell_{1}, \ell_{2}}$ is $\Pi_{\ell_{1}, \ell_{2}}$ defined by,

$$
\Pi_{\ell_{1}, \ell_{2}}(\mathfrak{b})=\mathbf{1}\left[\mathfrak{b} \in X\left(\ell_{1}\right)\right] \cdot \frac{1}{2} \cdot \Pi_{\ell_{1}}(\mathfrak{b})+\mathbf{1}\left[\mathfrak{b} \in X\left(\ell_{2}\right)\right] \cdot \frac{1}{2} \cdot \Pi_{\ell_{2}}(\mathfrak{b}) .
$$

When we write an expectation of $\boldsymbol{f}(\bullet, \bullet)$ over the edges in $E\left(\ell_{1}, \ell_{2}\right)$ with respect to $w_{\ell_{1}, \ell_{2}}$, it is important to note,

$$
\underset{\{\mathfrak{s}, \mathfrak{t}\} \sim w_{\ell_{1}, \ell_{2}}}{\mathbb{E}}[\boldsymbol{f}(\mathfrak{s}, \mathfrak{t})]=\sum_{\{\mathfrak{s}, \mathfrak{t}\} \in E\left(\ell_{\ell_{1}, \ell_{2}}\right)} \frac{1}{\left(\begin{array}{c}
\ell_{1}+\ell_{2} \\
\ell_{1}
\end{array}\right)} \cdot \boldsymbol{f}(\mathfrak{s}, \mathfrak{t}) \cdot \Pi_{\ell_{1}+\ell_{2}}(\mathfrak{s} \sqcup \mathfrak{t})=\frac{1}{\left(\begin{array}{c}
\ell \\
\ell_{1}
\end{array}\right)} \underset{\mathfrak{a} \sim \Pi_{k}}{\mathbb{E}}\left[\sum_{\{\mathfrak{s}, \mathfrak{t}\} \sim \mathfrak{a}} f(\mathfrak{s}, \mathfrak{t})\right],
$$

where sum within the expectation in the RHS runs over the $\left(\begin{array}{c}\ell_{1}+\ell_{2} \\ \ell_{1}\end{array}\right)$ possible ways of splitting $\mathfrak{a}$ into $\mathfrak{s} \sqcup \mathfrak{t}$ such that $\mathfrak{s} \in X\left(\ell_{1}\right)$ and $\mathfrak{t} \in X\left(\ell_{2}\right)$. When we are speaking about the spectral expansion of $G_{\ell_{1}, \ell_{2}}$, we will be speaking with regards to $\lambda_{2}\left(G_{\ell_{1}, \ell_{2}}\right)$ and not with regards to $\sigma_{2}\left(G_{\ell_{1}, \ell_{2}}\right)$.

Remark 6.5. By simple linear algebra, we have

$$
\lambda_{2}\left(G_{\ell_{1}, \ell_{2}}\right):=\lambda_{2}\left(\mathrm{~A}_{\ell_{1}, \ell_{2}}\right) \leq \sigma_{2}\left(\mathrm{~S}_{\ell_{1}, \ell_{2}}\right),
$$

where we employ the notation $\lambda_{2}(\mathrm{M})$ to denote the second largest eigenvalue (signed) of the matrix M.

With this, we will show

Lemma 6.6 (Glorified Triangle Inequality). For a simplicial complex $X(\leq k), \ell_{1} \geq \ell_{2} \geq 0$, $\ell=\ell_{1}+\ell_{2}, \ell \leq k$, and an $\ell$-local ensemble $\left\{\mathbf{Y}_{1}, \ldots, \mathbf{Y}_{n}\right\}$, one has

$$
\begin{aligned}
\underset{\mathfrak{a} \in \Pi_{\ell}}{\mathbb{E}}\left[\left\|\left\{\mathbf{Y}_{\mathfrak{a}}\right\}-\prod_{i=1}^{\ell}\left\{\mathbf{Y}_{a_{i}}\right\}\right\|_{1}\right] \leq \underset{\{\mathfrak{s}, \mathfrak{t}\} \sim w_{\ell_{1}, \ell_{2}}}{\mathbb{E}}\left[\left\|\left\{\mathbf{Y}_{\mathfrak{s}}, \mathbf{Y}_{\mathfrak{t}}\right\}-\left\{\mathbf{Y}_{\mathfrak{s}}\right\}\left\{\mathbf{Y}_{\mathfrak{t}}\right\}\right\|_{1}\right] \\
+\underset{\mathfrak{s} \sim \Pi_{\ell_{1}}}{\mathbb{E}}\left[\left\|\left\{\mathbf{Y}_{\mathfrak{s}}\right\}-\prod_{i=1}^{\ell_{1}}\left\{\mathbf{Y}_{s_{i}}\right\}\right\|_{1}\right]+\underset{\mathfrak{t} \sim \prod_{\ell_{2}}}{\mathbb{E}}\left[\left\|\left\{\mathbf{Y}_{\mathfrak{t}}\right\}-\prod_{i=1}^{\ell_{2}}\left\{\mathbf{Y}_{t_{i}}\right\}\right\|_{1}\right]
\end{aligned}
$$

One useful observation, is that by using Lemma 6.6 repeatedly, we can reduce the problem of bounding $\mathbb{E}_{\mathfrak{a} \in \Pi_{\ell}}\left\|\left\{\mathbf{Y}_{\mathfrak{a}}\right\}-\prod_{i=1}^{\ell}\left\{\mathbf{Y}_{a_{i}}\right\}\right\|_{1}$ to a problem of bounding

$$
\underset{\{\mathfrak{s}, \mathfrak{t}\} \sim w_{\ell_{1}, \ell_{2}}}{\mathbb{E}}\left\|\left\{\mathbf{Y}_{\mathfrak{s}}, \mathbf{Y}_{\mathfrak{t}}\right\}-\left\{\mathbf{Y}_{\mathfrak{s}}\right\}\left\{\mathbf{Y}_{\mathfrak{t}}\right\}\right\|_{1},
$$

for $\ell_{1}+\ell_{2} \leq k$. Though it is not a direct implication, it is heavily suggested by Fact 2.7 and Theorem 6.2, that if $G_{\ell_{1}, \ell_{2}}$ is a good spectral expander, after an application of Algorithm 6.1 with our chosen parameters, we should be able to bound these expressions. Using a key lemma used from [BRS11], we will prove that this is indeed the case. The only thing we need to make sure after this point, is that the second eigenvalue $\lambda_{2}\left(G_{\ell_{1}, \ell_{2}}\right)$ of the swap graphs $G_{\ell_{1}, \ell_{2}}$ we will be using are small enough for our purposes. Indeed, our choice of $\gamma$ in Theorem 6.3 and Corollary 6.4 is to make sure that the bound we get on $\lambda_{2}\left(G_{\ell_{1}, \ell_{2}}\right)$ from Theorem 5.2 (together with Remark 6.5) is good enough for our purposes. 


\subsection{Breaking Correlations for Expanding CSPs: Proof of Theorem 6.3}

Throughout this section, we will use the somewhat non-standard definition of variance introduced in [BRS11],

$$
\operatorname{Var}\left[\mathbf{Y}_{\mathfrak{a}}\right]=\sum_{\eta \in[q]^{\mathfrak{a}}} \operatorname{Var}\left[\mathbf{1}\left[\mathbf{Y}_{\mathfrak{a}}=\eta\right]\right]
$$

We will use the following central lemma from [BRS11] in our proof of Theorem 6.3:

Lemma 6.7 (Lemma 5.4 from [BRS11]). Let $G=\left(V, E, \Pi_{2}\right)$ be a weighted graph, $\left\{\mathbf{Y}_{1}, \ldots, \mathbf{Y}_{n}\right\}$ a local PSD ensemble, where we have $\operatorname{Supp}\left(\mathbf{Y}_{i}\right) \leq q$ for every $i \in V$, and $q \geq 0$. Suppose $\varepsilon \geq 0$ is a lower bound on the expected statistical difference between independent and correlated sampling along the edges,i.e.,

$$
\varepsilon \leq \underset{\{i, j\} \sim \Pi_{2}}{\mathbb{E}}\left[\left\|\left\{\mathbf{Y}_{i j}\right\}-\left\{\mathbf{Y}_{i}\right\}\left\{\mathbf{Y}_{j}\right\}\right\|_{1}\right]
$$

There exists absolute constants $c_{0} \geq 0$ and $c_{1} \geq 0$ that satisfy the following: If $\lambda_{2}(G) \leq c_{0} \cdot \frac{\varepsilon^{2}}{q^{2}}$. Then, conditioning on a random vertex decreases the variances,

$$
\underset{i \sim \Pi_{1}}{\mathbb{E}} \underset{j \sim \Pi_{2}}{\mathbb{E}} \underset{\left\{\mathbf{Y}_{j}\right\}}{\mathbb{E}}\left[\operatorname{Var}\left[\mathbf{Y}_{i} \mid \mathbf{Y}_{j}\right]\right] \leq \underset{i \sim \Pi_{1}}{\mathbb{E}}\left[\operatorname{Var}\left[\mathbf{Y}_{i}\right]\right]-c_{1} \cdot \frac{\varepsilon^{2}}{q^{2}}
$$

For our applications, we will be instantiating Lemma 6.7 with $G_{\ell_{1}, \ell_{2}}$ as $G$; and with the local PSD ensemble $\left\{\mathbf{Y}_{\mathfrak{a}}\right\}_{\mathfrak{a} \in X}$ that is obtained from $\left\{\mathbf{Y}_{1}, \ldots, \mathbf{Y}_{n}\right\}$ (q.v. Fact 2.7). For convenience, we will write the concrete instance of the Lemma that we will use,

Corollary 6.8. Let $\ell_{1} \geq \ell_{2} \geq 0$ satisfying $\ell_{1}+\ell_{2} \leq k$ be given parameters, and let $G_{\ell_{1}, \ell_{2}}$ be the swap graph defined for a $\gamma-H D X X(\leq k)$. Let $\left\{\mathbf{Y}_{\mathfrak{a}}\right\}_{\mathfrak{a} \in X}$ be a local PSD ensemble; satisfying $\operatorname{Supp}\left(Y_{\mathfrak{a}}\right) \leq q^{k}$ for every $\mathfrak{a} \in X\left(\ell_{1}\right) \cup X\left(\ell_{2}\right)$ for some $q \geq 0$. Suppose $\varepsilon \geq 0$ satisfies,

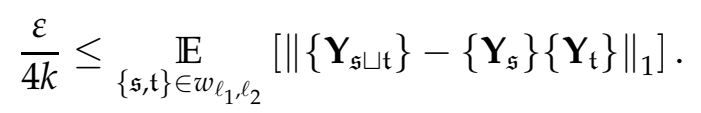

There exists absolute constants $c_{0} \geq 0$ and $c_{2} \geq 0$ that satisfy the following: If $\lambda_{2}(G) \leq c_{0}$. $\left(\varepsilon /\left(4 k \cdot q^{k}\right)\right)^{2}$. Then, conditioning on a random face $\mathfrak{a} \sim \Pi_{\ell_{1}, \ell_{2}}$ decreases the variances, i.e.

$$
\begin{aligned}
2 \cdot \underset{\mathfrak{a}, \mathfrak{b} \sim \Pi_{\ell_{1}, \ell_{2}}^{2}}{\mathbb{E}}\left[\underset{\left\{\mathbf{Y}_{\mathfrak{a}}\right\}}{\mathbb{E}}\left[\operatorname{Var}\left[\mathbf{Y}_{\mathfrak{b}} \mid \mathbf{Y}_{\mathfrak{a}}\right]\right]\right] & =\underset{\mathfrak{a} \in \Pi_{\ell_{1}, \ell_{2}}}{\mathbb{E}}\left[\underset{\mathfrak{s} \sim \Pi_{\ell_{1}}}{\mathbb{E}}\left[\operatorname{Var}\left[\mathbf{Y}_{\mathfrak{s}} \mid \mathbf{Y}_{\mathfrak{a}}\right]\right]+\underset{\mathfrak{t} \sim \Pi_{\ell_{2}}}{\mathbb{E}}\left[\operatorname{Var}\left[\mathbf{Y}_{\mathfrak{t}} \mid \mathbf{Y}_{\mathfrak{a}}\right]\right]\right], \\
& \leq \underset{\mathfrak{s} \sim \Pi_{\ell_{1}}}{\mathbb{E}}\left[\operatorname{Var}\left[\mathbf{Y}_{\mathfrak{s}}\right]\right]+\underset{\mathfrak{t} \sim \Pi_{\ell_{2}}}{\mathbb{E}}\left[\operatorname{Var}\left[\mathbf{Y}_{\mathfrak{t}}\right]\right]-c_{2} \cdot \frac{\varepsilon^{2}}{16 \cdot k^{2} \cdot q^{2 k}} .
\end{aligned}
$$

Here, it can be verified that the expansion criterion presupposed by Lemma 6.7 is satisfied by Corollary 6.8 by Theorem 5.2 . The constant $c_{2}$ satisfies $c_{2}=2 \cdot c_{1}$.

Proof of Theorem 6.3. We will follow the same proof strategy in [BRS11], and extend their arguments for $k$-CSPs.

Write $\Pi_{k}^{m}$ for the distribution of the random set that is obtained in steps (2)-(3) of Algorithm 6.1 with $\Pi=\Pi_{k}$, i.e. $S \sim \Pi_{k}^{m}$ is sampled by

1. independently sampling $m k$-faces $\mathfrak{s}_{j} \sim \Pi_{k}$ for $j=1, \ldots, m$. 
2. outputting $S=\bigcup_{j=1}^{m} \mathfrak{s}_{j}$.

First, for $m \in[L / k]$ we will define

$$
\varepsilon_{m}=\underset{S \sim \prod_{k}^{m}}{\mathbb{E}} \underset{\left\{\mathbf{Y}_{S}\right\}}{\mathbb{E}} \underset{\mathfrak{a} \sim \Pi_{k}}{\mathbb{E}}\left[\left\|\left\{\mathbf{Y}_{\mathfrak{a}} \mid \mathbf{Y}_{S}\right\}-\prod_{j=1}^{k}\left\{\mathbf{Y}_{a_{j}} \mid \mathbf{Y}_{S}\right\}\right\|_{1}\right],
$$

which will measure the average correlation along $X(k)$ after conditioning on $m k$-faces. Notice that our goal is ensuring,

$$
\underset{m \sim[L / k]}{\mathbb{E}} \varepsilon_{m} \leq \varepsilon
$$

where $m$ is sampled uniformly at random.

To help us with this goal, we will define a potential function

$$
\Phi_{m}=\underset{i \sim[k]}{\mathbb{E}} \underset{S \sim \Pi_{k}^{m}}{\mathbb{E}} \underset{\left\{\mathbf{Y}_{S}\right\}}{\mathbb{E}} \underset{\mathfrak{a} \sim \Pi_{i}}{\mathbb{E}} \operatorname{Var}\left[\mathbf{Y}_{\mathfrak{a}} \mid \mathbf{Y}_{S}\right]
$$

where $i$ is sampled uniformly at random. Observe that $\Phi_{m}$ always satisfies $0 \leq \Phi_{m} \leq 1$. Using this, we will try to bound the fraction of indices $m \in[L / k]$ such that $\varepsilon_{m}$ is large, i.e., say $\varepsilon_{m} \geq \varepsilon / 2$. To this end assume $\varepsilon_{m} \geq \varepsilon / 2$, i.e. we have

$$
\underset{S \sim \Pi_{k}^{m}}{\mathbb{E}} \underset{\left\{\mathbf{Y}_{S}\right\}}{\mathbb{E}} \underset{\mathfrak{a} \sim \Pi_{k}}{\mathbb{E}}\left[\left\|\left\{\mathbf{Y}_{\mathfrak{a}} \mid \mathbf{Y}_{S}\right\}-\prod_{i=1}^{k}\left\{\mathbf{Y}_{a_{1}} \mid \mathbf{Y}_{S}\right\}\right\|_{1}\right] \geq \frac{\varepsilon}{2}
$$

We will use Lemma 6.6 in the following way: Let $\mathcal{T}$ be any binary tree with $k$ leaves. We will label each of the vertices $v \in \mathcal{T}$ with the number of leaves of the subtree rooted at $v$. Notice that this ensures that

1. the root vertex of $\mathcal{T}$ has the label $k$,

2. for any vertex $v \in \mathcal{T}$ with label $\ell$, the label $\ell_{1}$ of the left child of $v$ and the label $\ell_{2}$ of the right child of $v$ add up to $k$, i.e. $\ell_{1}+\ell_{2}=k$,

3. every vertex $v \in \mathcal{T}$ with the label 1 is a leaf.

We write $J(\mathcal{T})$ for the set of labels $\ell$ of the internal nodes of $\mathcal{T}$, note $|J(\mathcal{T})| \leq k$. We will use the notation $\ell_{1}$ (resp. $\ell_{2}$ ) to refer to the label of the left (resp. right) of a vertex $v \in \mathcal{T}$ with the label $\ell$.

By applying Lemma 6.6, we obtain that for any local PSD ensemble $\mathbf{Z}$ one has

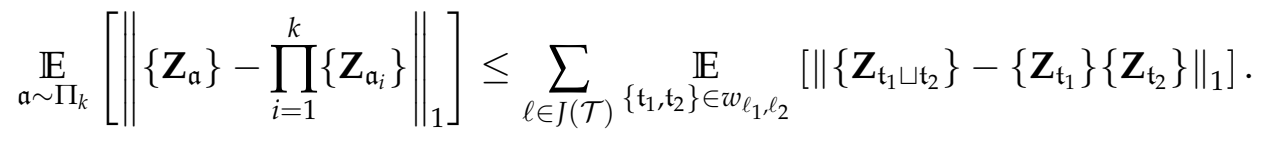

Now, by plugging this in Eq. (13), with $\mathbf{Z}_{\mathfrak{a}}=\left\{\mathbf{Y}_{\mathfrak{a}} \mid \mathbf{Y}_{S}\right\}$, we obtain

$$
\underset{S \sim \prod_{k}^{m}}{\mathbb{E}} \underset{\left\{\mathbf{Y}_{S}\right\}}{\mathbb{E}}\left[\sum_{\ell \in J(\mathcal{T})} \underset{\left\{\mathfrak{t}_{1}, \mathfrak{t}_{2}\right\} \sim w_{\ell_{1}, \ell_{2}}}{\mathbb{E}}\left\|\left\{\mathbf{Y}_{\mathfrak{t}_{1} \sqcup \mathfrak{t}_{2}} \mid \mathbf{Y}_{S}\right\}-\left\{\mathbf{Y}_{\mathfrak{t}_{1}} \mid \mathbf{Y}_{S}\right\}\left\{\mathbf{Y}_{\mathfrak{t}_{2}} \mid \mathbf{Y}_{S}\right\}\right\|_{1}\right] \geq \frac{\varepsilon}{2} .
$$


In particular, in the sum over $J(\mathcal{T})$ there should be some large term corresponding to some $\ell \in J(\mathcal{T})$. i.e. we have,

$$
\underset{S \sim \prod_{k}^{m}}{\mathbb{E}} \underset{\left\{\mathbf{Y}_{S}\right\}}{\mathbb{E}}\left[\underset{\left\{\mathfrak{t}_{1}, \mathfrak{t}_{2}\right\} \in w_{\ell_{1}, \ell_{2}}}{\mathbb{E}}\left\|\left\{\mathbf{Y}_{\mathfrak{t}_{1} \sqcup \mathfrak{t}_{2}} \mid \mathbf{Y}_{S}\right\}-\left\{\mathbf{Y}_{\mathfrak{t}_{1}} \mid \mathbf{Y}_{S}\right\}\left\{\mathbf{Y}_{\mathfrak{t}_{2}} \mid \mathbf{Y}_{S}\right\}\right\|_{1}\right] \geq \frac{\varepsilon}{2 \cdot|J(\mathcal{T})|} \geq \frac{\varepsilon}{2 k} .
$$

Now, we have

$$
\underset{\substack{S \sim \Pi_{k}^{m} \\\left\{\mathbf{Y}_{S}\right\}}}{\mathbb{P}}\left[\underset{\{\mathfrak{s}, \mathfrak{t}\} \in w_{\ell_{1}, \ell_{2}}}{\mathbb{E}}\left\|\left\{\mathbf{Y}_{\mathfrak{t}_{1} \sqcup \mathfrak{t}_{2}} \mid \mathbf{Y}_{S}\right\}-\left\{\mathbf{Y}_{\mathfrak{t}_{1}} \mid \mathbf{Y}_{S}\right\}\left\{\mathbf{Y}_{\mathfrak{t}_{2}} \mid \mathbf{Y}_{S}\right\}\right\|_{1} \geq \frac{\varepsilon}{4 k}\right] \geq \frac{\varepsilon}{4 k}
$$

This together with Corollary 6.8 implies,

$$
\begin{array}{r}
\underset{\substack{S \sim \Pi_{k}^{m} \\
\left\{\mathbf{Y}_{S}\right\}}}{\mathbb{P}}\left[\underset{\mathfrak{a} \in \Pi_{\ell_{1}, \ell_{2}}}{\mathbb{E}}\left[\begin{array}{c}
\mathbb{E}_{\mathfrak{t}_{1} \sim \Pi_{\ell_{1}}}\left[\operatorname{Var}\left[\mathbf{Y}_{\mathfrak{t}_{1}} \mid \mathbf{Y}_{S}\right]-\operatorname{Var}\left[\mathbf{Y}_{\mathfrak{t}_{1}} \mid \mathbf{Y}_{S}, \mathbf{Y}_{\mathfrak{a}}\right]\right] \\
+\quad \mathbb{E}_{\mathfrak{t}_{2} \in \Pi_{\ell_{2}}}\left[\operatorname{Var}\left[\mathbf{Y}_{\mathfrak{t}_{2}}|| \mathbf{Y}_{S}\right]-\operatorname{Var}\left[\mathbf{Y}_{\mathfrak{t}_{2}} \mid \mathbf{Y}_{S}, \mathbf{Y}_{\mathfrak{a}}\right]\right]
\end{array}\right] \geq c_{2} \cdot \frac{\varepsilon^{2}}{16 \cdot k^{2} \cdot q^{2 k}}\right] \\
\geq \frac{\varepsilon}{4 k},
\end{array}
$$

provided that $\lambda_{2}\left(G_{\ell_{1}, \ell_{2}}\right) \leq c_{0}\left(\varepsilon /\left(4 k \cdot q^{k}\right)\right)^{2}$.

Now, observe that a sample $\mathfrak{a} \sim \Pi_{\ell_{1}, \ell_{2}}$ can be obtained from a sample $\mathfrak{s}_{m+1} \sim \Pi_{k}$ in the following way,

1. with probability $\frac{1}{2}$ each, pick $j=1$ or $j=2$.

2. delete all but $\ell_{j}$ elements from $\mathfrak{s}_{m+1}$.

It is important to note that for the sample $\mathfrak{a} \sim \Pi_{\ell_{1}, \ell_{2}}$ obtained this way, we have $\mathfrak{s}_{m+1} \supseteq$ $\mathfrak{a}$. An application of Jensen's inequality shows that the variance is non-increasing under conditioning, i.e. for random variables $\mathbf{Z}$ and $\mathbf{W}$ we have,

$$
\begin{aligned}
\underset{\mathbf{Z}}{\mathbb{E}}[\operatorname{Var}[\mathbf{W} \mid \mathbf{Z}]] & =\underset{\mathbf{Z}}{\mathbb{E}}\left[\underset{\mathbf{W}}{\mathbb{E}}\left[\mathbf{W}^{2} \mid \mathbf{Z}\right]\right]-\underset{\mathbf{Z}}{\mathbb{E}}\left[\left(\underset{\mathbf{W}}{\mathbb{E}}[\mathbf{W} \mid \mathbf{Z}]^{2}\right)\right] \\
& \leq \mathbb{E}\left[\mathbf{W}^{2}\right]-(\underset{\mathbf{Z}}{\mathbb{E}}[\mathbb{E}[\mathbf{W} \mid \mathbf{Z}]])^{2} \\
& =\operatorname{Var}[\mathbf{W}] .
\end{aligned}
$$

This means conditioning on $\mathfrak{s}_{m+1}$, the drop in variance can only be more, i.e., Eq. (15) implies

$$
\underset{\substack{s \sim \Pi_{k}^{m} \\
\left\{\mathbf{Y}_{S}\right\}}}{\mathbb{P}}\left[\underset{\mathfrak{s}_{m+1} \in \Pi_{k}}{\mathbb{E}}\left[\begin{array}{c}
\mathbb{E}_{\mathfrak{t}_{1} \sim \Pi_{\ell_{1}}}\left[\operatorname{Var}\left[\mathbf{Y}_{\mathfrak{t}_{1}} \mid \mathbf{Y}_{S}\right]-\operatorname{Var}\left[\mathbf{Y}_{\mathfrak{t}_{1}} \mid \mathbf{Y}_{S}, \mathbf{Y}_{\mathfrak{s}_{m+1}}\right]\right] \\
+\quad \mathbb{E}_{\mathfrak{t}_{2} \in \Pi_{\ell_{2}}}\left[\operatorname{Var}\left[\mathbf{Y}_{\mathfrak{t}_{2}}|| \mathbf{Y}_{S}\right]-\operatorname{Var}\left[\mathbf{Y}_{\mathfrak{t}_{2}} \mid \mathbf{Y}_{S}, \mathbf{Y}_{\mathfrak{s}_{m+1}}\right]\right]
\end{array}\right] \geq c_{2} \cdot \frac{\varepsilon^{2}}{16 \cdot k^{2} \cdot q^{2 k}}\right] \geq \frac{\varepsilon}{4 k}
$$

By relabeling $\ell_{1}$ as $\ell_{2}$ if needed, we can obtain the following inequality from the above

$$
\underset{\substack{s \sim \Pi_{k}^{m} \\\left\{\mathbf{Y}_{S}\right\}}}{\mathbb{P}}\left[\underset{\mathfrak{s}_{m+1} \in \Pi_{k}}{\mathbb{E}}\left[\underset{\mathfrak{t}_{1} \sim \Pi_{\ell_{1}}}{\mathbb{E}}\left[\operatorname{Var}\left[\mathbf{Y}_{\mathfrak{t}_{1}} \mid \mathbf{Y}_{S}\right]-\operatorname{Var}\left[\mathbf{Y}_{\mathfrak{t}_{1}} \mid \mathbf{Y}_{S}, \mathbf{Y}_{\mathfrak{s}_{m+1}}\right]\right]\right] \geq c_{2} \cdot \frac{\varepsilon^{2}}{32 \cdot k^{2} \cdot q^{2 k}}\right] \geq \frac{\varepsilon}{4 k}
$$


This implies

$$
\Phi_{m}-\Phi_{m+1} \geq \frac{1}{k} \cdot \frac{\varepsilon}{4 k} \cdot\left(c_{2} \cdot \frac{\varepsilon^{2}}{32 \cdot k^{2} \cdot q^{2 k}}\right)=c_{2} \cdot \frac{\varepsilon^{3}}{128 \cdot k^{4} \cdot q^{2 k}}
$$

where the $\frac{1}{k}$ term in the RHS corresponds to $\ell_{1} \in[k]$ being chosen in Eq. (12), the $\frac{\varepsilon}{4 k}$ term in the RHS corresponds to the probability of the variances in $X\left(\ell_{1}\right)$ drop by $\left(c_{2} \cdot \frac{\varepsilon^{2}}{32 \cdot k^{2} \cdot q^{k}}\right)$. Since, the variance is non-increasing under conditioning

$$
1 \geq \Phi_{1} \geq \cdots \geq \Phi_{m} \geq 0
$$

this means there can be at most $128 k^{4} \cdot q^{2 k} /\left(c_{2} \cdot \varepsilon^{3}\right)$ indices $m \in[L / k]$ such that $\varepsilon_{m} \geq \varepsilon / 2$. In particular, since the total number of indices is $(L / k)$ we have,

$$
\underset{m \sim[L / k]}{\mathbb{E}} \varepsilon_{m} \leq \frac{\varepsilon}{2}+\frac{k}{L} \cdot \frac{128 \cdot k^{4} \cdot q^{2 k}}{c_{2} \cdot \varepsilon^{3}}
$$

This means that there exists an absolute constant $c^{\prime} \geq 0$ such that

$$
L \geq c^{\prime} \cdot \frac{k^{5} \cdot q^{2 k}}{\varepsilon^{4}} \quad \text { ensures } \underset{m \in[L / k]}{\mathbb{E}}\left[\varepsilon_{m}\right] \leq \varepsilon
$$

To finish our proof, we note that to justify our applications of Corollary 6.8 it suffices to ensure

$$
\lambda_{2}\left(G_{\ell_{1}, \ell_{2}}\right) \leq c_{0} \cdot\left(\frac{\varepsilon}{4 k \cdot q^{k}}\right)^{2}=c_{0} \cdot \frac{\varepsilon^{2}}{16 \cdot k^{2} \cdot q^{2 k}}
$$

for all $\ell_{1}, \ell_{2}$ occurring in $\mathcal{T}$ as a label. It can be verified that our choice of $\gamma$ together with Theorem 5.2 (and Remark 6.5) satisfies this, where the constant $C^{\prime} \geq 0$ will account for $c_{0}$, $c^{\prime}$, and the constants hidden within the O-notation in Theorem 5.2.

\subsection{The Glorified Triangle Inequality: Proof of Lemma 6.6}

In this Section, we will prove Lemma 6.6.

Proposition 6.9. Let $\mathbf{Y}, \mathbf{Z}, \mathbf{U}, \mathbf{W}$ be random variables where $\mathbf{Y}$ and $\mathbf{Z}$; and $\mathbf{U}$ and $\mathbf{W}$ are on the same support. Then,

$$
\|\{\mathbf{Y}\}\{\mathbf{U}\}-\{\mathbf{Z}\}\{\mathbf{W}\}\|_{1} \leq\|\{\mathbf{Y}\}-\{\mathbf{Z}\}\|_{1}+\|\{\mathbf{U}\}-\{\mathbf{W}\}\|_{1} .
$$

Proof. Tensoring with the same probability distribution does not change the total variation distance, i.e.

$\|\{\mathbf{Y}\}-\{\mathbf{Z}\}\|_{1}=\|\{\mathbf{Y}\}\{\mathbf{U}\}-\{\mathbf{Z}\}\{\mathbf{U}\}\|_{1} \quad$ and $\quad\|\{\mathbf{U}\}-\{\mathbf{W}\}\|_{1}=\|\{\mathbf{Z}\}\{\mathbf{U}\}-\{\mathbf{Z}\}\{\mathbf{W}\}\|_{1}$.

Now, a simple application of the triangle inequality proves the Proposition.

A straightforward implication of Proposition 6.9 is the following, which will allow us to bound the correlation along a face $\mathfrak{a} \in X(k)$, using the correlation along sub-faces $\mathfrak{s ,} \mathfrak{t} \subseteq \mathfrak{a}$. 
Corollary 6.10. Let $\mathfrak{a} \in X(\ell)$ and $\mathfrak{s} \in X\left(\ell_{1}\right), \mathfrak{t} \in X\left(\ell_{2}\right)$ be given such that $\mathfrak{a}=\mathfrak{s} \sqcup \mathfrak{t}$. Then for any $k$-local PSD ensemble $\left\{\mathbf{Y}_{1}, \ldots, \mathbf{Y}_{n}\right\}$ we have

$$
\begin{aligned}
\left\|\left\{\mathbf{Y}_{\mathfrak{a}}\right\}-\left\{\mathbf{Y}_{a_{1}}\right\} \cdots\left\{\mathbf{Y}_{a_{\ell}}\right\}\right\|_{1} & \leq\left\|\left\{\mathbf{Y}_{\mathfrak{a}}\right\}-\left\{\mathbf{Y}_{\mathfrak{s}}\right\}\left\{\mathbf{Y}_{\mathfrak{t}}\right\}\right\|_{1} \\
& +\left\|\left\{\mathbf{Y}_{\mathfrak{s}}\right\}-\left\{\mathbf{Y}_{s_{1}}\right\} \cdots\left\{\mathbf{Y}_{s_{\ell_{1}}}\right\}\right\|_{1}+\left\|\left\{\mathbf{Y}_{\mathfrak{t}}\right\}-\left\{\mathbf{Y}_{t_{1}}\right\} \cdots\left\{\mathbf{Y}_{t_{\ell_{2}}}\right\}\right\|_{1}
\end{aligned}
$$

With this, we can go ahead and prove Lemma 6.6

Proof of Lemma 6.6. Let $\mathfrak{a} \in X(\ell)$ be a fixed face. By Corollary 6.10 and averaging over all the $\left(\begin{array}{c}\ell=\ell_{1}+\ell_{2} \\ \ell_{1}\end{array}\right)$ ways of splitting $\mathfrak{a}$ into $\{\mathfrak{s}, \mathfrak{t}\}$ such that $\mathfrak{s} \in X\left(\ell_{1}\right)$ and $\mathfrak{t} \in X\left(\ell_{2}\right)$ we have

$$
\begin{aligned}
& \left\|\left\{\mathbf{Y}_{\mathfrak{a}}\right\}-\prod_{i=1}^{\ell=\ell_{1}+\ell_{2}}\left\{\mathbf{Y}_{a_{i}}\right\}\right\|_{1} \\
& \quad \leq \frac{1}{\left(\begin{array}{c}
\ell_{1}+\ell_{2} \\
\ell_{1}
\end{array}\right)} \sum_{\{\mathfrak{s}, \mathfrak{t}\}}\left(\left\|\left\{\mathbf{Y}_{\mathfrak{a}}\right\}-\left\{\mathbf{Y}_{\mathfrak{s}}\right\}\left\{\mathbf{Y}_{\mathfrak{t}}\right\}\right\|_{1}+\left\|\left\{\mathbf{Y}_{\mathfrak{s}}\right\}-\prod_{i=1}^{\ell_{1}}\left\{Y_{s_{i}}\right\}\right\|_{1}+\left\|\left\{\mathbf{Y}_{\mathfrak{t}}\right\}-\prod_{i=1}^{\ell_{2}}\left\{\mathbf{Y}_{t_{i}}\right\}\right\|\right) .
\end{aligned}
$$

Now, by taking an average over all the edges $\mathfrak{a} \in X(\ell)$ (with respect to the measure $\Pi_{\ell}$ ) we obtain,

$$
\begin{aligned}
\underset{\mathfrak{a} \sim \Pi_{\ell}}{\mathbb{E}}\left[\left\|\left\{\mathbf{Y}_{\mathfrak{a}}\right\}-\prod_{i=1}^{\ell}\left\{\mathbf{Y}_{a_{i}}\right\}\right\|_{1}\right] \\
\leq \frac{1}{\left(\begin{array}{c}
\ell \\
\ell_{1}
\end{array}\right)} \cdot \underset{\mathfrak{a} \in \Pi_{\ell}}{\mathbb{E}}\left[\sum_{\{\mathfrak{s}, \mathfrak{t}\}}\left(\left\|\left\{\mathbf{Y}_{\mathfrak{a}}\right\}-\left\{\mathbf{Y}_{\mathfrak{s}}\right\}\left\{\mathbf{Y}_{\mathfrak{t}}\right\}\right\|_{1}+\left\|\left\{\mathbf{Y}_{\mathfrak{s}}\right\}-\prod_{i=1}^{k_{1}}\left\{\mathbf{Y}_{s_{i}}\right\}\right\|_{1}+\left\|\left\{\mathbf{Y}_{\mathfrak{t}}\right\}-\prod_{i=1}^{\ell_{2}}\left\{\mathbf{Y}_{t_{i}}\right\}\right\|_{1}\right)\right]
\end{aligned}
$$

where the indices $\{\mathfrak{s}, \mathfrak{t}\}$ run over the all the ways of splitting $\mathfrak{a}$ into $\mathfrak{s}$ and $\mathfrak{t}$ as before. We can now see that the RHS can be thought as an average over the (weighted) edges in $E\left(\ell_{1}, \ell_{2}\right)$ (q.v. Eq. (10)), i.e.,

$$
\begin{aligned}
& \underset{\mathfrak{a} \sim \prod_{\ell}}{\mathbb{E}}\left[\left\|\left\{\mathbf{Y}_{\mathfrak{a}}\right\}-\prod_{i=1}^{\ell}\left\{\mathbf{Y}_{a_{i}}\right\}\right\|_{1}\right] \\
& \leq \underset{\{\mathfrak{s}, \mathfrak{t}\} \sim w_{\ell_{1}, \ell_{2}}}{\mathbb{E}}\left[\left\|\left\{\mathbf{Y}_{\mathfrak{a}}\right\}-\left\{\mathbf{Y}_{\mathfrak{s}}\right\}\left\{\mathbf{Y}_{\mathfrak{t}}\right\}\right\|_{1}+\left\|\left\{\mathbf{Y}_{\mathfrak{s}}\right\}-\prod_{i=1}^{\ell_{1}}\left\{\mathbf{Y}_{s_{i}}\right\}\right\|_{1}+\left\|\left\{\mathbf{Y}_{\mathfrak{t}}\right\}-\prod_{i=1}^{\ell_{2}}\left\{Y_{t_{i}}\right\}\right\|_{1}\right]
\end{aligned}
$$

Now, note that since $\Pi_{\ell_{1}, \ell_{2}}$ (q.v. Eq. (9)) is the stationary distribution of the walk defined on $G_{\ell_{1}, \ell_{2}}$, i.e.,

$$
2 \Pi_{\ell_{1}, \ell_{2}}(\mathfrak{a})=\sum_{\mathfrak{b}:\{\mathfrak{a}, \mathfrak{b}\} \in E\left(\ell_{1}, \ell_{2}\right)} w_{\ell_{1}, \ell_{2}}(\mathfrak{a}, \mathfrak{b}),
$$

the lemma follows. This is because, we have

$$
\begin{aligned}
& \underset{\mathfrak{a} \in X(\ell)}{\mathbb{E}}\left[\left\|\left\{\mathbf{Y}_{\mathfrak{a}}\right\}-\prod_{i=1}^{\ell}\left\{\mathbf{Y}_{a_{i}}\right\}\right\|_{1}\right] \\
& \leq \underset{\{\mathfrak{s}, \mathfrak{t}\} \sim w_{\ell_{1}, \ell_{2}}}{\mathbb{E}}\left[\left\|\left\{\mathbf{Y}_{\mathfrak{a}}\right\}-\left\{\mathbf{Y}_{\mathfrak{s}}\right\}\left\{\mathbf{Y}_{\mathfrak{t}}\right\}\right\|_{1}\right]+\underset{\{\mathfrak{s}, \mathfrak{t}\} \sim w_{\ell_{1}, \ell_{2}}}{\mathbb{E}}\left[\left\|\left\{\mathbf{Y}_{\mathfrak{s}}\right\}-\prod_{i=1}^{\ell_{1}}\left\{\mathbf{Y}_{s_{i}}\right\}\right\|_{1}+\left\|\left\{\mathbf{Y}_{\mathfrak{t}}\right\}-\prod_{i=1}^{\ell_{2}}\left\{Y_{t_{i}}\right\}\right\|_{1}\right] \\
& =\underset{\{\mathfrak{s}, \mathfrak{t}\} \sim E\left(\ell_{1}, \ell_{2}\right)}{\mathbb{E}}\left[\left\|\left\{\mathbf{Y}_{\mathfrak{a}}\right\}-\left\{\mathbf{Y}_{\mathfrak{s}}\right\}\left\{\mathbf{Y}_{\mathfrak{t}}\right\}\right\|_{1}\right]+\underset{\mathfrak{s} \sim \Pi_{\ell_{1}}}{\mathbb{E}}\left[\left\|\left\{\mathbf{Y}_{\mathfrak{s}}\right\}-\prod_{i=1}^{\ell_{1}}\left\{\mathbf{Y}_{s_{i}}\right\}\right\|_{1}\right]+\underset{\mathfrak{t} \sim \Pi_{\ell_{2}}}{\mathbb{E}}\left[\left\|\left\{\mathbf{Y}_{\mathfrak{t}}\right\}-\prod_{i=1}^{\ell_{2}}\left\{\mathbf{Y}_{t_{i}}\right\}\right\|_{1}\right]
\end{aligned}
$$




\section{High-Dimensional Threshold Rank}

In [BRS11], Theorem 6.2 was proven for a more general class of graphs than expander graphs - namely, the class of low threshold rank graphs.

Definition 7.1 (Threshold Rank of Graphs (from [BRS11])). Let $G=(V, E, w)$ be a weighted graph on $n$ vertices and $\mathrm{A}$ be its normalized random walk matrix. Suppose the eigenvalues of $\mathrm{A}$ are $1=\lambda_{1} \geq \cdots \geq \lambda_{n}$. Given a parameter $\tau \in(0,1)$, we denote the threshold rank of $G$ by $\operatorname{rank}_{\geq \tau}(\mathrm{A})$ (or $\operatorname{rank}_{\geq \tau}(G)$ ) and define it as

$$
\operatorname{rank}_{\geq \tau}(\mathrm{A}):=\left|\left\{i \mid \lambda_{i} \geq \tau\right\}\right|
$$

There [BRS11], the authors asked for the correct notion of threshold rank for $k$-CSPs. In this section, we give a candidate definition of low threshold rank motivated by our techniques.

To break $k$-wise correlations it is sufficient to assume that the involved swap graphs in the foregoing discussion are low threshold rank since this is enough to apply a version of Lemma 6.7, already described in the work of [BRS11].

Moreover, we have some flexibility as to which swap graphs to consider as long as they satisfy some splitting conditions. To define a swap graph it is enough to have a distributions on the hyperedges of a (constraint) hypergraph. Hence, the notion of swap graph is independent of high-dimensional expansion. HDXs are just an interesting family of objects for which the swap graphs are good expanders.

To capture the many ways of splitting the statistical distance over hyperedges into the statistical distance over the edges of swap graphs, we first define the following notion. We say that a binary tree $\mathcal{T}$ is a $k$-splitting tree if it has exactly $k$ leaves. Thus, labeling every vertex with the number of leaves on the subtree rooted at that vertex ensures,

- the root of $\mathcal{T}$ is labeled with $k$ and all other vertices are labeled with positive integers,

- the leaves are labeled with 1 , and

- each non-leaf vertex satisfy the property that its label is the sum of the labels of its two children.

Note that, we will think of each non-leaf node with left and right children labeled as $a$ and $b$ as representing the swap graph from $X(a)$ to $X(b)$ for some simplicial complex $X(\leq k)$. Let $\operatorname{Swap}(\mathcal{T}, X)$ be the set of all such swap graphs over $X$ finding representation in the splitting tree $\mathcal{T}$. Indeed the tree $\mathcal{T}$ used in the proof of Theorem 6.3 is just one special instance of a $k$-splitting tree.

Given a threshold parameter $\tau \leq 1$ and a set of normalized adjacency matrices $\mathcal{A}=$ $\left\{\mathrm{A}_{1}, \ldots, \mathrm{A}_{s}\right\}$, we define the threshold rank of $\mathcal{A}$ as

$$
\operatorname{rank}_{\geq \tau}(\mathcal{A}):=\max _{\mathrm{A} \in \mathcal{A}} \operatorname{rank}_{\geq \tau}(\mathrm{A}),
$$

where $\operatorname{rank}_{\geq \tau}(A)$ is denotes usual threshold rank of $A$ as in Definition 7.1.

Now, we are ready to define the notion of a $k$-CSP instance being $(\mathcal{T}, \tau, r)$-splittable as follows. 
Definition 7.2 $((\mathcal{T}, \tau, r)$-splittability). A $k$-CSP instance $\mathfrak{I}$ with the constraint complex $X(\leq k)$ is said to be $(\mathcal{T}, \tau, r)$-splittable if $\mathcal{T}$ is a $k$-splitting tree and

$$
\operatorname{rank}_{\geq \tau}(\operatorname{Swap}(\mathcal{T}, X)) \leq r .
$$

If there exists some $k$-splitting tree $\mathcal{T}$ such that $\mathfrak{I}$ is $(\mathcal{T}, \tau, r)$-splittable, the instance $\mathfrak{I}$ will be called $a(\tau, r)$-splittable instance.

Now, using this definition we can show that whenever $\operatorname{rank}_{\tau}(\mathfrak{I})$ is bounded for the appropriate choice of $\tau$, after conditioning on a random partial assignment as in Algorithm 6.1 we will have small correlation over the faces $\mathfrak{a} \in X(k)$, i.e.,

Theorem 7.3. Suppose a simplicial complex $X(\leq k)$ with $X(1)=[n]$ and an L-local PSD ensemble $\mathbf{Y}=\left\{\mathbf{Y}_{1}, \ldots, \mathbf{Y}_{n}\right\}$ are given. There exists some universal constants $c_{4} \geq 0$ and $C^{\prime \prime} \geq 0$ satisfying the following: If $L \geq C^{\prime \prime} \cdot\left(q^{4 k} \cdot k^{7} \cdot r / \varepsilon^{5}\right), \operatorname{Supp}\left(\mathbf{Y}_{j}\right) \leq q$ for all $j \in[n]$, and $\mathfrak{I}$ is $\left(c_{4} \cdot\left(\varepsilon /\left(4 k \cdot q^{k}\right)\right)^{2}, r\right)$-splittable. Then, we have

$$
\underset{\mathfrak{a} \in X(k)}{\mathbb{E}}\left[\left\|\left\{\mathbf{Y}_{\mathfrak{a}}^{\prime}\right\}-\left\{\mathbf{Y}_{a_{1}}^{\prime}\right\} \cdots\left\{\mathbf{Y}_{a_{k}}^{\prime}\right\}\right\|_{1}\right] \leq \varepsilon,
$$

where $\mathbf{Y}^{\prime}$ is as defined in Algorithm 6.1 on the input of $\left\{\mathbf{Y}_{1}, \ldots, \mathbf{Y}_{n}\right\}$ and $\Pi_{k}$.

It is important to note that the specific knowledge of the $k$-splitting tree $\mathcal{T}$ that makes $\mathfrak{I}$ $(\mathcal{T}, \tau, r)$-splittable is only needed for the proof of Theorem 7.3. The conclusion of Theorem 7.3 can be used without the knowledge of the specific $k$-splitting tree $\mathcal{T}$. The attentive reader might have noticed is that in the proof of Theorem 6.3, the choice of $\mathcal{T}$ is not important, as all the splitting tree are guaranteed to have be expanders provided that $X$ is a $\gamma$-HDX. The proof of Theorem 7.3, in this light can be thought of an extension of the proof of Theorem 6.3 to the case where not necessarily every tree is good, and where we can bound the threshold rank instead of the spectral expansion.

This, will readily imply an algorithm

Corollary 7.4. Suppose $\mathfrak{I}$ is a q-ary $k$-CSP instance whose constraint complex is $X(\leq k)$. There exists an absolute constant $C^{\prime \prime} \geq 0$ and $c_{4} \geq 0$ that satisfies the following: If $\mathfrak{I}$ is $\left(c_{4} \cdot(\varepsilon /(4 k\right.$. $\left.\left.\left.q^{k}\right)\right)^{2}, r\right)$-splittable, then there is an algorithm that runs in time $n^{O\left(\frac{q^{4 k} \cdot k^{7} \cdot r}{\varepsilon^{5}}\right)}$ and that is based on $\left(\frac{C^{\prime \prime} \cdot k^{5} \cdot q^{k} \cdot r}{\varepsilon^{4}}\right)$-levels of SoS-hierarchy and Algorithm 6.1 that outputs a random assignment $\eta:[n] \rightarrow$ $[q]$ that in expectation ensures $\operatorname{SAT}_{\mathfrak{I}}(\eta)=\mathrm{OPT}(\mathfrak{I})-\varepsilon$.

Since the proof of Corollary 7.4 given Theorem 7.3, will be almost identical to the proof of Corollary 6.4, given Theorem 6.3, we will omit the proof of this.

\subsection{Breaking Correlations for Splittable CSPs: Proof of Theorem 7.3}

We will need the more general version of Lemma 6.7, already proven in [BRS11].

Lemma 7.5 (Lemma 5.4 from [BRS11]). ${ }^{7}$ Let $G=\left(V, E, \Pi_{2}\right)$ be a weighted graph, $\left\{\mathbf{Y}_{1}, \ldots, \mathbf{Y}_{n}\right\}$ a local PSD ensemble, where we have $\operatorname{Supp}\left(\mathbf{Y}_{i}\right) \leq q$ for every $i \in V$, and $q \geq 0$. If $\varepsilon \geq 0$ is a lower

\footnotetext{
${ }^{7}$ We give a derivation of this lemma in Appendix A.
} 
bound on the expected statistical difference between independent and correlated sampling along the edges,i.e.,

$$
\varepsilon \leq \underset{\{i, j\} \sim \Pi_{2}}{\mathbb{E}}\left[\left\|\left\{\mathbf{Y}_{i j}\right\}-\left\{\mathbf{Y}_{i}\right\}\left\{\mathbf{Y}_{j}\right\}\right\|_{1}\right]
$$

There exists absolute constants $c_{3} \geq 0$ and $c_{4} \geq 0$ that satisfy the following: Then, conditioning on a random vertex decreases the variances,

$$
\underset{i \sim \Pi_{1}}{\mathbb{E}} \underset{j \sim \Pi_{1}}{\mathbb{E}} \underset{\left\{\mathbf{Y}_{j}\right\}}{\mathbb{E}}\left[\operatorname{Var}\left[\mathbf{Y}_{i} \mid \mathbf{Y}_{j}\right]\right] \leq \underset{i \sim \Pi_{1}}{\mathbb{E}}\left[\operatorname{Var}\left[\mathbf{Y}_{i}\right]\right]-c_{3} \cdot \frac{\varepsilon^{4}}{q^{4} \cdot \operatorname{rank}_{\geq c_{4} \varepsilon^{2} / q^{2}}(G)}
$$

Since we will use this lemma, only with the swap graphs $G_{\ell_{1}, \ell_{2}}$ and $(L / k)$-local PSD ensemble $\left\{\mathbf{Y}_{\mathfrak{a}}\right\}_{\mathfrak{a} \in X}$ obtained from the $L$-local PSD ensemble $\left\{\mathbf{Y}_{1}, \ldots, \mathbf{Y}_{n}\right\}$, for convenience we will write the corollary we will use more explicitly

Corollary 7.6. Let $\ell_{1} \geq \ell_{2} \geq 0$ satisfying $\ell_{1}+\ell_{2} \leq k$ be given parameters, and let $G_{\ell_{1}, \ell_{2}}$ be the swap graph defined for a $\gamma$-HDX $X(\leq k)$. Let $\left\{\mathbf{Y}_{\mathfrak{a}}\right\}_{\mathfrak{a} \in X}$ be a local PSD ensemble; and suppose we have $\operatorname{Supp}\left(Y_{\mathfrak{a}}\right) \leq q^{k}$ for every $\mathfrak{a} \in X\left(\ell_{1}\right) \cup X\left(\ell_{2}\right)$ for some $q \geq 0$. Suppose $\varepsilon>0$ satisfies,

$$
\frac{\varepsilon}{4 k} \leq \underset{\{\mathfrak{s}, \mathfrak{t}\} \in E\left(\ell_{1}, \ell_{2}\right)}{\mathbb{E}}\left[\left\|\left\{\mathbf{Y}_{\mathfrak{s} \cup \mathfrak{t}}\right\}-\left\{\mathbf{Y}_{\mathfrak{s}}\right\}\left\{\mathbf{Y}_{\mathfrak{t}}\right\}\right\|_{1}\right]
$$

There exists absolute constants $c_{3} \geq 0$ and $c_{5} \geq 0$ that satisfy the following:

If $\operatorname{rank}_{\geq c_{4} \cdot\left(\varepsilon /\left(4 k \cdot q^{k}\right)\right)^{2}}\left(G_{\ell_{1}, \ell_{2}}\right) \leq r$, then conditioning on a random face $\mathfrak{a} \sim \Pi_{\ell_{1}, \ell_{2}}$ decreases the variances, i.e.

$$
\begin{aligned}
2 \cdot \underset{\mathfrak{a}, \mathfrak{b} \sim \Pi_{\ell_{1}, \ell_{2}}}{\mathbb{E}}\left[\underset{\left\{\mathbf{Y}_{\mathfrak{a}}\right\}}{\mathbb{E}}\left[\operatorname{Var}\left[\mathbf{Y}_{\mathfrak{b}} \mid \mathbf{Y}_{\mathfrak{a}}\right]\right]\right] & =\underset{\mathfrak{a} \in \Pi_{\ell_{1}, \ell_{2}}}{\mathbb{E}}\left[\underset{\mathfrak{s} \sim \Pi_{\ell_{1}}}{\mathbb{E}}\left[\operatorname{Var}\left[\mathbf{Y}_{\mathfrak{s}} \mid \mathbf{Y}_{\mathfrak{a}}\right]\right]+\underset{\mathfrak{t} \sim \Pi_{\ell_{2}}}{\mathbb{E}}\left[\operatorname{Var}\left[\mathbf{Y}_{\mathfrak{t}} \mid \mathbf{Y}_{\mathfrak{a}}\right]\right]\right] \\
& \leq \underset{\mathfrak{s} \sim \Pi_{\ell_{1}}}{\mathbb{E}}\left[\operatorname{Var}\left[\mathbf{Y}_{\mathfrak{s}}\right]\right]+\underset{\mathfrak{t} \sim \Pi_{\ell_{2}}}{\mathbb{E}}\left[\operatorname{Var}\left[\mathbf{Y}_{\mathfrak{t}}\right]\right]-c_{5} \cdot \frac{\varepsilon^{4}}{256 \cdot k^{4} \cdot q^{4 k} \cdot r}
\end{aligned}
$$

Here the constant $c_{5}$ satisfies $c_{5}=2 \cdot c_{3}$.

Proof. As the proof will mostly follow Theorem 6.3, we will only highlight the relevant differences and carry out the relevant computations.

Let $\tau=c_{4} \cdot\left(\varepsilon /\left(4 k \cdot q^{k}\right)\right)^{2}$, and let $\mathcal{T}$ be the $k$-splitting tree certifying that $\mathfrak{I}$ is $(\mathcal{T}, \tau, r)$ splittable, i.e., the tree $\mathcal{T}$ satisfies $\operatorname{rank}_{\tau}(\operatorname{Swap}(\mathcal{T}, X)) \leq r$. This means that all the swap graphs $G_{\ell_{1}, \ell_{2}}$ finding representation in $\mathcal{T}$ satisfy $\operatorname{rank}_{\tau}\left(G_{\ell_{1}, \ell_{2}}\right) \leq r$.

Similarly, as in the proof of we will try to argue that the fraction of indices $m \in[L / k]$ such that $\varepsilon_{m}$ that is large, say $\varepsilon_{m} \geq \varepsilon / 2$, is small by arguing about the potential $\Phi_{m}$ with both quantities $\varepsilon_{m}$ and $\Phi_{m}$ as defined as in the Proof of Theorem 6.3. We assume similarly, that $\varepsilon_{m} \geq \varepsilon / 2$ for some $m \in[L / k]$.

Analogously to Section 7.1 in the proof of Theorem 6.3, from Corollary 7.6 we obtain

$$
\underset{S \sim \Pi_{k}^{m}}{\mathbb{E}} \underset{\left\{\mathbf{Y}_{S}\right\}}{\mathbb{E}}\left[\sum_{\ell \in J(\mathcal{T})} \underset{\left\{\mathfrak{t}_{1}, \mathfrak{t}_{2}\right\} \in E\left(\ell_{1}, \ell_{2}\right)}{\mathbb{E}}\left[\left\|\left\{\mathbf{Y}_{\mathfrak{t}_{1} \sqcup \mathfrak{t}_{2}} \mid \mathbf{Y}_{S}\right\}-\left\{\mathbf{Y}_{\mathfrak{t}_{1}} \mid \mathbf{Y}_{S}\right\}\left\{\mathbf{Y}_{\mathfrak{t}_{2}} \mid \mathbf{Y}_{S}\right\}\right\|_{1}\right]\right] \geq \frac{\varepsilon}{2}
$$

Notice that the assumption that Section 7.1 makes on the threshold rank is satisfied by the assumption $\operatorname{rank}_{\tau}(\mathfrak{I}) \leq r$ and where the set $J(\mathcal{T})$ contains all labels $\ell$ of internal nodes 
$v \in \mathcal{T}$, and we write $\ell_{1}$ (resp. $\ell_{2}$ ) for the label of the left (resp. right) child of the vertex with the label $\ell$. Similarly, to the proof of Theorem 6.3, there exists some $\left(\ell_{1}, \ell_{2}\right) \in J(\mathcal{T})$ that satisfies

$$
\underset{S \sim \prod_{k}^{m}}{\mathbb{E}} \underset{\left\{\mathbf{Y}_{S}\right\}}{\mathbb{E}} \underset{\left\{\mathfrak{t}_{1}, \mathfrak{t}_{2}\right\} \sim w_{\ell_{1}, \ell_{2}}}{\mathbb{E}}\left\|\left\{\mathbf{Y}_{\mathfrak{t}_{1} \sqcup \mathfrak{t}_{2}} \mid \mathbf{Y}_{S}\right\}-\left\{\mathbf{Y}_{\mathfrak{t}_{1}} \mid \mathbf{Y}_{S}\right\}\left\{\mathbf{Y}_{\mathfrak{t}_{2}} \mid \mathbf{Y}_{S}\right\}\right\|_{1} \geq \frac{\varepsilon}{2 k}
$$

Now, analogously to Eq. (15), using $\ell_{1} \leq k$ using we have

$$
\underset{\substack{S \sim \Pi_{k}^{m} \\
\left\{\mathbf{Y}_{S}\right\}}}{\mathbb{P}}\left[\underset{\mathfrak{a} \in \Pi_{\ell_{1}, \ell_{2}}}{\mathbb{E}}\left[\begin{array}{c}
\mathbb{E}_{\mathfrak{t}_{1} \in X\left(\ell_{1}\right)}\left[\operatorname{Var}\left[\mathbf{Y}_{\mathfrak{t}_{1}} \mid \mathbf{Y}_{S}\right]-\operatorname{Var}\left[\mathbf{Y}_{\mathfrak{t}_{1}} \mid \mathbf{Y}_{S}, \mathbf{Y}_{\mathfrak{a}}\right]\right] \\
+\mathbb{E}_{\mathfrak{t}_{2} \in X\left(\ell_{2}\right)}\left[\operatorname{Var}\left[\mathbf{Y}_{\mathfrak{t}_{2}}|| \mathbf{Y}_{S}\right]-\operatorname{Var}\left[\mathbf{Y}_{\mathfrak{t}_{2}} \mid \mathbf{Y}_{S}, \mathbf{Y}_{\mathfrak{a}}\right]\right]
\end{array}\right] \geq c_{5} \cdot \frac{\varepsilon^{4}}{256 \cdot k^{4} \cdot q^{4 k} \cdot r}\right] \geq \frac{\varepsilon}{4 k},
$$

Using the same arguments in the proof of Theorem 6.3, we can get that

$$
\Phi_{m}-\Phi_{m+1} \geq \frac{1}{k} \cdot \frac{\varepsilon}{4 k} \cdot \frac{c_{5} \cdot \varepsilon^{4}}{512 \cdot k^{6} \cdot q^{4 k} \cdot r}=c_{5} \cdot \frac{\varepsilon^{5}}{2048 \cdot k^{4} \cdot q^{4 k} \cdot r} .
$$

Again, this would mean that there can be at most $2048 \cdot k^{6} \cdot q^{4 k} \cdot r /\left(\varepsilon^{5} \cdot c_{5}\right)$ indices $m$ such that $\varepsilon_{m / 2} \geq \varepsilon / 2$. In particular,

$$
\underset{m \in[L / k]}{\mathbb{E}}\left[\varepsilon_{m}\right] \leq \frac{\varepsilon}{2}+\frac{k}{L} \cdot \frac{2048 \cdot k^{6} \cdot q^{4 k} \cdot r}{\varepsilon^{5} \cdot c_{5}} .
$$

i.e. there exists a universal constant $C^{\prime \prime} \geq 0$, such that

$$
L \geq C^{\prime \prime} \cdot \frac{k^{7} \cdot q^{4 k} \cdot r}{\varepsilon^{5}} \text { ensures } \underset{m \sim[L / k]}{\mathbb{E}} \varepsilon_{m} \leq \varepsilon
$$

\section{Quantum k-local Hamiltonian}

Our $k$-CSP results extend to the quantum setting generalizing the approximation scheme for 2-local Hamiltonians on bounded degree low threshold rank graphs from Brandão and Harrow [BH13] (BH). Before we can make the previous statement more precise we will need to introduce some notation. A well studied quantum analogue of classical $k$-CSPs are the so-called quantum $k$-local Hamiltonians [AAV13].

Definition 8.1 ( $k$-local Hamiltonian). We say that $\mathrm{H}=\mathbb{E}_{\mathfrak{s} \sim \Pi_{k}} \mathrm{H}_{\mathfrak{s}}$ is an instance of the $k$-local Hamiltonian problem over q-qudits on ground set $[n]$ if there is a distribution $\Pi_{k}$ on subsets of size $k$ of $[n]$ such that for every $\mathfrak{s} \in \operatorname{Supp}\left(\Pi_{k}\right)$ there is an Hermitian operator $\mathrm{H}_{\mathfrak{s}}$ on $\mathbb{C}^{q^{n}}$ with $\left\|\mathrm{H}_{\mathfrak{s}}\right\|_{\text {op }} \leq 1$ and acting (possibly) non-trivially on the q-qudits of $\mathfrak{s}$ and trivially on $[n] \backslash \mathfrak{s}$.

Given an instance $\mathrm{H}=\mathbb{E}_{\mathfrak{s} \sim \Pi_{k}} \mathrm{H}_{\mathfrak{s}}$ of the $k$-local Hamiltonian problem on ground set $[n]$, the goal is to provide a good (additive) approximation to the ground state energy $e_{0}(\mathrm{H})$, i.e., the smallest eigenvalue of $\mathrm{H}$. Equivalently, the goal is to approximate

$$
e_{0}(\mathrm{H})=\min _{\rho \in D\left(\mathbb{C}^{q^{n}}\right)} \operatorname{Tr}(\mathrm{H} \rho)
$$

where $D\left(\mathbb{C}^{q^{n}}\right)$ is the set of density operators, PSD operators of trace one, on $\mathbb{C}^{q^{n}}$. The eigenspace of $\mathrm{H}$ associated to $e_{0}(\mathrm{H})$ is called the ground space of $\mathrm{H}$. 
Remark 8.2. The locality $k$ of a $k$-local Hamiltonian has a similar role as the arity of $k$-CPSs whereas the qudit dimension $q$ has the role of alphabet size. Observe that for a $k$-CSP the goal is to maximize the fraction of satisfied constrains while for a $k$-local Hamiltonian the goal is to minimize the energy (constraint violations).

We will need an informationally complete measurement $\Lambda$ modeled as a channel

$$
\Lambda: D\left(\mathbb{C}^{q}\right) \rightarrow D\left(\mathbb{C}^{q^{8}}\right)
$$

and defined as

$$
\Lambda(\rho):=\sum_{y \in \mathcal{Y}} \operatorname{Tr}\left(\mathrm{M}_{y} \rho\right) \cdot e_{y} e_{y}^{\dagger}
$$

where $\left\{\mathrm{M}_{y}\right\}_{y \in \mathcal{Y}}$ is a $\mathrm{POVM}^{8}$ and $\left\{e_{y}\right\}_{y \in \mathcal{Y}}$ is an orthonormal basis (see Lemma 8.8 below for the properties of $\Lambda$ ). Recall that an informationally complete measurement is an injective channel, i.e., the probability outcomes $p(y)=\operatorname{Tr}\left(\mathrm{M}_{y} \rho\right)$ fully determine $\rho$. By definition given this probability distribution $\{p(y)\}_{y \in \mathcal{Y}}$ we can uniquely determine $\rho$. We use the notation $\rho=\Lambda^{-1}\left(\{p(y)\}_{y \in \mathcal{Y}}\right)$ for the recovered state from probability outcomes $\{p(y)\}_{y \in \mathcal{Y}}$.

$\mathrm{BH}$ using the informationally complete measurement $\Lambda$ reduced the quantum 2-local Hamiltonian problem to a classical problem involving PSD ensembles of indicator random variables of outcomes $\mathcal{Y}$ of $\Lambda$. In this reduction, they had to ensure that the local distributions encoded by these indicators random variables are indeed consistent with probability distributions of outcomes arising from actual local density matrices. Note that the channel $\Lambda$ is only injective, an arbitrary probability distribution on $\mathcal{Y}$ may not correspond to a valid quantum state. For this reason, they introduced a new SDP hierarchy to find this special kind of PSD ensemble, which we refer to as quantum PSD ensemble, minimizing the value of the given input $k$-local Hamiltonian instance.

Using our $k$-CSP approximation scheme for low threshold rank hypergraphs, we show that product state approximations close to the ground space of $k$-local Hamiltonians on bounded degree low threshold rank hypergraphs can be computed efficiently in polynomial time by Algorithm 8.3. Our result is a generalization of the $k=2$ case of Brandão and Harrow [BH13] for 2-local Hamiltonians on bounded degree low threshold rank graphs. Their algorithm is based on the 2-CSP result from [BRS11].

\footnotetext{
${ }^{8} \mathrm{~A}$ POVM is a collection of operators $\left\{\mathrm{M}_{y}\right\}_{y \in \mathcal{Y}}$ such that $\sum_{y \in \mathcal{Y}} M_{y}=\mathrm{I}$ and $(\forall y \in \mathcal{Y})\left(\mathrm{M}_{y} \succeq 0\right)$.
} 

Algorithm 8.3 (Quantum Propagation Rounding Algorithm).
Intput L-local quantum PSD ensemble ${ }^{a}\left\{\mathbf{Y}_{1}, \ldots, \mathbf{Y}_{n}\right\}$ and distribution $\Pi$ on $X(\leq \ell)$.
Output $A$ random state $\rho=\rho_{1} \otimes \ldots \otimes \rho_{n}$ where each $\rho_{i} \in D\left(\mathbb{C}^{q}\right)$.
1. Choose $m \in\{1, \ldots, L / \ell\}$ at random.
2. Independently sample $m \ell$-faces, $\mathfrak{s}_{j} \sim \Pi$ for $j=1, \ldots, m$.
3. Write $S=\bigcup_{j=1}^{m} \mathfrak{s}_{j}$, for the set of the seed vertices.
4. Sample assignment $\eta_{S}: S \rightarrow[q]$ according to the local distribution, $\left\{\mathbf{Y}_{S}\right\}$.
5. Set $\mathbf{Y}^{\prime}=\left\{\mathbf{Y}_{1}, \ldots \mathbf{Y}_{n} \mid \mathbf{Y}_{S}=\eta_{S}\right\}$, i.e. the local ensemble $\mathbf{Y}$ conditioned on agreeing with $\eta_{S}$.
6. For all $j \in[n]$, set $\rho_{j}=\Lambda^{-1}\left(\left\{\mathbf{Y}_{j}^{\prime}\right\}\right)$.
7. Output $\rho=\rho_{1} \otimes \ldots \otimes \rho_{n}$.

${ }^{a}$ We define the quantum ensemble as the PSD ensemble produced by the SDP hierarchy of [BH13]

The precise result is given in Theorem 8.4.

Theorem 8.4. Suppose $\mathfrak{I}=\left(\mathrm{H}=\mathbb{E}_{\mathfrak{s} \sim \Pi_{k}} \mathrm{H}_{\mathfrak{s}}\right)$ is a q-qudit $k$-local Hamiltonian instance whose constraint complex ${ }^{9}$ is $X(\leq k)$ and has bounded normalized degree, i.e., $\Pi_{1} \leq \delta$. Let $\tau=$ $c_{4} \cdot\left(\varepsilon^{2} /\left(16 k^{2} q^{8 k}\right)\right)^{2}$, for $\varepsilon>0$. There exists an absolute constant $C^{\prime}$ that satisfies the following:

Set $L=\left(\frac{C^{\prime} \cdot k^{5} \cdot q^{8 k} \cdot \operatorname{rank}_{\tau}(\mathfrak{I})}{\varepsilon^{4}}\right)$. Then there is an algorithm based on L-levels of SoS-hierarchy and Algorithm 8.3 that outputs a random product state $\rho=\rho_{1} \otimes \ldots \otimes \rho_{n}$ that in expectation ensures

$$
\operatorname{Tr}(\mathrm{H} \rho) \leq e_{0}(\mathrm{H})+(18 q)^{k / 2} \cdot \varepsilon+L \cdot k \cdot \delta,
$$

where $e_{0}(\mathrm{H})$ is the ground state energy of $\mathrm{H}$.

Remark 8.5. Similarly to the classical case, Theorem 8.4 serves as a no-go barrier (in its parameter regime) to the quantum local-Hamiltonian version of the quantum PCP Conjecture [AAV13]. In particular, $k$-local Hamiltonians on bounded degree $\gamma$-HDXs for $\gamma$ sufficiently small can be efficiently approximated in polynomial time.

Now we sketch a proof of Theorem 8.4. We provide a sketch rather than a full proof since Theorem 8.4 easily follows from the $\mathrm{BH}$ analysis once the main result used by them, Theorem 5.6 from [BRS11], is appropriately generalized to "break" $k$-wise correlations as accomplished by our Theorem 7.3 (restated below for convenience). Furthermore, a full proof would require introducing more objects and concepts only needed in this simple derivation (the reader is referred to [BH13] for the quantum terminology and the omitted details).

Theorem 8.6 (Adaptation of Theorem 7.3). Suppose a simplicial complex $X(\leq k)$ with $X(1)=$ $[n]$ and an L-local PSD ensemble $\mathbf{Y}=\left\{\mathbf{Y}_{1}, \ldots, \mathbf{Y}_{n}\right\}$ are given. There exists some universal constants $c_{4} \geq 0$ and $C^{\prime \prime} \geq 0$ satisfying the following: If $L \geq C^{\prime \prime} \cdot\left(q^{4 k} \cdot k^{7} \cdot r / \varepsilon^{5}\right)$, $\operatorname{Supp}\left(\mathbf{Y}_{j}\right) \leq q$ for all $j \in[n]$, and $\mathfrak{I}$ is $\left(c_{4} \cdot\left(\varepsilon /\left(4 k \cdot q^{8 k}\right)\right)^{4}, r\right)$-splittable. Then, we have

$$
\underset{\mathfrak{a} \in X(k)}{\mathbb{E}}\left[\left\|\left\{\mathbf{Y}_{\mathfrak{a}}^{\prime}\right\}-\left\{\mathbf{Y}_{a_{1}}^{\prime}\right\} \cdots\left\{\mathbf{Y}_{a_{k}}^{\prime}\right\}\right\|_{1}\right] \leq \varepsilon,
$$

\footnotetext{
${ }^{9}$ We define the constraint complex of a $k$-local Hamiltonian in the same way we define it for $k$-CSPs, namely, by taking the downward closure of the support of $\Pi_{k}$.
} 
where $\mathbf{Y}^{\prime}$ is as defined in Algorithm 8.3 on the input of $\left\{\mathbf{Y}_{1}, \ldots, \mathbf{Y}_{n}\right\}$ and $\Pi_{k}$.

Once in possession of the quantum PSD ensemble the problem becomes essentially classical. The key result in the $\mathrm{BH}$ approach is Theorem 5.6 from [BRS11] that brings (in expectation under conditioning on a random small seed set of qudits) the local distributions, over the edges of the constraint graph of a 2-local Hamiltonian, close to product distributions ${ }^{10}$. Now, using the fact that they have an informationally complete measurement $\Lambda$ they can "lift" the conditioned marginal distribution on each qudit $\left\{\mathbf{Y}_{j}^{\prime}\right\}$ to an actual quantum state as $\rho_{j}=\Lambda^{-1}\left(\left\{\mathbf{Y}_{j}^{\prime}\right\}\right)$ (see Algorithm 8.3). In this lifting process, they pay an average distortion cost of $18 q \cdot \varepsilon$ (for using the marginal over the qudits). For $k$-local Hamiltonians, the distortion of $k q$-qudits is given by Lemma 8.7 (stated next without proof).

Lemma 8.7. Let $\mathbf{Z}_{1}, \ldots, \mathbf{Z}_{k}$ be random variables in an $L$-local quantum PSD ensemble with $L \geq k$. Suppose that

$$
\varepsilon:=\left\|\left\{\mathbf{Z}_{1}, \ldots, \mathbf{Z}_{k}\right\}-\prod_{i=1}^{k}\left\{\mathbf{Z}_{i}\right\}\right\|_{1}
$$

Then

$$
\left\|\left(\Lambda^{\otimes k}\right)^{-1}\left(\left\{\mathbf{Z}_{1}, \ldots, \mathbf{Z}_{k}\right\}\right)-\left(\Lambda^{\otimes k}\right)^{-1}\left(\prod_{i=1}^{k}\left\{\mathbf{Z}_{i}\right\}\right)\right\|_{1} \leq(18 q)^{k / 2} \cdot \varepsilon .
$$

Note that Lemma 8.7 is a direct consequence of Lemma 8.8 from [BH13].

Lemma 8.8 (Informationally complete measurements (Lemma 16 [BH13])). For every positive integer $q$ there exists a measurement $\Lambda$ with $\leq q^{8}$ outcomes such that for every positive integer $k$ and every traceless operator $\xi$, we have

$$
\|\xi\|_{1} \leq(18 q)^{k / 2}\left\|\Lambda^{\otimes k}(\xi)\right\|_{1}
$$

$\mathrm{BH}$ also pay a full cost for each local term in the Hamiltonian that involves a seed qudit since its state was not reconstructed using the full distribution of a qudit given by the quantum ensemble but rather reconstructed from a single outcome $y \in \mathcal{Y}$ of $\Lambda$. Naively, this means that the final state of this qudit may be far from the intended state given by SDP relaxation. In our case, we assume that the normalized degree satisfies $\Pi_{1} \leq \delta$. Therefore, the total error from constraints involving seed qudits is at most

$$
L \cdot k \cdot \delta
$$

Putting the above pieces together we conclude the proof (sketch) of Theorem 8.4.

\section{Acknowledgements}

We thank Anand Louis for several enlightening discussions in the initial phases of this work. We are also grateful to the anonymous reviewers for several helpful suggestions.

\footnotetext{
${ }^{10}$ For this to hold we need the underlying constraint graph to be low threshold rank and the SoS degree to be sufficiently large
} 


\section{References}

[AAV13] Dorit Aharonov, Itai Arad, and Thomas Vidick. Guest column: The quantum PCP conjecture. SIGACT News, 44(2), 2013. 46, 48

[AJQ $\left.{ }^{+} 19\right]$ Vedat Levi Alev, Fernando Granha Jeronimo, Dylan Quintana, Shashank Srivastava, and Madhur Tulsiani. List decoding of direct sum codes. Manuscript, 2019. 4

[AKK ${ }^{+}$08] Sanjeev Arora, Subhash Khot, Alexandra Kolla, David Steurer, Madhur Tulsiani, and Nisheeth Vishnoi. Unique games on expanding constraint graphs are easy. In Proceedings of the 40th ACM Symposium on Theory of Computing, 2008. 1

[ALGV18] Nima Anari, Kuikui Liu, Shayan Oveis Gharan, and Cynthia Vinzant. Logconcave polynomials II: High-dimensional walks and an FPRAS for counting bases of a matroid. arXiv preprint arXiv:1811.01816, 2018. 4

[BH13] Fernando G. S. L. Brandão and Aram Wettroth Harrow. Product-state approximations to quantum ground states. In Proceedings of the 45th ACM Symposium on Theory of Computing, pages 871-880, 2013. 4, 46, 47, 48, 49

[BRS11] Boaz Barak, Prasad Raghavendra, and David Steurer. Rounding semidefinite programming hierarchies via global correlation. In FOCS, pages 472-481, 2011. $1,2,3,4,7,8,9,10,11,34,35,37,38,43,44,47,48,49,52,53,54,55$

[BS02] M. Bernstein and N. J. A. Sloane. Some Canonical Sequences of Integers. arXiv Mathematics e-prints, page math/0205301, May 2002. arXiv:math/0205301. 17

[BSHR05] Eli Ben-Sasson, Prahladh Harsha, and Sofya Raskhodnikova. Some 3CNF properties are hard to test. SIAM Journal on Computing, 35(1):1-21, 2005. 1

[CTZ18] David Conlon, Jonathan Tidor, and Yufei Zhao. Hypergraph expanders of all uniformities from Cayley graphs. arXiv e-prints, page arXiv:1809.06342, September 2018. arXiv:1809.06342. 2

[DD19] Yotam Dikstein and Irit Dinur. Agreement testing theorems on layered set systems. In Proceedings of the 60th IEEE Symposium on Foundations of Computer Science, 2019. 4

[DDFH18] Yotam Dikstein, Irit Dinur, Yuval Filmus, and Prahladh Harsha. Boolean function analysis on high-dimensional expanders. In Approximation, Randomization, and Combinatorial Optimization. Algorithms and Techniques, APPROX/RANDOM 2018, August 20-22, 2018 - Princeton, NJ, USA, pages 38:1$38: 20,2018.2,3,4,5,11,19,20,21,22,23,24,25,27,28,56,57$

[DHK ${ }^{+}$18] Irit Dinur, Prahladh Harsha, Tali Kaufman, Inbal Livni Navon, and Amnon Ta-Shma. List decoding with double samplers. Electronic Colloquium on Computational Complexity (ECCC), 25:136, 2018. 1, 2

[DK12] Irit Dinur and Tali Kaufman. Locally testable codes and expanders. Manuscript, 2012. 1 
[DK17] Irit Dinur and Tali Kaufman. High dimensional expanders imply agreement expanders. In Proceedings of the 58th IEEE Symposium on Foundations of Computer Science, pages 974-985, 2017. 1, 2, 3, 5, 6, 10

[Fil16] Yuval Filmus. An orthogonal basis for functions over a slice of the boolean hypercube. Electr. J. Comb., 23(1):P1.23, 2016. 32

[FK96] A. Frieze and R. Kannan. The regularity lemma and approximation schemes for dense problems. In Proceedings of the 37th IEEE Symposium on Foundations of Computer Science, 1996. 3

[GM15] Christopher Godsil and Karen Meagher. Erdős-Ko-Rado Theorems: Algebraic Approaches. Cambridge Studies in Advanced Mathematics. Cambridge University Press, 2015. 19, 32

[GS11] Venkatesan Guruswami and Ali Kemal Sinop. Lasserre hierarchy, higher eigenvalues, and approximation schemes for graph partitioning and quadratic integer programming with PSD objectives. In Proceedings of the 52nd IEEE Symposium on Foundations of Computer Science, pages 482-491, 2011. 1, 3

[GS12] Venkatesan Guruswami and Ali Kemal Sinop. Faster SDP hierarchy solvers for local rounding algorithms. In Proceedings of the 53rd IEEE Symposium on Foundations of Computer Science, pages 197-206. IEEE, 2012. 2

[Kho02] Subhash Khot. On the power of unique 2-prover 1-round games. In Proceedings of the 34th ACM Symposium on Theory of Computing, pages 767-775, 2002. 1

[KKL16] Tali Kaufman, David Kazhdan, and Alexander Lubotzky. Isoperimetric inequalities for ramanujan complexes and topological expanders. Geometric and Functional Analysis, 26(1):250-287, Feb 2016. 2

[KM17] Tali Kaufman and David Mass. High dimensional random walks and colorful expansion. In Proceedings of the 8th Conference on Innovations in Theoretical Computer Science, pages 4:1-4:27, 2017. 2, 3

[KM18] Tali Kaufman and David Mass. Good distance lattices from high dimensional expanders. CoRR, abs/1803.02849, 2018. URL: http://arxiv.org/abs/1803.02849, arXiv:1803.02849. 1

[KMOW17] Pravesh Kothari, Ryuhei Mori, Ryan O'Donnell, and David Witmer. Sum of squares lower bounds for refuting any CSP. In Proceedings of the 49th ACM Symposium on Theory of Computing, 2017. 1

[KO18a] Tali Kaufman and Izhar Oppenheim. Construction of new local spectral high dimensional expanders. In Proceedings of the 50th ACM Symposium on Theory of Computing, STOC 2018, pages 773-786. ACM, 2018. 2

[KO18b] Tali Kaufman and Izhar Oppenheim. High order random walks: Beyond spectral gap. In Approximation, Randomization, and Combinatorial Optimization. Algorithms and Techniques, APPROX/RANDOM 2018, August 20-22, 2018 - Princeton, NJ, USA, pages 47:1-47:17, 2018. 2, 3, 4, 5 
[LLP17] Eyal Lubetzky, Alex Lubotzky, and Ori Parzanchevski. Random walks on Ramanujan complexes and digraphs. arXiv e-prints, page arXiv:1702.05452, Feb 2017. arXiv:1702.05452. 3

[LSV05a] Alexander Lubotzky, Beth Samuels, and Uzi Vishne. Explicit constructions of ramanujan complexes of type ad. Eur. J. Comb., 26(6):965-993, August 2005. 2

[LSV05b] Alexander Lubotzky, Beth Samuels, and Uzi Vishne. Ramanujan complexes of typeãd. Israel Journal of Mathematics, 149(1):267-299, Dec 2005. 2

[MM11] Konstantin Makarychev and Yury Makarychev. How to play unique games on expanders. In Approximation and Online Algorithms, pages 190-200. Springer Berlin Heidelberg, 2011. 1

[MR17] Pasin Manurangsi and Prasad Raghavendra. A birthday repetition theorem and complexity of approximating dense csps. In Proceedings of the 44th International Colloquium on Automata, Languages and Programming. Schloss DagstuhlLeibniz-Zentrum fuer Informatik, 2017. 4

[OGT15] Shayan Oveis Gharan and Luca Trevisan. A new regularity lemma and faster approximation algorithms for low threshold rank graphs. Theory of Computing, 11(9):241-256, 2015. URL: http://www.theoryof computing.org/articles/v011a009, doi:10.4086/toc.2015.v011a009.1,3

[PRT16] Ori Parzanchevski, Ron Rosenthal, and Ran J. Tessler. Isoperimetric inequalities in simplicial complexes. Combinatorica, 36(2):195-227, Apr 2016. 2

[RW17] Prasad Raghavendra and Benjamin Weitz. On the bit complexity of sumof-squares proofs. In Proceedings of the 44th International Colloquium on Automata, Languages and Programming. Schloss Dagstuhl-Leibniz-Zentrum fuer Informatik, 2017. 2

[Sag13] B.E. Sagan. The Symmetric Group: Representations, Combinatorial Algorithms, and Symmetric Functions. Graduate Texts in Mathematics. Springer New York, 2013. 32

[WJ04] Martin J Wainwright and Michael I Jordan. Treewidth-based conditions for exactness of the Sherali-Adams and Lasserre relaxations. Technical report, Technical Report 671, University of California, Berkeley, 2004. 4

[YZ14] Yuichi Yoshida and Yuan Zhou. Approximation schemes via Sherali-Adams hierarchy for dense constraint satisfaction problems and assignment problems. In Proceedings of the 5th Conference on Innovations in Theoretical Computer Science, pages 423-438. ACM, 2014. 4

\section{A From Local to Global Correlation}

We include the key result we use from [BRS11], namely, their Lemma 5.4 (below). While they proved the lemma for regular graphs, we include the details in the proof for general 
weighted graphs, since even for HDXs regular at the top level, the swap graphs are not necesarily regular. The extension to general graphs is straighforward (and [BRS11] indicated the same) but we include the details for the sake of completeness ${ }^{11}$.

Lemma A.1 (Lemma 5.4 from [BRS11] (restatement of Lemma 7.5)). Let $G=\left(V, E, \Pi_{2}\right)$ be a weighted graph, $\left\{\mathbf{Y}_{1}, \ldots, \mathbf{Y}_{n}\right\}$ a local PSD ensemble, where we have $\operatorname{Supp}\left(\mathbf{Y}_{i}\right) \leq q$ for every $i \in V$, and $q \geq 0$. If $\varepsilon \geq 0$ is a lower bound on the expected statistical difference between independent and correlated sampling along the edges,i.e.,

$$
\varepsilon \leq \underset{\{i, j\} \sim \Pi_{2}}{\mathbb{E}}\left[\left\|\left\{\mathbf{Y}_{i j}\right\}-\left\{\mathbf{Y}_{i}\right\}\left\{\mathbf{Y}_{j}\right\}\right\|_{1}\right]
$$

Then, conditioning on a random vertex decreases the variances,

$$
\underset{i, j \sim \Pi_{1}}{\mathbb{E}}\left[\underset{\left\{\mathbf{Y}_{j}\right\}}{\mathbb{E}}\left[\operatorname{Var}\left[\mathbf{Y}_{i} \mid \mathbf{Y}_{j}\right]\right]\right] \leq \underset{i \sim \Pi_{1}}{\mathbb{E}}\left[\operatorname{Var}\left[\mathbf{Y}_{i}\right]\right]-\frac{\varepsilon^{4}}{4 q^{4} \cdot \operatorname{rank}_{\varepsilon^{2} /\left(4 q^{2}\right)}(G)}
$$

The key ingredient in proving Lemma 5.4 is a "local to global" argument generalizing the expander case to low threshold rank graphs. This new argument is proven in two steps with Lemma A.2 being the first one.

Lemma A.2 (Adapted from Lemma 6.1 of [BRS11]). Let $G$ be an undirected weighted graph. Suppose $v_{1}, \ldots, v_{n} \in \mathbb{R}^{n}$ are such that

$$
\underset{i \sim V(G)}{\mathbb{E}}\left[\left\langle v_{i}, v_{i}\right\rangle\right]=1, \quad \underset{i j \sim E(G)}{\mathbb{E}}\left[\left\langle v_{i}, v_{j}\right\rangle\right] \geq 1-\varepsilon,
$$

but

$$
\underset{i, j \sim V(G)}{\mathbb{E}}\left[\left\langle v_{i}, v_{j}\right\rangle^{2}\right] \leq \frac{1}{m}
$$

Then for $c>1$, we have

$$
\lambda_{\left(1-\frac{1}{c}\right)^{2} m} \geq 1-c \cdot \varepsilon
$$

In particular, $\lambda_{m / 4} \geq 1-2 \varepsilon$.

Proof. Let $\mathrm{Y}$ be the Gram matrix defined as $\mathrm{Y}_{i, j}=\left\langle v_{i}, v_{j}\right\rangle$. Clearly, $\mathrm{Y}$ is positive semidefinite. Without loss of generality suppose that the edge weights $\{w(\{i, j\}) \mid i j \in E(G)\}$ form a probability distribution. Set $w(i)=\sum_{j \sim i} w(\{i, j\})$. Let $\mathrm{D}$ to be the diagonal matrix such that $\mathrm{D}(i, i)=w(i)$, i.e., the matrix of generalized degrees. Let $\mathrm{A}$ be such that $\mathrm{A}_{i, j}=$ $w(\{i, j\}) / 2$ and $A_{G}=D^{-1 / 2} A D^{-1 / 2}$ be its normalized adjacency matrix.

Suppose $\mathrm{A}_{G}=\sum_{i=1}^{n} \lambda_{i} u_{i} u_{i}^{\top}$ is a spectral decomposition of $\mathrm{A}_{G}$. Set $\mathrm{Y}^{\prime}=\mathrm{D}^{1 / 2} \mathrm{YD}^{1 / 2}$. For convenience, define the matrix $\mathrm{X}$ as $\mathrm{X}(i, j)=\left\langle u_{i}, \mathrm{Y}^{\prime} u_{j}\right\rangle$ and set $p(i)=\mathrm{X}(i, i)$. We claim that $p$ is a probability distribution. Since $Y^{\prime}$ is positive semi-definite, we have that $p(i) \geq 0$. Moreover, $\sum_{i=1}^{n} p(i)=1$ as

$$
1=\underset{i \sim V(G)}{\mathbb{E}}\left[\left\langle v_{i}, v_{i}\right\rangle\right]=\operatorname{Tr}\left(\mathrm{Y}^{\prime}\right)=\operatorname{Tr}(\mathrm{X})=\sum_{i=1}^{n} \mathrm{X}(i, i)=\sum_{i=1}^{n} p(i) .
$$

\footnotetext{
${ }^{11}$ For expander graphs it is possible to obtain an improved bound of $\Omega\left((\varepsilon / q)^{2}\right)$ instead of $\Omega\left((\varepsilon / q)^{4}\right)$ given by Lemma A.1, simply by using the definition of the second smallest eigenvalue of the Laplacian. While BRS analyzed $\mathbb{E}_{i, j}\left[\left\langle v_{i}, v_{j}\right\rangle^{2}\right]$ for low-threshold rank graphs, it is possible to directly analyze the quantity $\mathbb{E}_{i, j}\left[\left\langle v_{i}, v_{j}\right\rangle\right]$ for expanders, leading to the improved bound.
} 
Let $m^{\prime}$ be the largest value in $[n]$ satisfying $\lambda_{m^{\prime}} \geq 1-c \cdot \varepsilon$. By Cauchy-Schwarz ${ }^{12}$,

$$
q=\sum_{i=1}^{m^{\prime}} p(i) \leq \sqrt{m^{\prime}} \sqrt{\sum_{i=1}^{m^{\prime}} p(i)^{2}} \leq \sqrt{m^{\prime}} \sqrt{\sum_{i, j}(\mathbf{X}(i, j))^{2}} \leq \sqrt{\frac{m^{\prime}}{m}},
$$

where the last inequality follows from our assumption that

$$
\frac{1}{m} \geq \underset{i, j \sim V(G)}{\mathbb{E}}\left[\left\langle v_{i}, v_{j}\right\rangle^{2}\right]=\left\langle\mathrm{Y}^{\prime}, \mathrm{Y}^{\prime}\right\rangle=\langle\mathrm{X}, \mathrm{X}\rangle=\sum_{i, j} \mathrm{X}(i, j)^{2}
$$

Then

$$
1-\varepsilon \leq \underset{i j \sim E(G)}{\mathbb{E}}\left[\left\langle v_{i}, v_{j}\right\rangle\right]=\langle\mathrm{A}, \mathrm{Y}\rangle=\left\langle\mathrm{A}_{G}, \mathrm{X}\right\rangle=\sum_{i=1}^{n} \lambda_{i} \mathrm{X}(i, i),
$$

implies that

$$
1-\varepsilon \leq \sum_{i=1}^{n} \lambda_{i} \cdot \mathbf{X}(i, i) \leq \sum_{i=1}^{m^{\prime}} p(i)+(1-c \cdot \varepsilon) \sum_{i=m^{\prime}+1}^{n} p(i)=1-c \cdot \varepsilon(1-q) .
$$

Finally, using the bound on $q$ we obtain

$$
\left(1-\frac{1}{c}\right) \sqrt{m} \leq \sqrt{m^{\prime}}
$$

from which the lemma readily follows.

As a corollary it follows that local correlation implies global correlation.

Corollary A.3 (Adapted from Lemma 4.1 of [BRS11]). Let $G$ be an undirected weighted graph. Suppose $v_{1}, \ldots, v_{n} \in \mathbb{R}^{n}$ are vectors in the unit ball such that

$$
\underset{i j \sim E(G)}{\mathbb{E}}\left[\left\langle v_{i}, v_{j}\right\rangle\right] \geq \rho,
$$

then

$$
\underset{i, j \sim V(G)}{\mathbb{E}}\left[\left\langle v_{i}, v_{j}\right\rangle^{2}\right] \geq \frac{\rho^{2}}{4 \cdot \operatorname{rank}_{\rho / 4}(G)}
$$

In particular, we have

$$
\underset{i, j \sim V(G)}{\mathbb{E}}\left[\left|\left\langle v_{i}, v_{j}\right\rangle\right|\right] \geq \frac{\rho^{2}}{4 \cdot \operatorname{rank}_{\rho / 4}(G)}
$$

Proof. If all $v_{1}, \ldots, v_{n}$ are zero, the result trivially follows so assume that this is not the case. Then $\alpha=\mathbb{E}_{i \sim V(G)}\left[\left\langle v_{i}, v_{i}\right\rangle\right]>0$. Also, $\alpha \leq 1$ since the vectors lie in the unit ball. Let $v_{i}^{\prime}=v_{i} / \sqrt{\alpha}$. By construction

$$
\underset{i \sim V(G)}{\mathbb{E}}\left[\left\langle v_{i}^{\prime}, v_{i}^{\prime}\right\rangle\right]=1, \quad \underset{i j \sim E(G)}{\mathbb{E}}\left[\left\langle v_{i}^{\prime}, v_{j}^{\prime}\right\rangle\right] \geq \frac{\rho}{\alpha}
$$

\footnotetext{
${ }^{12}$ In [BRS11], there was a minor bug in the application this Cauchy-Schwarz, which led to a bound of $(1-$ $1 / c)$ instead of $(1-1 / c)^{2}$ in the lemma, leading to a global correlation bound of $\Omega(\rho)$ instead of $\Omega\left(\rho^{2}\right)$ as indicated in Corollary A.3.
} 
Under these assumptions we want to apply Lemma A.2 in the contra-positive, but first we set some parameters. Let $\rho^{\prime}=\rho /(2 \alpha), \varepsilon=1-\rho^{\prime}$ and $c=\left(1-\rho^{\prime} / 2\right) /\left(1-\rho^{\prime}\right)$. Then

$$
1-\frac{1}{c}=\frac{\rho^{\prime} / 2}{1-\rho^{\prime} / 2} \leq \rho^{\prime}
$$

and

$$
1-c \cdot \varepsilon=\frac{\rho^{\prime}}{2}
$$

Now, considering the contra-positive of the Lemma A.2 under the Eq. (20) we obtain

$$
\underset{i, j \sim V(G)}{\mathbb{E}}\left[\left\langle v_{i}^{\prime}, v_{j}^{\prime}\right\rangle^{2}\right]>\frac{1}{m} \geq \frac{\left(\rho^{\prime}\right)^{2}}{\operatorname{rank}_{\rho^{\prime} / 2}(G)},
$$

since $\operatorname{rank}_{\rho^{\prime} / 2}(G)<\left(\rho^{\prime}\right)^{2} m$ as $\lambda_{\left(\rho^{\prime}\right)^{2} m}<\rho^{\prime} / 2$. Or equivalently

$$
\underset{i, j \sim V(G)}{\mathbb{E}}\left[\frac{\left\langle v_{i}, v_{j}\right\rangle^{2}}{\alpha^{2}}\right]=\underset{i, j \sim V(G)}{\mathbb{E}}\left[\left\langle v_{i}^{\prime}, v_{j}^{\prime}\right\rangle^{2}\right] \geq \frac{\rho^{2}}{4 \alpha^{2} \cdot \operatorname{rank}_{\rho /(4 \alpha)}(G)} \geq \frac{\rho^{2}}{4 \alpha^{2} \cdot \operatorname{rank}_{\rho / 4}(G)},
$$

where the last inequality follows form the fact that $\alpha \leq 1$.

To finish the proof of Lemma 5.4, we state the following Fact A.4 extracted from [BRS11].

Fact A.4 (Adapted from [BRS11]). Let $\left\{\mathbf{Y}_{1}, \ldots, \mathbf{Y}_{n}\right\}$ be a 2-local PSD ensemble where each $\mathbf{Y}_{i}$ can take at most $q$ values. Suppose

$$
\varepsilon=\underset{\{i, j\} \sim \Pi_{2}}{\mathbb{E}}\left[\left\|\left\{\mathbf{Y}_{i j}\right\}-\left\{\mathbf{Y}_{i}\right\}\left\{\mathbf{Y}_{j}\right\}\right\|_{1}\right]
$$

Then there exist vectors $v_{1}, \ldots, v_{n}$ in the unit ball such that

$$
\underset{i j \sim E(G)}{\mathbb{E}}\left[\left\langle v_{i}, v_{j}\right\rangle\right] \geq \frac{1}{q^{2}} \cdot \underset{i j \sim \Pi_{2}}{\mathbb{E}}\left[\left\|\left\{\mathbf{Y}_{i j}\right\}-\left\{\mathbf{Y}_{i}\right\}\left\{\mathbf{Y}_{j}\right\}\right\|_{1}^{2}\right] \geq \frac{\varepsilon^{2}}{q^{2}}
$$

and

$$
\underset{i, j \sim V(G)}{\mathbb{E}}\left[\operatorname{Var}\left[\mathbf{Y}_{i}\right]-\underset{\left\{\mathbf{Y}_{j}\right\}}{\mathbb{E}}\left[\operatorname{Var}\left[\mathbf{Y}_{i} \mid \mathbf{Y}_{j}\right]\right]\right] \geq \underset{i, j \sim V(G)}{\mathbb{E}}\left[\left|\left\langle v_{i}, v_{j}\right\rangle\right|\right] .
$$

Now we are ready to prove the key result from [BRS11] used in our proof.

Lemma A.5 (Lemma 5.4 from [BRS11] (restatement of Lemma 7.5)). Let $G=\left(V, E, \Pi_{2}\right)$ be a weighted graph, $\left\{\mathbf{Y}_{1}, \ldots, \mathbf{Y}_{n}\right\}$ a local PSD ensemble, where we have $\operatorname{Supp}\left(\mathbf{Y}_{i}\right) \leq q$ for every $i \in V$, and $q \geq 0$. If $\varepsilon \geq 0$ is a lower bound on the expected statistical difference between independent and correlated sampling along the edges,i.e.,

$$
\varepsilon \leq \underset{\{i, j\} \sim \Pi_{2}}{\mathbb{E}}\left[\left\|\left\{\mathbf{Y}_{i j}\right\}-\left\{\mathbf{Y}_{i}\right\}\left\{\mathbf{Y}_{j}\right\}\right\|_{1}\right]
$$

Then, conditioning on a random vertex decreases the variances,

$$
\underset{i, j \sim \Pi_{1}}{\mathbb{E}}\left[\underset{\left\{\mathbf{Y}_{j}\right\}}{\mathbb{E}}\left[\operatorname{Var}\left[\mathbf{Y}_{i} \mid \mathbf{Y}_{j}\right]\right]\right] \leq \underset{i \sim \Pi_{1}}{\mathbb{E}}\left[\operatorname{Var}\left[\mathbf{Y}_{i}\right]\right]-\frac{\varepsilon^{4}}{4 q^{4} \cdot \operatorname{rank}_{\varepsilon^{2} /\left(4 q^{2}\right)}(G)}
$$


Proof. Using Eq. (21) there exist vectors $v_{1}, \ldots, v_{n}$ such that Fact A.4 implies

$$
\underset{i j \sim E(G)}{\mathbb{E}}\left[\left\langle v_{i}, v_{j}\right\rangle\right] \geq \frac{\varepsilon^{2}}{q^{2}}
$$

From Corollary A.3 we obtain

$$
\underset{i, j \sim V(G)}{\mathbb{E}}\left[\left|\left\langle v_{i}, v_{j}\right\rangle\right|\right] \geq \frac{\varepsilon^{4}}{4 q^{4} \cdot \operatorname{rank}_{\varepsilon^{2} /\left(4 q^{2}\right)}(G)} .
$$

Finally, using Eq. (22) we get

$$
\underset{i, j \sim V(G)}{\mathbb{E}}\left[\operatorname{Var}\left[\mathbf{Y}_{i}\right]-\underset{\left\{\mathbf{Y}_{j}\right\}}{\mathbb{E}}\left[\operatorname{Var}\left[\mathbf{Y}_{i} \mid \mathbf{Y}_{j}\right]\right]\right] \geq \underset{i, j \sim V(G)}{\mathbb{E}}\left[\left|\left\langle v_{i}, v_{j}\right\rangle\right|\right] \geq \frac{\varepsilon^{4}}{4 q^{4} \cdot \operatorname{rank}_{\varepsilon^{2} /\left(4 q^{2}\right)}(G)},
$$

as claimed.

\section{B Harmonic Analysis on HDXs}

We provide the proofs of known facts used in Section 5.2.

Definition B.1 (From [DDFH18]). We say that d-sized complex $X$ is proper provided $\operatorname{ker}\left(\mathrm{U}_{i}\right)$ is trivial for $1 \leq i<d$.

We will need the following decomposition.

Claim B.2. Let $\mathrm{A}: V \rightarrow W$ where $V$ and $W$ are finite dimensional inner product spaces. Then

$$
V=\operatorname{ker} \mathrm{A} \oplus \operatorname{im} \mathrm{A}^{\dagger}
$$

Proof. We show that ker $\mathrm{A}=\left(\operatorname{im~} \mathrm{A}^{+}\right)^{\perp}$. Recall that $v \in\left(\mathrm{im}^{+}\right)^{\perp}$ if and only if $\left\langle\mathrm{A}^{\dagger} w, v\right\rangle=0$ for every $w \in W$. This is equivalent to $\langle w, A v\rangle=0$ for every $w \in W$, implying $A v=0$.

Lemma B.3 (From [DDFH18]). We have

$$
C^{k}=\sum_{i=0}^{k} C_{i}^{k}
$$

Moreover, if $X$ is proper then

$$
C^{k}=\bigoplus_{i=0}^{k} C_{i}^{k}
$$

and $\operatorname{dim} C_{i}^{k}=|X(i)|-|X(i-1)|$.

Proof. We induct on $k$. For $k=0, X(0)=\{\varnothing\}$ and $C^{0}=C_{0}^{0}$. Now suppose $k>0$. Since $D_{k}$ and $\mathrm{U}_{k-1}$ are adjoints, we have $C^{k}=\operatorname{ker} \mathrm{D}_{k} \oplus \mathrm{im} \mathrm{U}_{k-1}$ or equivalently

$$
C^{k}=\operatorname{ker} \mathrm{D}_{k} \oplus \mathrm{U}_{k-1} C^{k-1} .
$$


Using the induction hypothesis $C^{k-1}=\sum_{i=0}^{k-1} C_{i}^{k-1}$. Note that

$$
\mathrm{U}_{k-1} C_{i}^{k-1}=\left\{\mathrm{U}_{k-1} \mathrm{U}^{k-1-i} h_{i} \mid h_{i} \in H_{i}\right\}=C_{i}^{k} .
$$

Thus $C^{k}=C_{k}^{k}+\sum_{i=0}^{k-1} C_{i}^{k}$. Assuming $\operatorname{ker}\left(\mathrm{U}_{i}\right)$ is trivial for $0 \leq i<k$ we obtain

$$
\operatorname{dim} C_{i}^{k}=\operatorname{dim} H_{i}=\operatorname{dim} C^{i}-\operatorname{dim} C^{i-1}=|X(i)|-|X(i-1)|,
$$

where the second equality follows from Eq. (23). Hence $\operatorname{dim} C^{k}=\sum_{i=0}^{k} \operatorname{dim} C_{i}^{k}$. This implies that each $C_{i}^{k} \cap \sum_{j \neq i} C_{j}^{k}$ is trivial and so we have a direct sum as claimed.

Corollary B.4 (From [DDFH18]). Let $f \in C^{k}$. If $X$ is proper, then $f$ can be written uniquely as

$$
f=f_{0}+\cdots+f_{k},
$$

where $f_{i} \in C_{i}^{k}$. 